\title{
Hydrodynamic representation of the Klein-Gordon-Einstein equations in the weak field limit: I. General formalism and perturbations analysis
}

\author{
Abril Suárez ${ }^{1}$ and Pierre-Henri Chavanis ${ }^{1}$ \\ ${ }^{1}$ Laboratoire de Physique Théorique, Université Paul Sabatier, 118 route de Narbonne 31062 Toulouse, France
}

\begin{abstract}
Using a generalization of the Madelung transformation, we derive the hydrodynamic representation of the Klein-Gordon-Einstein equations in the weak field limit. We consider a complex selfinteracting scalar field with a $\lambda|\varphi|^{4}$ potential. We study the evolution of the spatially homogeneous background in the fluid representation and derive the linearized equations describing the evolution of small perturbations in a static and in an expanding universe. We compare the results with simplified models in which the gravitational potential is introduced by hand in the Klein-Gordon equation, and assumed to satisfy a (generalized) Poisson equation. Nonrelativistic hydrodynamic equations based on the Schrödinger-Poisson equations or on the Gross-Pitaevskii-Poisson equations are recovered in the limit $c \rightarrow+\infty$. We study the evolution of the perturbations in the matter era using the nonrelativistic limit of our formalism. Perturbations whose wavelength is below the Jeans length oscillate in time while perturbations whose wavelength is above the Jeans length grow linearly with the scale factor as in the cold dark matter model. The growth of perturbations in the scalar field model is substantially faster than in the cold dark matter model. When the wavelength of the perturbations approaches the cosmological horizon (Hubble length), a relativistic treatment is mandatory. In that case, we find that relativistic effects attenuate or even prevent the growth of perturbations. This paper exposes the general formalism and provides illustrations in simple cases. Other applications of our formalism will be considered in companion papers.
\end{abstract}

PACS numbers: 95.35.+d, 98.80.-k, 98.80.Jk, 04.40.-b, 95.30.Sf

\section{INTRODUCTION}

Scalar fields (SF) play an important role in particle physics, astrophysics, and cosmology [1 $1+3$. Their evolution is usually described by the Klein-Gordon (KG) equation [4-6] which can be viewed as a relativistic extension of the Schrödinger equation [7, 8]. The KG equation was one of the first attempts to unify the ideas of quantum mechanics and Einstein's theory of special relativity. ${ }^{1}$ It describes the evolution of a SF $\varphi(\vec{x}, t)$ whose excitations are bosonic particles of spin zero. These particles are neutral for a real SF and charged for a complex SF. The KG equation usually involves a potential $V(\varphi)$ that takes selfinteraction into account. For example, $\pi$-mesons, Higgs bosons and axions are described by the KG equation. SF are also present in the theory of superstrings as well as in a large number of Kaluza-Klein and supergravity models.

The coupling between the KG equation and gravity through the Einstein equations, leading to the KleinGordon-Einstein (KGE) equations, was first considered in the context of boson stars $\left[932 .{ }^{2}\right.$ Initially, the study of boson stars was motivated by the axion field, a pseudoNambu-Goldstone boson of the Peccei-Quinn phase transition, that was proposed as a possible solution to the

\footnotetext{
1 The KG equation was actually discovered by Schrödinger before he found the equation that now bears his name [2].

2 Boson stars are described by complex SFs. Self-gravitating systems described by real SFs are not static but, instead, are periodic with both the spacetime geometry and the matter field oscillating in time. For that reason they are called oscillatons 3334.
}

strong CP problem in QCD. In the early works of Kaup [9] and Ruffini and Bonazzola [10, it was assumed that the bosons have no self-interaction. For a boson mass $m \sim 1 \mathrm{GeV} / c^{2}$, the maximum mass of boson stars is very small, of the order of $M_{\max } \sim 10^{-19} M_{\odot}$. This corresponds to mini boson stars like axion black holes. The mass of these mini boson stars may be too small to be astrophysically relevant. They could play a role, however, if they exist in the universe in abundance or if the axion mass is extraordinarily small (less than $m \sim 10^{-10} \mathrm{eV} / c^{2}$ ) leading to macroscopic objects with a mass $M_{\max }$ comparable to the mass of the sun (or even larger) [30. It has also been proposed that stable boson stars with a boson mass $m \sim 10^{-17} \mathrm{eV} / c^{2}$ could mimic supermassive black holes $\left(M \sim 10^{6} M_{\odot}, R \sim 10^{7} \mathrm{~km}\right)$ that reside at the center of galaxies [28, 31]. On the other hand, Colpi et al. [14] considered the case where the bosons have a repulsive self-interaction described by a $\lambda|\varphi|^{4}$ potential and showed that, for $m \sim 1 \mathrm{GeV} / c^{2}$, the maximum mass of boson stars can be of the order of the solar mass $M_{\odot}$, similar to the mass of neutron stars. Therefore, a self-interaction can significantly change the physical dimensions of boson stars, making them much more astrophysically interesting.

It has also been proposed that dark matter (DM) halos may be made of a SF described by the KGE equations (see, e.g., 35 37] for recent reviews). Actually, at the galactic scale, the Newtonian limit is valid so DM halos can be described by the Schrödinger-Poisson (SP) equations or by the Gross-Pitaevskii-Poisson (GPP) equations. In that case, the wavefunction $\psi(\vec{x}, t)$ describes a Bose-Einstein condensate (BEC) at $T=0$, and the self-interaction of the bosons is measured by their scat- 
tering length $a_{s}$. Therefore, DM halos could be gigantic quantum objects made of BECs. The wave properties of bosonic DM may stabilize the system against gravitational collapse, providing halo cores and sharply suppressing small-scale linear power. This may solve the problems of the cold dark matter (CDM) model such as the cusp problem and the missing satellite problem. The scalar field dark matter (SFDM) model and the BEC dark matter (BECDM) model, also called $\Psi \mathrm{DM}$ models, have received much attention in the last years. In the non-interacting case [38 61, the mass of the bosons must be extremely small, of the order of $m \sim$ $2.57 \times 10^{-20} \mathrm{eV} / c^{2}$, in order to account for the mass and size of dwarf DM halos that are completely condensed. Ultralight scalar fields like axions may have such small masses. This corresponds to "fuzzy cold dark matter" [45. On the other hand, when a repulsive self-interaction is taken into account [57, 58, 62, 75], the characteristics of dwarf DM halos can be reproduced with a much larger boson mass $m=1.69 \times 10^{-2} \mathrm{eV} / c^{2}$ (satisfying the limit $m<1.87 \mathrm{eV} / c^{2}$ obtained from cosmological considerations [76]) and a scattering length $a_{s}=1.73 \times 10^{-5} \mathrm{fm}$ (satisfying the constraint $4 \pi a_{s}^{2} / m<1.25 \mathrm{~cm}^{2} / \mathrm{g}$ set by the Bullet Cluster [77]). Dwarf halos are purely condensed. Large dark matter halos have a core-halo structure resulting from gravitational cooling [24]. They are made of a solitonic core (BEC) surrounded by a halo of scalar radiation.

Scalar fields have also been introduced in cosmology. The phase of inflation in the early universe is usually described by some hypothetical SF, called the inflaton, with its origin in the quantum fluctuations of the vacuum 78 82. A variety of inflationary models that include SFs have been proposed [83, and SF are expected to play an important role in determining the dynamics of the early universe. Inflation is generally considered to be a reasonable solution to many of the fundamental problems within the standard cosmological model. Inspired by the analogy with the inflation, some authors have represented the dark energy responsible for the present acceleration of the Universe by a SF called quintessence [84 96. This treatment seems to fit current observations, and unlike the cosmological constant $\Lambda$, the SF evolves dynamically, leaving distinctive imprints in the Cosmic Microwave Background (CMB) and matter power spectrum.

Since DM may be a SF, it is of considerable interest to study the cosmological implications of this scenario. The cosmological evolution of a spatially homogeneous noninteracting real SF described by the KGE equations competing with baryonic matter, radiation and dark energy, was considered by Matos et al. [53. They found that real SFs display fast oscillations but that, on the mean, they reproduce the cosmological predictions of the standard $\Lambda$ CDM model. The study of perturbations was considered by Suárez and Matos [97] for a self-interacting real SF described by the Klein-Gordon-Poisson (KGP) equations and by Magaña et al. 98] for a non-interacting real SF described by the KGE equations. These studies show that the perturbations can grow in the linear regime, leading, in the nonlinear regime, to the formation of structures corresponding to DM halos. This is in agreement with the early work of Khlopov et al. 99. who studied the Jeans instability of a relativistic SF in a static background.

The case of a complex self-interacting SF representing BECDM was considered by Chavanis [57, 100] in the context of Newtonian cosmology. His study is based on the GPP equations. The Jeans instability of a homogeneous self-gravitating BEC in a static background is studied in [57. The evolution of perturbations of BECDM in an expanding Einstein-deSitter (EdS) universe is considered in [100]. It is found that the perturbations grow faster in BECDM as compared to $\Lambda$ CDM. Harko 101] and Chavanis [100] independently developed a relativistic BEC cosmology by assuming that the equation of state of BECDM is given by $P=2 \pi a_{s} \hbar^{2} \epsilon^{2} / m^{3} c^{4}$, which corresponds to the classical equation of state of BECs [102] where $\rho$ is replaced by $\epsilon / c^{2}$. However, the identification of the energy density $\epsilon$ with the rest-mass density $\rho$ is only valid in a weakly relativistic regime, so the extrapolation of their results to early times is not correct (see the discussion in [103]). Recently, Li et al. [104] developed an exact relativistic cosmology for a complex self-interacting $\mathrm{SF} / \mathrm{BEC}$ based on the KGE equations. They studied the evolution of the homogeneous background and showed that the Universe undergoes three successive phases: a stiff matter era, followed by a radiation era due to the SF (that exists only for self-interacting SFs), and finally a matter era similar to CDM. They compared their theoretical results with observations in order to constrain the parameters $(m, \lambda)$ of the SF.

Instead of working directly in terms of a SF, we can adopt a fluid approach and work with hydrodynamic equations. In the case of the Schrödinger equation, this hydrodynamic approach was introduced by Madelung 105. He showed that the Schrödinger equation is equivalent to the Euler equations for an irrotational fluid with an additional quantum potential ${ }^{3}$ arising from the finite value of $\hbar$. This hydrodynamic formulation was criticized, or disregarded, by many authors (e.g., Pauli) in the early years of quantum mechanics because it lacks a clear physical interpretation in the case where the Schrödinger equation describes just one particle. However, it takes more sense when the Schrödinger equation (or the GP equation) describes a BEC made of many particles in the same quantum state 102. In that case, the BEC can be interpreted as a real fluid described by quantum

\footnotetext{
3 The results of Madelung [105] were rediscovered by Bohm [106] so the quantum potential is sometimes called the Bohm potential. The formulation of classical and relativistic quantum mechanics in terms of hydrodynamic equations was also developed by Takabayasi 107. 108.
} 
Euler equations. ${ }^{4}$ This hydrodynamic representation has been used by Böhmer and Harko 67 and by Chavanis [57, 58, 100] among others in the case of BECDM and in the case of BEC stars 32. This hydrodynamic approach has been generalized by Suárez and Matos [97] in the context of the KG equation. They used it to study the formation of structures in the Universe, assuming that $\mathrm{DM}$ is in the form of a fundamental SF with a $\lambda \varphi^{4}$ potential 97. They also studied the phase transition of a real SF due to a $Z_{2}$ symmetry of its potential [111.

In the works [97, 111], the SF is taken to be real and the gravitational potential is introduced by hand in the KG equation, and assumed to be determined by the classical Poisson equation where the source is the rest-mass density $\rho$. This leads to the KGP equations. However, this treatment is not self-consistent since it combines relativistic and nonrelativistic equations. In this article, we derive the hydrodynamic representation of a complex $\mathrm{SF}$ coupled to gravity through the Einstein equations in the weak field approximation. In this way, we develop a self-consistent relativistic treatment. Throughout this work, we use the Newtonian gauge which takes into account metric perturbations up to first order. We consider only scalar perturbations. This is sufficient if we are interested in calculating observational consequences of the $\mathrm{SF}$ dynamics in the linear regime. After having derived the hydrodynamic equations, we study the evolution of the homogeneous background and the evolution of small perturbations in a static and in an expanding universe using the hydrodynamic representation. We compare our results with those obtained from the heuristic KGP equations. We argue that this simplified model is not sufficient to study the evolution of the perturbations in the linear relativistic regime.

The main purpose of the present paper is to develop a general formalism. Applications of this formalism will be considered in following papers. However, for illustration, we already present a few applications of our formalism in simple cases.

The paper is organized as follows. In Sec. II we present the theoretical background and introduce the main equations of the the paper that are the KG equation and the Einstein field equations. This allows us to set the notations. In Sec. III we consider the KGE equations in the weak field limit. We first write the KG equation and the Einstein equations in the Newtonian gauge. Then, we transform the KGE equations into the Gross-Pitaevskii-Einstein (GPE) equations to facilitate the connection with the nonrelativistic limit $c \rightarrow+\infty$. Finally, we write the GPE equations in the form of hydrodynamic equations. In Sec. IV] we consider the case of a static background and study the evolution of small

\footnotetext{
${ }^{4}$ One interesting aspects of BECs is related to their superfluid properties. Their velocity field is irrotational but there may exist vortical motion due to singular point vortices with quantized circulation $h / m$ 109, 110.
}

perturbations in an infinite homogeneous Universe (Jeans problem). We determine the energy of the homogeneous $\mathrm{SF}$, the dispersion relation of the perturbations, and the Jeans length. In Sec. V] we consider the evolution of the Universe induced by a homogeneous SF. We recover from the fluid equations the three phases previously obtained by Li et al. [104]: a stiff matter phase, a radiation phase, and a matter phase. In Sec. VI] we consider the evolution of the perturbations in an expanding homogeneous Universe. We provide the exact set of linearized equations, then propose a closed approximate equation for the density contrast that has the correct Jeans length. In Sec. VII] we study the evolution of the perturbations in the matter era. We show that the nonrelativistic limit can be used when the wavelength of the perturbations is much smaller than the cosmological horizon (Hubble length). Perturbations whose wavelength is below the Jeans length oscillate in time while perturbations whose wavelength is above the Jeans length grow linearly with the scale factor as in the CDM model. The growth of perturbations in the scalar field model is substantially faster than in the CDM model. When the wavelength of the perturbations approaches the cosmological horizon, general relativity attenuates or even prevents the growth of perturbations. The Appendices present useful complements. In Appendix A, we determine the constant needed to transform the KG equation into the GP equation. In Appendix B, we derive the hydrostatic equations describing spatially inhomogeneous relativistic SF/BEC clusters. In Appendix C, we recover from our formalism the nonrelativistic equations obtained in the framework of the GPP equations. In Appendix D we introduce the generalized KGP equations in which the gravitational potential is introduced by hand in the KG equation in a flat Friedmann-Lemaître-Robertson-Walker (FLRW) background space-time, and assumed to be given by a generalized Poisson equation where the source is the energy density $\epsilon$. We show that this model corresponds to the limit $\Phi / c^{2} \rightarrow+\infty$ of the KGE equations. In Appendices $\mathrm{E}$ G. we regroup all the elements necessary to make the numerical applications needed in the paper. A summary of our results can be found in the Proceedings of the X Mexican School on Gravitation and Mathematical Physics [112].

\section{THEORETICAL BACKGROUND}

\section{A. The Lagrangian of the scalar field}

We assume that DM can be described by a complex $\mathrm{SF}^{5}$ which is a continuous function of space and time

\footnotetext{
5 There are several reasons for considering a complex rather than a real SF [104]. Complex SFs have been invoked in many different sectors of elementary particle physics in relation to the
} 
defined at each point by $\varphi\left(x^{\mu}\right)=\varphi(x, y, z, t)$. The action of the relativistic $\mathrm{SF}$ is

$$
S_{\varphi}=\int d^{4} x \sqrt{-g} \mathcal{L}_{\varphi}
$$

where $\mathcal{L}_{\varphi}=\mathcal{L}_{\varphi}\left(\varphi, \varphi^{*}, \partial_{\mu} \varphi, \partial_{\mu} \varphi^{*}\right)$ is the Lagrangian density and $g=\operatorname{det}\left(g_{\mu \nu}\right)$ is the determinant of the metric tensor. We adopt the following generic Lagrangian density

$$
\mathcal{L}_{\varphi}=\frac{1}{2} g^{\mu \nu} \partial_{\mu} \varphi^{*} \partial_{\nu} \varphi-V\left(|\varphi|^{2}\right),
$$

which is written for a metric signature $(+,-,-,-)$. Specifically, we consider a SF potential of the form

$$
V\left(|\varphi|^{2}\right)=\frac{m^{2} c^{2}}{2 \hbar^{2}}|\varphi|^{2}+\frac{m^{2}}{2 \hbar^{4}} \lambda|\varphi|^{4},
$$

where the quadratic term is the rest-mass term and the quartic term is a self-interaction term. In view of future applications, we shall assume that the SF describes a BEC at $T=0$ in which all the particles are in the same ground state. In that case, the self-interacting constant $\lambda$ can be expressed in terms of the scattering length of the bosons $a_{s}$ and of their mass $m$ by $\lambda=4 \pi a_{s} \hbar^{2} / m$. The potential of the SF can be rewritten as

$$
V\left(|\varphi|^{2}\right)=\frac{m^{2} c^{2}}{2 \hbar^{2}}|\varphi|^{2}+\frac{2 \pi a_{s} m}{\hbar^{2}}|\varphi|^{4} .
$$

The self-interaction is repulsive when $a_{s}>0$ and attractive when $a_{s}<0$ [102]. The case of a general potential of the form $V\left(|\varphi|^{2}\right)$ is treated in 112 .

\section{B. The Klein-Gordon equation}

The equation of motion for the SF can be obtained from the principle of least action. Imposing $\delta S_{\varphi}=0$ for arbitrary variations $\delta \varphi$ and $\delta \varphi^{*}$, we obtain the EulerLagrange equation

$$
D_{\mu}\left[\frac{\partial \mathcal{L}_{\varphi}}{\partial\left(\partial_{\mu} \varphi\right)^{*}}\right]-\frac{\partial \mathcal{L}_{\varphi}}{\partial \varphi^{*}}=0
$$

where $D$ is the covariant derivative. For the generic Lagrangian (2), this leads to the KG equation

$$
\square \varphi+2 V\left(|\varphi|^{2}\right),_{\varphi^{*}}=0,
$$

where $\square$ is the d'Alembertian operator

$$
\square \equiv D_{\mu}\left(g^{\mu \nu} \partial_{\nu}\right)=\frac{1}{\sqrt{-g}} \partial_{\mu}\left(\sqrt{-g} g^{\mu \nu} \partial_{\nu}\right)
$$

Higgs mechanism responsible for mass generation. On the other hand, the $U(1)$ symmetry implies the dark matter particle number (charge) conservation [2]. Finally, complex scalar fields can form singular vortices leading to a rich dynamics of the halos when they rotate 72 . and

$$
V\left(|\varphi|^{2}\right),_{\varphi^{*}}=\frac{d V}{d|\varphi|^{2}} \varphi .
$$

For the specific SF potential (3), the KG equation takes the form

$$
\square \varphi+\frac{m^{2} c^{2}}{\hbar^{2}} \varphi+\frac{8 \pi a_{s} m}{\hbar^{2}}|\varphi|^{2} \varphi=0 .
$$

The d'Alembertian operator can be written in terms of the covariant derivative and of the Christoffel symbols as

$$
\begin{array}{r}
\square \varphi=D_{\mu}\left(g^{\mu \nu} \partial_{\nu} \varphi\right)=g^{\mu \nu} D_{\mu}\left(\partial_{\nu} \varphi\right) \\
=g^{\mu \nu} \partial_{\mu} \partial_{\nu} \varphi-g^{\mu \nu} \Gamma_{\mu \nu}^{\sigma} \partial_{\sigma} \varphi
\end{array}
$$

\section{The energy-momentum tensor}

Taking the variation of the SF action (1) with respect to $g^{\mu \nu}$, we get

$$
\delta S_{\varphi}=\frac{1}{2} \int d^{4} x \sqrt{-g} T_{\mu \nu} \delta g^{\mu \nu},
$$

where

$$
T_{\mu \nu}=2 \frac{\partial \mathcal{L}_{\varphi}}{\partial g^{\mu \nu}}-g_{\mu \nu} \mathcal{L}_{\varphi}
$$

is the energy-momentum tensor of the SF. For the generic Lagrangian (2), it takes the form

$$
\begin{array}{r}
T_{\mu \nu}=\frac{1}{2}\left(\partial_{\mu} \varphi^{*} \partial_{\nu} \varphi+\partial_{\nu} \varphi^{*} \partial_{\mu} \varphi\right) \\
-g_{\mu \nu}\left[\frac{1}{2} g^{\rho \sigma} \partial_{\rho} \varphi^{*} \partial_{\sigma} \varphi-V\left(|\varphi|^{2}\right)\right] .
\end{array}
$$

By analogy with the energy-momentum tensor of a perfect fluid, the energy density and the pressure tensor of the SF are defined by $\epsilon=T_{0}^{0}$ and $P_{i}^{j}=-T_{i}^{j}$. The conservation of the energy-momentum tensor, which results from the Noether theorem, writes $D_{\nu} T^{\mu \nu}=0$.

\section{The Einstein equations}

The Einstein-Hilbert action in general relativity is defined by

$$
S_{g}=\frac{c^{4}}{16 \pi G} \int d^{4} x \sqrt{-g} R,
$$

where $R$ is the Ricci scalar and $G$ is Newton's gravitational constant. Its variation with respect to $g^{\mu \nu}$ is given by [113]:

$$
\delta S_{g}=-\frac{c^{4}}{16 \pi G} \int d^{4} x \sqrt{-g}\left(R_{\mu \nu}-\frac{1}{2} g_{\mu \nu} R\right) \delta g^{\mu \nu},
$$


where $R_{\mu \nu}$ is the Ricci tensor. The total action (SF + gravity) is $S=S_{\varphi}+S_{g}$. The field equations can be obtained from the principle of least action. Imposing $\delta S=0$ for arbitrary variations in $g^{\mu \nu}$, and using Eqs. (11) and (15), we get the Einstein equations

$$
R_{\mu \nu}-\frac{1}{2} g_{\mu \nu} R=\frac{8 \pi G}{c^{4}} T_{\mu \nu}
$$

These are a set of 10 equations that describe the fundamental interaction between gravity and matter as a result of the curvature of space-time. The energy-momentum tensor $T^{\mu \nu}$ is the source of the gravitational field in the Einstein field equations of general relativity in the sense that it determines the metric $g^{\mu \nu}$. The conservation of the energy-momentum tensor is automatically included in the Einstein equations.

\section{THE KLEIN-GORDON-EINSTEIN EQUATIONS IN THE WEAK FIELD LIMIT}

In this paper, we study the KGE equations in the weak field limit $\Phi / c^{2} \ll 1$. The equations that we derive are valid at the order $O\left(\Phi / c^{2}\right)$. For $\Phi / c^{2} \rightarrow 0$, we obtain the generalized KGP equations (see Appendix D). Of course, the limit $\Phi / c^{2} \rightarrow 0$ is different from the nonrelativistic limit $c \rightarrow+\infty$ leading to the GPP equations (see Appendix C].

\section{A. The conformal Newtonian Gauge}

We work with the conformal Newtonian gauge [114. In general relativity, this gauge is a perturbed form of the FLRW line element. We assume that the Universe is flat in agreement with the observations of the cosmic microwave background (CMB). The general perturbed FLRW metric in the comoving frame has the form

$$
\begin{gathered}
d s^{2}=\left(1+2 \frac{\Psi}{c^{2}}\right) c^{2} d t^{2}-2 a(t) w_{i} c d t d x^{i} \\
-a(t)^{2}\left[\left(1-2 \frac{\Phi}{c^{2}}\right) \delta_{i j}+H_{i j}\right] d x^{i} d x^{j}
\end{gathered}
$$

where the perturbed quantities $\Psi / c^{2}, \Phi / c^{2}, w_{i}$, and $H_{i j}$ are all $\ll 1$. In this metric, $\Phi$ represents the gravitational potential of classical Newtonian gravity while $\Psi$ is the lapse function [114.

We consider the simplest form of the Newtonian gauge, only taking into account scalar perturbations which are the ones that contribute to the formation of structures in cosmology. Vector (which are supposed to be always small) vanish during cosmic inflation and tensor contributions (which account for gravitational waves) are neglected [115]. We also assume $\Psi=\Phi$, supposing a universe without anisotropic stress. Therefore, our line ele- ment is given by

$$
d s^{2}=c^{2}\left(1+2 \frac{\Phi}{c^{2}}\right) d t^{2}-a(t)^{2}\left(1-2 \frac{\Phi}{c^{2}}\right) \delta_{i j} d x^{i} d x^{j},
$$

where we recall that $\Phi / c^{2} \ll 1$. Working with this metric enables us to obtain exact equations at the order $O\left(\Phi / c^{2}\right)$ taking into account both relativistic and gravitational contributions inside an expanding Universe without having to introduce the gravitational potential $\Phi$ by hand.

\section{B. The Klein-Gordon equation}

Computing the d'Alembertian (7) with the Newtonian gauge (18), we obtain the $\mathrm{KG}$ equation

$$
\begin{aligned}
\frac{1}{c^{2}} \frac{\partial^{2} \varphi}{\partial t^{2}} & +\frac{3 H}{c^{2}} \frac{\partial \varphi}{\partial t}-\frac{1}{a^{2}}\left(1+\frac{4 \Phi}{c^{2}}\right) \Delta \varphi \\
& +2\left(1+2 \frac{\Phi}{c^{2}}\right) V_{\varphi_{\varphi^{*}}}-\frac{4}{c^{4}} \frac{\partial \Phi}{\partial t} \frac{\partial \varphi}{\partial t}=0
\end{aligned}
$$

where $H=\dot{a} / a$ is the Hubble constant and $\Delta=\vec{\nabla}^{2}$ is the usual Laplacian ( $\vec{\nabla}$ is the usual nabla operator). For the specific SF potential (4), the KG equation takes the form

$$
\begin{aligned}
& \frac{1}{c^{2}} \frac{\partial^{2} \varphi}{\partial t^{2}}+\frac{3 H}{c^{2}} \frac{\partial \varphi}{\partial t}-\frac{1}{a^{2}}\left(1+\frac{4 \Phi}{c^{2}}\right) \Delta \varphi \\
&- \frac{4}{c^{4}} \frac{\partial \Phi}{\partial t} \frac{\partial \varphi}{\partial t}+\left(1+\frac{2 \Phi}{c^{2}}\right) \frac{m^{2} c^{2}}{\hbar^{2}} \varphi \\
&+\left(1+\frac{2 \Phi}{c^{2}}\right) \frac{8 \pi a_{s} m}{\hbar^{2}}|\varphi|^{2} \varphi=0
\end{aligned}
$$

Using the expression 13 of the energy-momentum tensor, the energy density and the pressure are given by

$$
\begin{gathered}
\epsilon=T_{0}^{0}=\frac{1}{2 c^{2}}\left(1-\frac{2 \Phi}{c^{2}}\right)\left|\frac{\partial \varphi}{\partial t}\right|^{2} \\
+\frac{1}{2 a^{2}}\left(1+\frac{2 \Phi}{c^{2}}\right)|\vec{\nabla} \varphi|^{2}+V\left(|\varphi|^{2}\right) \\
P=-\frac{1}{3}\left(T_{1}^{1}+T_{2}^{2}+T_{3}^{3}\right)=\frac{1}{2 c^{2}}\left(1-\frac{2 \Phi}{c^{2}}\right)\left|\frac{\partial \varphi}{\partial t}\right|^{2} \\
-\frac{1}{6 a^{2}}\left(1+\frac{2 \Phi}{c^{2}}\right)|\vec{\nabla} \varphi|^{2}-V\left(|\varphi|^{2}\right) .
\end{gathered}
$$

\section{The Einstein equations}

The time-time component of the Einstein equations is

$$
R_{0}^{0}-\frac{1}{2} R=\frac{8 \pi G}{c^{4}} T_{0}^{0}
$$


With the Newtonian conformal gauge, the left hand side of Eq. (23) is given by

$$
R_{0}^{0}-\frac{1}{2} R=\frac{3 H^{2}}{c^{2}}+\frac{2}{a^{2} c^{2}} \Delta \Phi-\frac{6}{c^{4}} H\left(\frac{\partial \Phi}{\partial t}+H \Phi\right) .
$$

Therefore, the time-time component of the Einstein equations can be rewritten as

$$
\frac{\Delta \Phi}{4 \pi G a^{2}}=\frac{\epsilon}{c^{2}}-\frac{3 H^{2}}{8 \pi G}+\frac{3 H}{4 \pi G c^{2}}\left(\frac{\partial \Phi}{\partial t}+H \Phi\right) .
$$

Using the time-time component of the energy-momentum tensor, which represents the energy density $\epsilon$ given by Eq. (21), we get

$$
\begin{array}{r}
\frac{\Delta \Phi}{4 \pi G a^{2}}=\frac{1}{2 c^{4}}\left(1-\frac{2 \Phi}{c^{2}}\right)\left|\frac{\partial \varphi}{\partial t}\right|^{2} \\
+\frac{1}{2 a^{2} c^{2}}\left(1+\frac{2 \Phi}{c^{2}}\right)|\vec{\nabla} \varphi|^{2}+\frac{m^{2}}{2 \hbar^{2}}|\varphi|^{2} \\
+\frac{2 \pi a_{s} m}{\hbar^{2} c^{2}}|\varphi|^{4}-\frac{3 H^{2}}{8 \pi G}+\frac{3 H}{4 \pi G c^{2}}\left(\frac{\partial \Phi}{\partial t}+H \Phi\right) .
\end{array}
$$

Eqs. 20 and (26) form the KGE equations. This system of equations is closed because we consider a universe without anisotropic stress $(\Psi=\Phi)$. Otherwise, we need to write the other components of the Einstein equations.

\section{Spatially homogeneous scalar field}

For a spatially homogeneous SF with $\varphi_{b}(\vec{x}, t)=\varphi_{b}(t)$ and $\Phi_{b}(\vec{x}, t)=0$, the KG equation $(19)$ reduces to

$$
\frac{1}{c^{2}} \frac{d^{2} \varphi_{b}}{d t^{2}}+\frac{3 H}{c^{2}} \frac{d \varphi_{b}}{d t}+2 V\left(\left|\varphi_{b}\right|^{2}\right)_{\varphi_{b}^{*}}=0 .
$$

For the specific SF potential (4), we explicitly have

$$
\frac{1}{c^{2}} \frac{d^{2} \varphi_{b}}{d t^{2}}+\frac{3 H}{c^{2}} \frac{d \varphi_{b}}{d t}+\frac{m^{2} c^{2}}{\hbar^{2}} \varphi_{b}+\frac{8 \pi a_{s} m}{\hbar^{2}}\left|\varphi_{b}\right|^{2} \varphi_{b}=0 .
$$

For a homogeneous SF, the energy-momentum tensor is diagonal and isotropic, $T_{\nu}^{\mu}=\operatorname{diag}\left(\epsilon_{b},-P_{b},-P_{b},-P_{b}\right)$. The energy density $\epsilon_{b}(t)$ and the pressure $P_{b}(t)$ are given by

$$
\begin{aligned}
& \epsilon_{b}=\frac{1}{2 c^{2}}\left|\frac{d \varphi_{b}}{d t}\right|^{2}+V\left(\left|\varphi_{b}\right|^{2}\right), \\
& P_{b}=\frac{1}{2 c^{2}}\left|\frac{d \varphi_{b}}{d t}\right|^{2}-V\left(\left|\varphi_{b}\right|^{2}\right) .
\end{aligned}
$$

From these equations, we obtain the continuity equation

$$
\frac{d \epsilon_{b}}{d t}+3 H\left(\epsilon_{b}+P_{b}\right)=0
$$

which is one of the Friedmann equations [113. The other Friedmann equation is obtained from the Einstein equation (25) that reduces, when $\Phi_{b}=0$, to

$$
H^{2}=\frac{8 \pi G}{3 c^{2}} \epsilon_{b}
$$

This relation shows that the term $-3 H^{2} / 8 \pi G=-\epsilon_{b} / c^{2}$ in the Einstein equation (25) with $\Phi(\vec{x}, t) \neq 0$ plays the role of a neutralizing background. From Eqs. (31) and $(32)$, we easily obtain

$$
\frac{\ddot{a}}{a}=-\frac{4 \pi G}{3 c^{2}}\left(\epsilon_{b}+3 P_{b}\right)
$$

\section{E. The Gross-Pitaevskii-Einstein equations}

The KG equation without self-interaction can be viewed as a relativistic generalization of the Schrödinger equation. Similarly, the KG equation with a selfinteraction can be viewed as a relativistic generalization of the GP equation. In order to recover the Schrödinger and GP equations in the nonrelativistic limit $c \rightarrow+\infty$, we make the transformation [2]:

$$
\varphi(\vec{x}, t)=\frac{\hbar}{m} e^{-i m c^{2} t / \hbar} \psi(\vec{x}, t)
$$

The prefactor $\hbar / m$ is justified in Appendix A. Mathematically, we can always make this change of variables. However, we emphasize that it is only in the nonrelativistic limit $c \rightarrow+\infty$ that $\psi$ has the interpretation of a wave function, and that $|\psi|^{2}=\rho$ has the interpretation of a rest-mass density. In the relativistic regime, $\psi$ and $\rho=|\psi|^{2}$ do not have a clear physical interpretation. We will call them "pseudo wave function" and "pseudo restmass density". Nevertheless, it is perfectly legitimate to work with these variables and, as we shall see, the equations written in terms of these quantities take relatively simple forms that generalize naturally the nonrelativistic ones.

Substituting Eq. (34) in Eqs. 20) and 26), we obtain

$$
\begin{array}{r}
i \hbar \frac{\partial \psi}{\partial t}-\frac{\hbar^{2}}{2 m c^{2}} \frac{\partial^{2} \psi}{\partial t^{2}}-\frac{3}{2} H \frac{\hbar^{2}}{m c^{2}} \frac{\partial \psi}{\partial t} \\
+\frac{\hbar^{2}}{2 m a^{2}}\left(1+\frac{4 \Phi}{c^{2}}\right) \Delta \psi-m \Phi \psi \\
-\frac{4 \pi a_{s} \hbar^{2}}{m^{2}}\left(1+\frac{2 \Phi}{c^{2}}\right)|\psi|^{2} \psi+\frac{3}{2} i \hbar H \psi \\
+\frac{2 \hbar^{2}}{m c^{4}} \frac{\partial \Phi}{\partial t}\left(\frac{\partial \psi}{\partial t}-\frac{i m c^{2}}{\hbar} \psi\right)=0
\end{array}
$$




$$
\begin{array}{r}
\frac{\Delta \Phi}{4 \pi G a^{2}}=\left(1-\frac{\Phi}{c^{2}}\right)|\psi|^{2} \\
+\frac{\hbar^{2}}{2 m^{2} c^{4}}\left(1-\frac{2 \Phi}{c^{2}}\right)\left|\frac{\partial \psi}{\partial t}\right|^{2}+\frac{\hbar^{2}}{2 a^{2} m^{2} c^{2}}\left(1+\frac{2 \Phi}{c^{2}}\right)|\vec{\nabla} \psi|^{2} \\
+\frac{2 \pi a_{s} \hbar^{2}}{m^{3} c^{2}}|\psi|^{4}-\frac{\hbar}{m c^{2}}\left(1-\frac{2 \Phi}{c^{2}}\right) \operatorname{Im}\left(\frac{\partial \psi}{\partial t} \psi^{*}\right) \\
-\frac{3 H^{2}}{8 \pi G}+\frac{3 H}{4 \pi G c^{2}}\left(\frac{\partial \Phi}{\partial t}+H \Phi\right) .
\end{array}
$$

Eq. (35) can be interpreted as a generalized Schrödinger equation (in the absence of self-interaction $a_{s}=0$ ) or as a generalized GP equation (in the presence of selfinteraction $a_{s} \neq 0$ ). It is coupled to the Einstein equation (36).

The energy density and the pressure can be written as

$$
\begin{array}{r}
\epsilon=\frac{\hbar^{2}}{2 m^{2} c^{2}}\left(1-\frac{2 \Phi}{c^{2}}\right)\left|\frac{\partial \psi}{\partial t}\right|^{2} \\
-\frac{\hbar}{m}\left(1-\frac{2 \Phi}{c^{2}}\right) \operatorname{Im}\left(\frac{\partial \psi}{\partial t} \psi^{*}\right) \\
+\frac{\hbar^{2}}{2 a^{2} m^{2}}\left(1+\frac{2 \Phi}{c^{2}}\right)|\vec{\nabla} \psi|^{2} \\
+\frac{1}{2} c^{2}\left(1-\frac{2 \Phi}{c^{2}}\right)|\psi|^{2}+V\left(|\psi|^{2}\right) \\
P=\frac{\hbar^{2}}{2 m^{2} c^{2}}\left(1-\frac{2 \Phi}{c^{2}}\right)\left|\frac{\partial \psi}{\partial t}\right|^{2} \\
-\frac{\hbar}{m}\left(1-\frac{2 \Phi}{c^{2}}\right) \operatorname{Im}\left(\frac{\partial \psi}{\partial t} \psi^{*}\right) \\
-\frac{\hbar^{2}}{6 a^{2} m^{2}}\left(1+\frac{2 \Phi}{c^{2}}\right)|\vec{\nabla} \psi|^{2} \\
+\frac{1}{2} c^{2}\left(1-\frac{2 \Phi}{c^{2}}\right)|\psi|^{2}-V\left(|\psi|^{2}\right) .
\end{array}
$$

Eqs. (35) and (36) form the GPE equations. In the nonrelativistic limit $c \rightarrow+\infty$, we recover the GPP equations $\mathrm{C} 1 \mathrm{C} 2 \mathrm{C}$ of Appendix $\mathrm{C}$

\section{F. The hydrodynamic representation}

Important characteristics of the system are revealed by reformulating the KGE equations in the form of hydrodynamic equations. This can be done at the level of the GPE equations (35)-(36) via the Madelung transformation 105]. To that purpose, we write the pseudo wavefunction $\psi$ as

$$
\psi(\vec{x}, t)=\sqrt{\rho(\vec{x}, t)} e^{i S(\vec{x}, t) / \hbar},
$$

where $\rho=\psi \psi^{*}=|\psi|^{2}$ plays the role of a pseudo restmass density and $S=(1 / 2) i \hbar \ln \left(\psi^{*} / \psi\right)$ plays the role of a pseudo action. Following Madelung, we also define a pseudo velocity field as

$$
\vec{v}(\vec{x}, t)=\frac{\vec{\nabla} S}{m a},
$$

where the scale factor $a$ has been introduced in order to take into account the expansion of the Universe [97]. We note that this velocity field is irrotational.

Substituting Eqs. (39)- 40 in the GPE equations 35 (36), and separating real and imaginary parts, we obtain the system of hydrodynamic equations

$$
\begin{array}{r}
\frac{\partial \rho}{\partial t}+3 H \rho+\frac{1}{a} \vec{\nabla} \cdot(\rho \vec{v})=\frac{1}{m c^{2}} \frac{\partial}{\partial t}\left(\rho \frac{\partial S}{\partial t}\right) \\
+\frac{3 H \rho}{m c^{2}} \frac{\partial S}{\partial t}+\frac{4 \rho}{m c^{4}} \frac{\partial \Phi}{\partial t}\left(m c^{2}-\frac{\partial S}{\partial t}\right)-\frac{4 \Phi}{a c^{2}} \vec{\nabla} \cdot(\rho \vec{v}),
\end{array}
$$

$$
\begin{gathered}
\frac{\partial S}{\partial t}+\frac{(\vec{\nabla} S)^{2}}{2 m a^{2}}=-\frac{\hbar^{2}}{2 m c^{2}} \frac{\frac{\partial^{2} \sqrt{\rho}}{\partial t^{2}}}{\sqrt{\rho}} \\
+\left(1+\frac{4 \Phi}{c^{2}}\right) \frac{\hbar^{2}}{2 m a^{2}} \frac{\Delta \sqrt{\rho}}{\sqrt{\rho}}-\frac{2 \Phi}{m c^{2} a^{2}}(\vec{\nabla} S)^{2} \\
-m \Phi-\frac{4 \pi a_{s} \hbar^{2} \rho}{m^{2}}\left(1+\frac{2 \Phi}{c^{2}}\right) \\
+\frac{1}{2 m c^{2}}\left(\frac{\partial S}{\partial t}\right)^{2}-\left(3 H-\frac{4}{c^{2}} \frac{\partial \Phi}{\partial t}\right) \frac{\hbar^{2}}{4 m c^{2} \rho} \frac{\partial \rho}{\partial t}, \\
\frac{\partial \vec{v}}{\partial t}+H \vec{v}+\frac{1}{a}(\vec{v} \cdot \vec{\nabla}) \vec{v}=-\frac{\hbar^{2}}{2 a m^{2} c^{2}} \vec{\nabla}\left(\frac{\frac{\partial^{2}}{\partial t^{2}}}{\sqrt{\rho}}\right) \\
+\frac{\hbar^{2}}{2 m^{2} a^{3}}\left[\left(1+\frac{4 \Phi}{c^{2}}\right) \frac{\Delta \sqrt{\rho}}{\sqrt{\rho}}\right]-\frac{1}{a} \vec{\nabla} \Phi-\frac{1}{\rho a} \vec{\nabla} p \\
-\frac{8 \pi a_{s} \hbar^{2}}{a m^{3} c^{2}} \vec{\nabla}(\rho \Phi)-\frac{2}{a c^{2}} \vec{\nabla}\left(\Phi v^{2}\right)+\frac{1}{2 a m^{2} c^{2}} \vec{\nabla}\left[\left(\frac{\partial S}{\partial t}\right)^{2}\right] \\
-\frac{3 \hbar^{2}}{4 a m^{2} c^{2}} H \vec{\nabla}\left(\frac{1}{\rho} \frac{\partial \rho}{\partial t}\right)+\frac{\hbar^{2}}{a m^{2} c^{4}} \vec{\nabla}\left(\frac{\partial \Phi}{\partial t} \frac{\partial \rho}{\rho t}\right)
\end{gathered}
$$

$$
\begin{array}{r}
\frac{\Delta \Phi}{4 \pi G a^{2}}=\left(1-\frac{\Phi}{c^{2}}\right) \rho \\
+\frac{\hbar^{2}}{2 m^{2} c^{4}}\left(1-\frac{2 \Phi}{c^{2}}\right)\left[\frac{1}{4 \rho}\left(\frac{\partial \rho}{\partial t}\right)^{2}+\frac{\rho}{\hbar^{2}}\left(\frac{\partial S}{\partial t}\right)^{2}\right] \\
+\frac{\hbar^{2}}{2 a^{2} m^{2} c^{2}}\left(1+\frac{2 \Phi}{c^{2}}\right)\left[\frac{1}{4 \rho}(\vec{\nabla} \rho)^{2}+\frac{\rho}{\hbar^{2}}(\vec{\nabla} S)^{2}\right] \\
+\frac{2 \pi a_{s} \hbar^{2}}{m^{3} c^{2}}-\frac{1}{m c^{2}}\left(1-\frac{2 \Phi}{c^{2}}\right) \rho \frac{\partial S}{\partial t} \\
-\frac{3 H^{2}}{8 \pi G}+\frac{3 H}{4 \pi G c^{2}}\left(\frac{\partial \Phi}{\partial t}+H \Phi\right),
\end{array}
$$


where $p$ is a pseudo pressure given by the polytropic (quadratic) equation of state ${ }^{6}$

$$
p=\frac{2 \pi a_{s} \hbar^{2}}{m^{3}} \rho^{2} .
$$

We note that this equation of state coincides with the equation of state of a nonrelativistic BEC with a selfinteraction 102. This coincidence is not obvious because Eqs. 41-44 are valid in the relativistic regime. The interpretation of this equation of state is, however, not direct because $\rho$ and $p$ are a pseudo density and a pseudo pressure that coincide with the real density and the real pressure of a BEC only in the nonrelativistic limit $c \rightarrow$ $+\infty$.

The energy density and the pressure can be written in terms of hydrodynamic variables as

$$
\begin{aligned}
\epsilon= & \frac{\hbar^{2}}{2 m^{2} c^{2}}\left(1-\frac{2 \Phi}{c^{2}}\right)\left[\frac{1}{4 \rho}\left(\frac{\partial \rho}{\partial t}\right)^{2}+\frac{\rho}{\hbar^{2}}\left(\frac{\partial S}{\partial t}\right)^{2}\right] \\
& +\frac{\hbar^{2}}{2 a^{2} m^{2}}\left(1+\frac{2 \Phi}{c^{2}}\right)\left[\frac{1}{4 \rho}(\vec{\nabla} \rho)^{2}+\frac{\rho}{\hbar^{2}}(\vec{\nabla} S)^{2}\right] \\
- & \left(1-\frac{2 \Phi}{c^{2}}\right) \frac{\rho}{m} \frac{\partial S}{\partial t}+\frac{1}{2}\left(1-\frac{2 \Phi}{c^{2}}\right) \rho c^{2}+V(\rho), \\
P= & \frac{\hbar^{2}}{2 m^{2} c^{2}}\left(1-\frac{2 \Phi}{c^{2}}\right)\left[\frac{1}{4 \rho}\left(\frac{\partial \rho}{\partial t}\right)^{2}+\frac{\rho}{\hbar^{2}}\left(\frac{\partial S}{\partial t}\right)^{2}\right] \\
& -\frac{\hbar^{2}}{6 a^{2} m^{2}}\left(1+\frac{2 \Phi}{c^{2}}\right)\left[\frac{1}{4 \rho}(\vec{\nabla} \rho)^{2}+\frac{\rho}{\hbar^{2}}(\vec{\nabla} S)^{2}\right] \\
- & \left(1-\frac{2 \Phi}{c^{2}}\right) \frac{\rho}{m} \frac{\partial S}{\partial t}+\frac{1}{2}\left(1-\frac{2 \Phi}{c^{2}}\right) \rho c^{2}-V(\rho) .
\end{aligned}
$$

We note that, in general, the pressure $P$ defined by Eq. 477 is different from the pressure $p$ defined by Eq. 45. However, we shall find that they coincide for a spatially homogeneous SF.

The hydrodynamic equations (41)-44 have a clear physical interpretation. Eq. 41, corresponding to the imaginary part of the GPE equations, is the continuity equation. We note that $\int \rho d^{3} x$ is not conserved in the relativistic regime. However, we will see in Sec. V that Eq. (41) is consistent with the conservation of the charge of a spatially homogeneous SF. Eq. 42, corresponding to the real part of the GPE equations, is the Bernoulli or Hamilton-Jacobi equation. Eq. (43), obtained by taking the gradient of Eq. 42, is the momentum equation. Eq. (44) is the Einstein equation. We stress that the hydrodynamic equations (41)- 44 are equivalent to the GPE equations 35)-(36) which are themselves equivalent to the KGE equations 20 and 26. In the nonrelativistic limit $c \rightarrow+\infty$, we recover the quantum Euler-Poisson equations $\mathrm{C} 3 \mathrm{C}$ - 6 of Appendix C.

\footnotetext{
6 The equation of state $p(\rho)$ associated with a general potential of interaction of the form $V\left(|\varphi|^{2}\right)$ is given in [112].
}

\section{THE CASE OF A STATIC UNIVERSE: JEANS-TYPE INSTABILITY}

We first consider the case of a static Universe. This amounts to taking $H=0$ and $a=1$ in the previous equations.

\section{A. The fluid equations}

In a static Universe, the hydrodynamic equations (41)(44) reduce to

$$
\begin{aligned}
& \frac{\partial \rho}{\partial t}+\vec{\nabla} \cdot(\rho \vec{v})=\frac{1}{m c^{2}} \frac{\partial}{\partial t}\left(\rho \frac{\partial S}{\partial t}\right) \\
& +\frac{4 \rho}{m c^{4}} \frac{\partial \Phi}{\partial t}\left(m c^{2}-\frac{\partial S}{\partial t}\right)-\frac{4 \Phi}{c^{2}} \vec{\nabla} \cdot(\rho \vec{v}), \\
& \frac{\partial S}{\partial t}+\frac{(\vec{\nabla} S)^{2}}{2 m}=-\frac{\hbar^{2}}{2 m c^{2}} \frac{\frac{\partial^{2} \sqrt{\rho}}{\partial t^{2}}}{\sqrt{\rho}} \\
& +\left(1+\frac{4 \Phi}{c^{2}}\right) \frac{\hbar^{2}}{2 m} \frac{\Delta \sqrt{\rho}}{\sqrt{\rho}}-\frac{2 \Phi}{m c^{2}}(\vec{\nabla} S)^{2} \\
& -m \Phi-\frac{4 \pi a_{s} \hbar^{2} \rho}{m^{2}}\left(1+\frac{2 \Phi}{c^{2}}\right) \\
& +\frac{1}{2 m c^{2}}\left(\frac{\partial S}{\partial t}\right)^{2}+\frac{\hbar^{2}}{m c^{4} \rho} \frac{\partial \rho}{\partial t} \frac{\partial \Phi}{\partial t}, \\
& \frac{\partial \vec{v}}{\partial t}+(\vec{v} \cdot \vec{\nabla}) \vec{v}=-\frac{\hbar^{2}}{2 m^{2} c^{2}} \vec{\nabla}\left(\frac{\frac{\partial^{2} \sqrt{\rho}}{\partial t^{2}}}{\sqrt{\rho}}\right) \\
& +\frac{\hbar^{2}}{2 m^{2}} \vec{\nabla}\left[\left(1+\frac{4 \Phi}{c^{2}}\right) \frac{\Delta \sqrt{\rho}}{\sqrt{\rho}}\right]-\vec{\nabla} \Phi-\frac{1}{\rho} \vec{\nabla} p \\
& -\frac{8 \pi a_{s} \hbar^{2}}{m^{3} c^{2}} \vec{\nabla}(\rho \Phi)-\frac{2}{c^{2}} \vec{\nabla}\left(\Phi v^{2}\right)+\frac{1}{2 m^{2} c^{2}} \vec{\nabla}\left[\left(\frac{\partial S}{\partial t}\right)^{2}\right] \\
& +\frac{\hbar^{2}}{m^{2} c^{4}} \vec{\nabla}\left(\frac{\partial \Phi}{\partial t} \frac{1}{\rho} \frac{\partial \rho}{\partial t}\right) \\
& \frac{\Delta \Phi}{4 \pi G}=\left(1-\frac{\Phi}{c^{2}}\right) \rho \\
& +\frac{\hbar^{2}}{2 m^{2} c^{4}}\left(1-\frac{2 \Phi}{c^{2}}\right)\left[\frac{1}{4 \rho}\left(\frac{\partial \rho}{\partial t}\right)^{2}+\frac{\rho}{\hbar^{2}}\left(\frac{\partial S}{\partial t}\right)^{2}\right] \\
& +\frac{\hbar^{2}}{2 m^{2} c^{2}}\left(1+\frac{2 \Phi}{c^{2}}\right)\left[\frac{1}{4 \rho}(\vec{\nabla} \rho)^{2}+\frac{\rho}{\hbar^{2}}(\vec{\nabla} S)^{2}\right] \\
& +\frac{2 \pi a_{s} \hbar^{2}}{m^{3} c^{2}} \rho^{2}-\frac{1}{m c^{2}}\left(1-\frac{2 \Phi}{c^{2}}\right) \rho \frac{\partial S}{\partial t} .
\end{aligned}
$$




\section{B. Infinite homogeneous background}

We consider a homogeneous SF at rest extending in an infinite space. We have $\rho(\vec{x}, t)=\rho_{b}, \vec{v}_{b}(\vec{x}, t)=\overrightarrow{0}$, and $\Phi_{b}(\vec{x}, t)=0$. We must be careful, however, that the phase can depend on time: $S_{b}(\vec{x}, t)=S_{b}(t)$. Actually, the equation of continuity (48) implies that $d S_{b} / d t$ is a constant that we write $-E$ as it represents the energy of the homogeneous SF (with the opposite sign). Indeed, for a stationary state, the pseudo wavefunction of the SF writes $\psi_{b}(\vec{x}, t)=\sqrt{\rho_{b}} e^{-i E t / \hbar}$, so that $S_{b}(t)=-E t$. The energy of the $\mathrm{SF}$ is given by the Hamilton-Jacobi equation 49 which reduces to

$$
\frac{E^{2}}{2 m c^{2}}+E-\frac{4 \pi a_{s} \hbar^{2} \rho_{b}}{m^{2}}=0
$$

yielding

$$
E=m c^{2}\left[-1+\sqrt{1+\frac{8 \pi a_{s} \hbar^{2} \rho_{b}}{m^{3} c^{2}}}\right] .
$$

From Eqs. 46 and 47, the energy density and the pressure of the SF are

$$
\begin{gathered}
\epsilon_{b}=\rho_{b} c^{2}+\frac{\rho_{b}}{m}\left(\frac{E^{2}}{2 m c^{2}}+E\right)+\frac{2 \pi a_{s} \hbar^{2}}{m^{3}} \rho_{b}^{2}, \\
P_{b}=\frac{\rho_{b}}{m}\left(\frac{E^{2}}{2 m c^{2}}+E\right)-\frac{2 \pi a_{s} \hbar^{2}}{m^{3}} \rho_{b}^{2} .
\end{gathered}
$$

Combining Eq. 52 with Eqs. 54 and 55, we obtain

$$
\epsilon_{b}=\rho_{b} c^{2}+\frac{6 \pi a_{s} \hbar^{2}}{m^{3}} \rho_{b}^{2}, \quad P_{b}=\frac{2 \pi a_{s} \hbar^{2}}{m^{3}} \rho_{b}^{2} .
$$

We note that the pressure $P_{b}$ of a spatially homogeneous $\mathrm{SF}$ coincides with the pseudo pressure $p$ given by Eq. (45). We also note the identities

$$
\begin{aligned}
& \left(\frac{E}{m c^{2}}+1\right)^{2}=1+\frac{2 c_{s}^{2}}{c^{2}}, \\
& \left(\frac{E}{2 m c^{2}}+1\right) \frac{E}{m c^{2}}=\frac{c_{s}^{2}}{c^{2}},
\end{aligned}
$$

where

$$
c_{s}^{2}=P^{\prime}\left(\rho_{b}\right)=\frac{4 \pi a_{s} \hbar^{2} \rho_{b}}{m^{3}}
$$

is the square of the speed of sound in the homogeneous background. In terms of the speed of sound, the energy of the SF can be written as

$$
\frac{E}{m c^{2}}=-1+\sqrt{1+\frac{2 c_{s}^{2}}{c^{2}}} .
$$

Remark: The expression (53) of the energy $E$ of the SF can be directly obtained from the GP equation w5 with
$H=0, a=1$, and $\Phi_{b}=0$ by looking for a stationary solution of the form $\psi_{b}(\vec{x}, t)=\sqrt{\rho_{b}} e^{-i E t / \hbar}$. Then, using Eq. (34), implying $\varphi_{b}(\vec{x}, t)=\frac{\hbar}{m} \sqrt{\rho_{b}} e^{-i\left(m c^{2}+E\right) t / \hbar}$, we find that the total energy of the $\mathrm{SF}$, including its rest mass, is $E_{\text {tot }}=E+m c^{2}$. Using Eq. 53, we obtain

$$
E_{t o t}=m c^{2} \sqrt{1+\frac{8 \pi a_{s} \hbar^{2} \rho_{b}}{m^{3} c^{2}}} .
$$

\section{Linear wave equations}

We now slightly perturb the homogeneous SF and consider the evolution of the perturbations in the linear regime. This is the relativistic SF generalization of the classical Jeans problem [116]. As usual, we make the Jeans swindle [117]. We write $\rho(\vec{x}, t)=\rho_{b}+\delta \rho(\vec{x}, t)$ and $S(\vec{x}, t)=-E t+\delta S(\vec{x}, t)$ with $\delta \rho(\vec{x}, t) \ll 1$ and $\partial \delta S / \partial t(\vec{x}, t) \ll 1$. We also recall that $|\vec{v}(\vec{x}, t)| \ll 1$ and $\Phi(\vec{x}, t) \ll 1$. Introducing the notations

$$
\delta=\frac{\delta \rho}{\rho_{b}}, \quad \sigma=\frac{1}{m} \frac{\partial \delta S}{\partial t},
$$

we can write the linearized equations for the perturbations as

$$
\left(1+\frac{E}{m c^{2}}\right) \frac{\partial \delta}{\partial t}+\vec{\nabla} \cdot \vec{v}=\frac{1}{c^{2}} \frac{\partial \sigma}{\partial t}+\frac{4}{c^{2}} \frac{\partial \Phi}{\partial t}\left(1+\frac{E}{m c^{2}}\right)
$$

$$
\begin{array}{r}
\left(1+\frac{E}{m c^{2}}\right) \sigma=- \\
\frac{\hbar^{2}}{4 m^{2} c^{2}} \frac{\partial^{2} \delta}{\partial t^{2}}+\frac{\hbar^{2}}{4 m^{2}} \Delta \delta \\
-c_{s}^{2} \delta-\left(1+2 \frac{c_{s}^{2}}{c^{2}}\right) \Phi
\end{array}
$$

$$
\begin{gathered}
\left(1+\frac{E}{m c^{2}}\right) \frac{\partial \vec{v}}{\partial t}=-\frac{\hbar^{2}}{4 m^{2} c^{2}} \vec{\nabla}\left(\frac{\partial^{2} \delta}{\partial t^{2}}\right)+\frac{\hbar^{2}}{4 m^{2}} \vec{\nabla}(\Delta \delta) \\
-c_{s}^{2} \vec{\nabla} \delta-\left(1+2 \frac{c_{s}^{2}}{c^{2}}\right) \vec{\nabla} \Phi,
\end{gathered}
$$

$$
\begin{aligned}
& \frac{\Delta \Phi}{4 \pi G \rho_{b}}=\left(1+\frac{c_{s}^{2}}{c^{2}}\right) \delta-\frac{1}{c^{2}}\left(1+\frac{E}{m c^{2}}\right) \sigma-\frac{\Phi}{c^{2}} \\
& +\frac{E}{m c^{2}}\left(1+\frac{E}{2 m c^{2}}\right) \delta-\frac{2 E}{m c^{2}} \frac{\Phi}{c^{2}}\left(1+\frac{E}{2 m c^{2}}\right)
\end{aligned}
$$

Taking the time derivative of Eq. 63 and using $\frac{\partial}{\partial t} \nabla$. $\vec{v}=\Delta \sigma$, we get

$$
\frac{1}{c^{2}} \frac{\partial^{2} \sigma}{\partial t^{2}}-\Delta \sigma=\left(1+\frac{E}{m c^{2}}\right) \frac{\partial^{2} \delta}{\partial t^{2}}-\frac{4}{c^{2}} \frac{\partial^{2} \Phi}{\partial t^{2}}\left(1+\frac{E}{m c^{2}}\right) .
$$


Taking the Laplacian of Eq. (64), we obtain

$$
\begin{aligned}
\left(1+\frac{E}{m c^{2}}\right) \Delta \sigma & =\frac{\hbar^{2}}{4 m^{2}} \Delta\left(\Delta \delta-\frac{1}{c^{2}} \frac{\partial^{2} \delta}{\partial t^{2}}\right)-c_{s}^{2} \Delta \delta \\
& -\left(1+\frac{2 c_{s}^{2}}{c^{2}}\right) \Delta \Phi .
\end{aligned}
$$

Eqs. (67) and (68), together with Eq. (66), form a system of three coupled wave equations for the evolution of the perturbations $(\delta, \sigma, \Phi)$ in the linear regime.

\section{Dispersion relation and Jeans length}

Decomposing the perturbations in Fourier modes of the form $\delta(\vec{x}, t)=\delta_{k} \exp i(\vec{k} \cdot \vec{r}-\omega t), \sigma(\vec{x}, t)=$ $\sigma_{k} \exp i(\vec{k} \cdot \vec{r}-\omega t)$, and $\Phi(\vec{x}, t)=\Phi_{k} \exp i(\vec{k} \cdot \vec{r}-\omega t)$, we obtain after straightforward but lenghty calculations the dispersion relation

$$
\begin{array}{r}
\frac{\hbar^{2}}{4 m^{2} c^{4}} \omega^{4}-\left[\frac{1+\gamma}{3 \gamma+1} \frac{\hbar^{2} k^{2}}{2 m^{2} c^{2}}+1+\frac{3 c_{s}^{2}}{c^{2}}\right] \omega^{2} \\
+\frac{1}{1+3 \gamma}\left[(1-\gamma) \frac{\hbar^{2} k^{4}}{4 m^{2}}-\gamma k^{2} c^{2}+(1-3 \gamma) k^{2} c_{s}^{2}\right]=0
\end{array}
$$

where we have introduced the abbreviation

$$
\gamma=\frac{4 \pi G \rho_{b}}{k^{2} c^{2}}\left(1+\frac{2 c_{s}^{2}}{c^{2}}\right) .
$$

In the nonrelativistic limit $c \rightarrow+\infty$, the dispersion relation reduces to [57]:

$$
\omega^{2}=\frac{\hbar^{2} k^{4}}{4 m^{2}}+c_{s}^{2} k^{2}-4 \pi G \rho_{b} .
$$

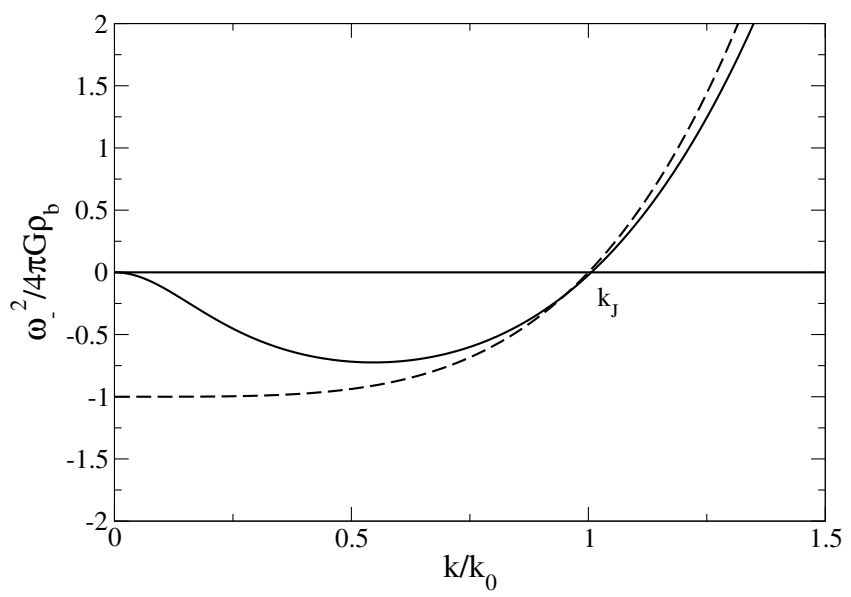

FIG. 1: Relativistic (solid line) and nonrelativistic (dashed line) dispersion relation. Here $k_{0}=\left(16 \pi G m^{2} \rho_{b} / \hbar^{2}\right)^{1 / 4}$. We have taken $a_{s}=0$ and $\chi=\left(4 \pi G \hbar^{2} \rho_{b} / m^{2} c^{4}\right)^{1 / 2}=0.05$.
Taking $\omega=0$ in Eq. 69, we find that the Jeans length $k_{J}$ is determined by the equation

$$
\begin{gathered}
\frac{\hbar^{2} k_{J}^{4}}{4 m^{2}}+\left[c_{s}^{2}-\frac{\pi \hbar^{2} G \rho_{b}}{m^{2} c^{2}}\left(1+\frac{2 c_{s}^{2}}{c^{2}}\right)\right] k_{J}^{2} \\
-4 \pi G \rho_{b}\left(1+\frac{2 c_{s}^{2}}{c^{2}}\right)\left(1+\frac{3 c_{s}^{2}}{c^{2}}\right)=0 .
\end{gathered}
$$

This is a second degree equation in $k_{J}^{2}$. Only the solution with the sign + has $k_{J}^{2}>0$. In the nonrelativistic limit $c \rightarrow+\infty$, Eq. (72) reduces to [57]:

$$
\frac{\hbar^{2} k_{J}^{4}}{4 m^{2}}+c_{s}^{2} k_{J}^{2}-4 \pi G \rho_{b}=0
$$

Eq. (69) is a second degree equation in $\omega^{2}$. We can show that the discriminant is positive so the relativistic dispersion relation has two branches $\omega_{ \pm}^{2}(k)$. For $k \rightarrow 0$,

$$
\begin{gathered}
\omega_{+}^{2}(k) \simeq \frac{4 m^{2} c^{4}}{\hbar^{2}}\left(1+\frac{3 c_{s}^{2}}{c^{2}}\right)+k^{2} c^{2}+\ldots, \\
\omega_{-}^{2}(k) \sim-\frac{1}{3} k^{2} c^{2} .
\end{gathered}
$$

For $k \rightarrow+\infty$,

$$
\omega_{ \pm}^{2}(k) \sim k^{2} c^{2}
$$

The branch $\omega_{+}^{2}(k)$ is always positive, corresponding to stable (oscillating) modes. The branch $\omega_{-}^{2}(k)$ starts from 0 , decreases, reaches a minimum, increases, vanishes at $k=k_{J}$, and finally tends to $+\infty$ (see Fig. 11). The modes are stable (oscillating) for $k>k_{J}$ and unstable (growing) for $k<k_{J}$. However, in the relativistic case, the system is stabilized at very large scales since $\omega_{-}^{2}(k) \rightarrow$ 0 for $k \rightarrow 0$ while in the nonrelativistic case $\omega^{2}(0)=$ $-4 \pi G \rho_{b}<0$. This shows that the limits $c \rightarrow+\infty$ and $k \rightarrow 0$ do not commute. We shall encounter this largescale stabilization again in Secs. VIB and VII G.

\section{COSMOLOGICAL EVOLUTION OF A SPATIALLY HOMOGENEOUS SCALAR FIELD}

We consider the evolution of a universe induced solely by a spatially homogeneous SF. In the comoving frame, we have $\rho(\vec{x}, t)=\rho_{b}(t), \vec{v}_{b}(\vec{x}, t)=\overrightarrow{0}, \Phi_{b}(\vec{x}, t)=0$, and $S_{b}(\vec{x}, t)=S_{b}(t)$. We introduce the notation $E(t)=$ $-d S_{b} / d t$ which can be considered as the time-dependent energy of the spatially homogeneous SF in the comoving frame. The wavefunction of the $\mathrm{SF}$ is $\psi_{b}(\vec{x}, t)=$ $\sqrt{\rho_{b}(t)} e^{-(i / \hbar) \int E(t) d t}$. Using Eq. (34), we have $\varphi_{b}(\vec{x}, t)=$ $\frac{\hbar}{m} \sqrt{\rho_{b}(t)} e^{-(i / \hbar)\left(m c^{2} t+\int E(t) d t\right)}$ so the total energy of the $\mathrm{SF}$, including its rest mass, is $E_{\text {tot }}(t)=E(t)+m c^{2}$. 


\section{A. Hydrodynamic equations for a homogeneous scalar field}

For a spatially homogeneous SF, the hydrodynamic equations (41)-44 reduce to

$$
\begin{gathered}
\frac{d \rho_{b}}{d t}+3 H \rho_{b}=-\frac{1}{m c^{2}} \frac{d}{d t}\left(\rho_{b} E\right)-\frac{3 H \rho_{b}}{m c^{2}} E \\
\left(\frac{E}{2 m c^{2}}+1\right) E=\frac{\hbar^{2}}{2 m c^{2}} \frac{\frac{d^{2} \sqrt{\rho_{b}}}{d t^{2}}}{\sqrt{\rho_{b}}}+\frac{4 \pi a_{s} \hbar^{2} \rho_{b}}{m^{2}} \\
+\frac{3 H \hbar^{2}}{4 m c^{2} \rho_{b}} \frac{d \rho_{b}}{d t} \\
\begin{array}{c}
\frac{3 H^{2}}{8 \pi G}=\rho_{b}+\frac{\hbar^{2}}{2 m^{2} c^{4}}\left[\frac{1}{4 \rho_{b}}\left(\frac{d \rho_{b}}{d t}\right)^{2}+\frac{\rho_{b}}{\hbar^{2}} E^{2}\right] \\
+\frac{2 \pi a_{s} \hbar^{2}}{m^{3} c^{2}} \rho_{b}^{2}+\frac{E}{m c^{2}} \rho_{b} .
\end{array}
\end{gathered}
$$

In terms of the total energy $E_{\text {tot }}(t)=E(t)+m c^{2}$, the equation of continuity (77) becomes

$$
\frac{1}{\rho_{b}} \frac{d \rho_{b}}{d t}+\frac{3}{a} \frac{d a}{d t}+\frac{1}{E_{\mathrm{tot}}} \frac{d E_{\mathrm{tot}}}{d t}=0 .
$$

It can be rewritten as a conservation law:

$$
\frac{d}{d t}\left(E_{\text {tot }} \rho_{b} a^{3}\right)=0
$$

Therefore, the total energy is exactly given by

$$
\frac{E_{\mathrm{tot}}}{m c^{2}}=\frac{Q m}{\rho_{b} a^{3}},
$$

where $Q$ is a constant. This conservation law was found by $\mathrm{Gu}$ and Hwang 118 directly from the KG equation (see also Appendix B of 104). It can be shown that $Q=\int J^{0} d^{3} x$ represents the conserved charge density of the complex SF.

The energy density and the pressure of a homogeneous SF are

$$
\begin{array}{r}
\epsilon_{b}=\frac{\hbar^{2}}{8 m^{2} c^{2}} \frac{1}{\rho_{b}}\left(\frac{d \rho_{b}}{d t}\right)^{2}+ \\
+\frac{\rho_{b}}{m} E\left(1+\frac{E}{2 m c^{2}}\right) \\
+\rho_{b} c^{2}+\frac{2 \pi a_{s} \hbar^{2}}{m^{3}} \rho_{b}^{2}, \\
P=\frac{\hbar^{2}}{8 m^{2} c^{2}} \frac{1}{\rho_{b}}\left(\frac{d \rho_{b}}{d t}\right)^{2}+\frac{\rho_{b}}{m} E\left(1+\frac{E}{2 m c^{2}}\right) \\
-\frac{2 \pi a_{s} \hbar^{2}}{m^{3}} \rho_{b}^{2} .
\end{array}
$$

Equations (78), (79) and 82 determine the complete evolution of a universe induced by a spatially homoge- neous SF. ${ }^{7}$ Working directly on the homogeneous $\mathrm{KG}$ equation, Li et al. 104 have shown that a universe filled with a relativistic complex SF first undergoes an intrinsic stiff matter era, followed by a radiation era due to its selfinteraction, before finally entering in the matter era. The stiff matter era occurs when the SF oscillations are slower than the Hubble expansion while the radiation and matter eras occur when the SF oscillations are faster than the Hubble expansion. Since we propose in this paper to use hydrodynamic equations instead of the KG equation, it is important to show, for self-consistency, that these different regimes can be directly obtained from the hydrodynamic equations (78, 79 and 82 . One advantage of the hydrodynamic representation is that we do not have to make averagings over the oscillations of the SF as in [104.

\section{B. Stiff matter era}

At early times $(a \rightarrow 0, t \rightarrow 0)$, we can approximate Eqs. (78) and (79) by $^{8}$

$$
\begin{gathered}
\frac{\hbar^{2}}{m^{2} c^{4}} \frac{\frac{d^{2} \sqrt{\rho_{b}}}{d t^{2}}}{\sqrt{\rho_{b}}}+\frac{3 H \hbar^{2}}{2 m^{2} c^{4} \rho_{b}} \frac{d \rho_{b}}{d t}=0, \\
\frac{3 H^{2}}{8 \pi G}=\frac{\hbar^{2}}{8 m^{2} c^{4} \rho_{b}}\left(\frac{d \rho_{b}}{d t}\right)^{2} .
\end{gathered}
$$

Recalling that $H=\dot{a} / a$, Eq. 86 can be integrated into

$$
\rho_{b} \sim \frac{3 m^{2} c^{4}}{4 \pi G \hbar^{2}}(-\ln a)^{2} .
$$

Substituting Eq. 87) in Eq. 85, we obtain

$$
\frac{d H}{d t}+3 H^{2}=0
$$

which yields

$$
H=\frac{1}{3 t}, \quad a \propto t^{1 / 3}
$$

\footnotetext{
7 A remark may be in order. A SF $\varphi_{b}(t)$ oscillates in time because it has a phase $S_{b}(t)$. The kinetic and potential energies $\dot{\varphi}_{b}^{2}$ and $V\left(\varphi_{b}^{2}\right)$ of a real SF also present oscillations because they keep track of the phase $S_{b}(t)$ of the SF $53,97,98$. By contrast, the kinetic and potential energies $\left|\dot{\varphi}_{b}\right|^{2}$ and $V\left(\left|\varphi_{b}\right|^{2}\right)$ of a complex $\mathrm{SF}$ do not oscillate in time. It is not clear how one can measure the oscillations of a complex SF because we do not have directly access to field variables such as $\varphi_{b}(t)$ but rather to hydrodynamic variables such as $\epsilon_{b}(t), \rho_{b}(t)$, and $P_{b}(t)$. In this sense, the hydrodynamic representation of the SF may be more physical than the KG equation itself.

8 We can check a posteriori that the terms that have been neglected are indeed negligible when $a \rightarrow 0$ and $t \rightarrow 0$.
} 
Substituting Eq. 89 in Eq. 87 we find that the pseudo rest-mass density behaves as

$$
\rho_{b} \sim \frac{m^{2} c^{4}}{12 \pi G \hbar^{2}}(-\ln t)^{2} .
$$

According to Eq. 82 the energy of the homogeneous SF behaves as

$$
\frac{E}{m c^{2}} \sim \frac{4 \pi Q G \hbar^{2}}{3 m c^{4}} \frac{1}{a^{3}(-\ln a)^{2}} \propto \frac{1}{(-\ln t)^{2} t} .
$$

Finally, the energy density and the pressure of the homogeneous SF behave as

$$
P_{b}=\epsilon_{b}=\frac{3 H^{2} c^{2}}{8 \pi G}=\frac{c^{2}}{24 \pi G t^{2}} \propto \frac{1}{a^{6}} .
$$

These results can be straightforwardly obtained from the $\mathrm{KG}$ equation in the regime where the SF oscillations are slower than the Hubble expansion 104. Indeed, neglecting the potential energy in Eqs. 29 and 30 , we get $P=\epsilon$ which is the equation of state of a stiff fluid for which the speed of sound $c_{s}=\sqrt{P^{\prime}(\epsilon)} c$ is equal to the speed of light $\left(c_{s}=c\right)$. Substituting this equation of state in the Friedmann equations (31) and (32), we directly obtain Eq. (92). Therefore, when $t \rightarrow 0$, a relativistic SF behaves as a stiff fluid 104. This stiff matter era is independent on the scattering length $a_{s}$ of the bosons. Therefore, it exists for both self-interacting and non-interacting SFs.

\section{Radiation and matter eras}

At later times, following the stiff matter era, we can approximate Eqs. (78) and (79) by ${ }^{9}$

$$
\begin{gathered}
\left(\frac{E}{2 m c^{2}}+1\right) E=\frac{4 \pi a_{s} \hbar^{2} \rho_{b}}{m^{2}} \\
\frac{3 H^{2}}{8 \pi G}=\rho_{b}+\frac{2 \pi a_{s} \hbar^{2}}{m^{3} c^{2}} \rho_{b}^{2}+\frac{E}{m c^{2}}\left(\frac{E}{2 m c^{2}}+1\right) \rho_{b} .
\end{gathered}
$$

Substituting Eq. (93) in Eq. (94), we obtain

$$
\frac{3 H^{2}}{8 \pi G}=\rho_{b}+\frac{6 \pi a_{s} \hbar^{2}}{m^{3} c^{2}} \rho_{b}^{2} .
$$

Eq. (93) is the same as Eq. (52) in the static case, so we still have the identity (53), where now $E$ and $\rho_{b}$ depend on time. Combining Eqs. (53) and 82 we get

$$
\rho_{b}\left(1+\frac{8 \pi a_{s} \hbar^{2} \rho_{b}}{m^{3} c^{2}}\right)^{1 / 2}=\frac{Q m}{a^{3}} .
$$

\footnotetext{
9 These equations correspond to the terms that have been neglected in Sec. $\mathrm{VB}$ and that now become important.
}

Equations (95) and 96 determine the evolution of the Universe induced by the homogeneous SF in the regime where its oscillations are faster than the Hubble expansion.

The energy density and the pressure are given by

$$
\epsilon_{b}=\rho_{b} c^{2}+\frac{6 \pi a_{s} \hbar^{2}}{m^{3}} \rho_{b}^{2}, \quad P_{b}=\frac{2 \pi a_{s} \hbar^{2}}{m^{3}} \rho_{b}^{2} .
$$

We note that the pressure $P_{b}(t)$ of a spatially homogeneous scalar field coincides with the pseudo pressure $p(t)$ given by Eq. 45. From Eq. (97) we get

$$
P_{b}=\frac{m^{3} c^{4}}{72 \pi a_{s} \hbar^{2}}\left(\sqrt{1+\frac{24 \pi a_{s} \hbar^{2}}{m^{3} c^{4}}} \epsilon_{b}-1\right)^{2} .
$$

This is the equation of state obtained by Colpi et al. [14] in the context of boson stars (see also [32, 46, 104]).

We can now determine the asymptotic behavior of the homogeneous SF in the regime where its oscillations are faster than the Hubble expansion, i.e. in the regime that follows the stiff matter era.

(i) At late times $t \rightarrow+\infty\left(\rho_{b} \rightarrow 0, a \rightarrow+\infty\right)$, we get

$$
\begin{gathered}
\rho_{b} \sim \frac{Q m}{a^{3}}, \quad \frac{E}{m c^{2}} \sim \frac{4 \pi a_{s} \hbar^{2} Q}{m^{2} c^{2} a^{3}} . \\
\frac{3 H^{2}}{8 \pi G} \sim \rho_{b} \sim \frac{Q m}{a^{3}}, \quad a \sim\left(6 \pi G Q m t^{2}\right)^{1 / 3}, \\
\epsilon_{b} \sim \rho_{b} c^{2}, \quad P_{b} \sim \frac{2 \pi a_{s} \hbar^{2}}{m^{3} c^{4}} \epsilon_{b}^{2} \simeq 0 .
\end{gathered}
$$

The SF/BEC universe behaves as a pressureless matter fluid.

(ii) At early times $t \rightarrow 0\left(\rho_{b} \rightarrow+\infty, a \rightarrow 0\right)$, we get

$$
\rho_{b} \sim\left(\frac{Q^{2} m^{5} c^{2}}{8 \pi a_{s} \hbar^{2}}\right)^{1 / 3} \frac{1}{a^{2}}, \quad \frac{E}{m c^{2}} \sim\left(\frac{8 \pi a_{s} \hbar^{2} Q}{m^{2} c^{2}}\right)^{1 / 3} \frac{1}{a},
$$

$$
\frac{3 H^{2}}{8 \pi G} \sim \frac{6 \pi a_{s} \hbar^{2}}{m^{3} c^{2}} \rho_{b}^{2} \sim \frac{3}{2}\left(\frac{Q^{4} \pi m a_{s} \hbar^{2}}{c^{2}}\right)^{1 / 3} \frac{1}{a^{4}}
$$

$$
\begin{gathered}
a \sim 2\left(\frac{\pi^{4} G^{3} Q^{4} m a_{s} \hbar^{2}}{c^{2}}\right)^{1 / 12} t^{1 / 2}, \\
\epsilon_{b} \sim \frac{6 \pi a_{s} \hbar^{2}}{m^{3}} \rho_{b}^{2}, \quad P_{b} \sim \frac{1}{3} \epsilon_{b} .
\end{gathered}
$$

The SF/BEC universe behaves like radiation. As emphasized by Li et al. 104, the radiation era is due to the self-interaction of the SF $\left(a_{s} \neq 0\right)$. There is no such phase for a non-interacting $\operatorname{SF}\left(a_{s}=0\right)$. 
Remark: the case of a SF with an attractive selfinteraction $\left(a_{s}<0\right)$ is theoretically interesting because it leads to a model of universe without initial Big Bang singularity. At $t=0$, the universe has a finite scale factor $a_{*}=\left(12 \sqrt{3} \pi Q\left|a_{s}\right| \hbar^{2} / m^{2} c^{2}\right)^{1 / 3}$ and a finite density $\rho_{*}=m^{3} c^{2} / 12 \pi\left|a_{s}\right| \hbar^{2}$. Two evolutions are possible: (i) a normal evolution in which the density decreases as the scale factor increases until the system enters in the matter era, and (ii) a phantom-like evolution in which the density increases as the scale factor increases until the universe enters in a de Sitter era with a constant density $\rho_{\max }=m^{3} c^{2} / 8 \pi\left|a_{s}\right| \hbar^{2}$. The choice of the evolution between these two behaviors cannot be decided a priori. This interesting dynamical system will be studied in a specific paper (in preparation).

\section{EVOLUTION OF THE PERTURBATIONS IN THE EXPANDING UNIVERSE}

We now slightly perturb the homogeneous SF and consider the evolution of the perturbations in the linear regime.

\section{A. Linearized equations}

The linearized hydrodynamic equations obtained from Eqs. 41)-(44) describing the evolution of small inhomogeneities in the expanding Universe are

$$
\begin{aligned}
& \left(1+\frac{E}{m c^{2}}\right) \frac{\partial \delta}{\partial t}+\frac{1}{a} \vec{\nabla} \cdot \vec{v}=\frac{1}{c^{2}} \frac{\partial \sigma}{\partial t} \\
& +\frac{1}{c^{2}}\left(\frac{1}{\rho_{b}} \frac{d \rho_{b}}{d t}+3 H\right) \sigma+\frac{4}{c^{2}} \frac{\partial \Phi}{\partial t}\left(1+\frac{E}{m c^{2}}\right), \\
& \left(1+\frac{E}{m c^{2}}\right) \sigma=\frac{\hbar^{2}}{4 m^{2} a^{2}} \Delta \delta-c_{s}^{2} \delta \\
& -\frac{\hbar^{2}}{4 m^{2} c^{2}} \frac{1}{\sqrt{\rho_{b}}}\left[\frac{\partial^{2}}{\partial t^{2}}\left(\sqrt{\rho_{b}} \delta\right)-\delta \frac{d^{2}}{d t^{2}}\left(\sqrt{\rho_{b}}\right)\right] \\
& -\frac{3 H \hbar^{2}}{4 m^{2} c^{2}} \frac{\partial \delta}{\partial t}-\left(1+\frac{2 c_{s}^{2}}{c^{2}}\right) \Phi+\frac{\hbar^{2}}{m^{2} c^{4}} \frac{1}{\rho_{b}} \frac{d \rho_{b}}{d t} \frac{\partial \Phi}{\partial t}, \\
& \frac{\partial \vec{v}}{\partial t}+H \vec{v}=\frac{\hbar^{2}}{4 m^{2} a^{3}} \vec{\nabla}(\Delta \delta)-\frac{1}{a} c_{s}^{2} \vec{\nabla} \delta \\
& -\frac{\hbar^{2}}{4 a m^{2} c^{2}} \frac{1}{\sqrt{\rho_{b}}} \nabla \cdot\left[\frac{\partial^{2}}{\partial t^{2}}\left(\sqrt{\rho_{b}} \delta\right)-\delta \frac{d^{2}}{d t^{2}}\left(\sqrt{\rho_{b}}\right)\right] \\
& -\frac{E}{a m c^{2}} \nabla \sigma-\frac{3 \hbar^{2} H}{4 a m^{2} c^{2}} \nabla\left(\frac{\partial \delta}{\partial t}\right) \\
& -\frac{1}{a}\left(1+\frac{2 c_{s}^{2}}{c^{2}}\right) \vec{\nabla} \Phi+\frac{\hbar^{2}}{a m^{2} c^{4}} \frac{1}{\rho_{b}} \frac{d \rho_{b}}{d t} \nabla \frac{\partial \Phi}{\partial t},
\end{aligned}
$$

$$
\begin{array}{r}
\frac{\Delta \Phi}{4 \pi G \rho_{b} a^{2}}=\left(1+\frac{c_{s}^{2}}{c^{2}}\right) \delta-\frac{1}{c^{2}}\left(\frac{E}{m c^{2}}+1\right) \sigma \\
+\frac{E}{m c^{2}}\left(\frac{E}{2 m c^{2}}+1\right) \delta \\
+\frac{\hbar^{2}}{8 m^{2} c^{4} \rho_{b}^{2}}\left[2 \rho_{b} \frac{\partial \delta}{\partial t}+\delta \frac{d \rho_{b}}{d t}\right] \frac{d \rho_{b}}{d t} \\
-\frac{\Phi}{c^{2}}-\frac{\hbar^{2}}{4 m^{2} c^{4}} \frac{\Phi}{c^{2}} \frac{1}{\rho_{b}^{2}}\left(\frac{d \rho_{b}}{d t}\right)^{2} \\
-\frac{E}{m c^{2}}\left(\frac{E}{2 m c^{2}}+1\right) \frac{2 \Phi}{c^{2}}+\frac{\partial \Phi}{4 \pi G \rho_{b} c^{2}}\left(\frac{\partial \Phi}{\partial t}+H \Phi\right),
\end{array}
$$

where $\delta, \sigma$ and $c_{s}^{2}$ are defined as in Eqs. 59 and 62 where $\rho_{b}(t)$ is now time-dependent.

\section{B. Approximate equation for the density contrast}

A detailed study of the exact linearized equations 106-109 derived previously will be the subject of a future work. Here, we derive an approximate equation for the density contrast $\delta(\vec{x}, t)$ by neglecting the relativistic terms that involve a time derivative, i.e. terms like $\frac{1}{c^{\alpha}} \frac{\partial^{\beta}}{\partial t^{\beta}}$. In this way, we simplify the equation for $\delta(\vec{x}, t)$ without altering the expression of the relativistic Jeans length.

With this approximation, the linearized equations (106)-109 become

$$
\begin{gathered}
\frac{\partial \delta}{\partial t}+\frac{1}{a} \vec{\nabla} \cdot \vec{v}=0 \\
\frac{1}{c^{2}}\left(1+\frac{E}{m c^{2}}\right) \sigma=\frac{\hbar^{2}}{4 m^{2} c^{2} a^{2}} \Delta \delta-\left(1+\frac{2 c_{s}^{2}}{c^{2}}\right) \frac{\Phi}{c^{2}}-\frac{c_{s}^{2}}{c^{2}} \delta \\
\frac{\partial \vec{v}}{\partial t}+H \vec{v}=\frac{\hbar^{2}}{4 m^{2} a^{3}} \vec{\nabla}(\Delta \delta)-\frac{1}{a}\left(1+\frac{2 c_{s}^{2}}{c^{2}}\right) \vec{\nabla} \Phi-\frac{1}{a} c_{s}^{2} \vec{\nabla} \delta \\
\frac{\Delta \Phi}{4 \pi G \rho_{b} a^{2}}=\left(1+\frac{c_{s}^{2}}{c^{2}}\right) \delta-\frac{1}{c^{2}}\left(\frac{E}{m c^{2}}+1\right) \sigma \\
+\frac{E}{m c^{2}}\left(\frac{E}{2 m c^{2}}+1\right) \delta-\frac{E}{m c^{2}}\left(1+\frac{E}{2 m c^{2}}\right) \frac{2 \Phi}{c^{2}} \\
-\frac{\Phi}{c^{2}}+\frac{3 H^{2}}{4 \pi G \rho_{b} c^{2}} \Phi .
\end{gathered}
$$

Taking the time derivative of Eq. 110 and the divergence of Eq. 112, we obtain

$$
\begin{aligned}
\frac{\partial^{2} \delta}{\partial t^{2}}+2 H \frac{\partial \delta}{\partial t}= & \frac{c_{s}^{2}}{a^{2}} \Delta \delta-\frac{\hbar^{2}}{4 m^{2} a^{4}} \Delta^{2} \delta \\
& +\frac{1}{a^{2}}\left(1+\frac{2 c_{s}^{2}}{c^{2}}\right) \Delta \Phi .
\end{aligned}
$$


Substituting Eq. 111 in Eq. 113, we get

$$
\begin{array}{r}
\frac{\Delta \Phi}{4 \pi G \rho_{b} a^{2}}=\left(1+2 \frac{c_{s}^{2}}{c^{2}}\right) \delta-\frac{\hbar^{2}}{4 m^{2} c^{2} a^{2}} \Delta \delta \\
+\left(1+\frac{2 c_{s}^{2}}{c^{2}}\right) \frac{\Phi}{c^{2}}+\frac{E}{m c^{2}}\left(\frac{E}{2 m c^{2}}+1\right) \delta \\
-\frac{E}{m c^{2}}\left(\frac{E}{2 m c^{2}}+1\right) \frac{2 \Phi}{c^{2}}-\frac{\Phi}{c^{2}}+\frac{3 H^{2}}{4 \pi G \rho_{b} c^{2}} \Phi .
\end{array}
$$

Decomposing the perturbations in (spatial) Fourier modes, and using the identities (57) and (58) that remain valid in the present context where now $E(t)$ and $c_{s}^{2}(t)$ are time-dependent, we obtain a closed equation for $\delta_{k}(t)$ :

$$
\begin{array}{r}
\frac{d^{2} \delta_{k}}{d t^{2}}+2 H \frac{d \delta_{k}}{d t}+\left[\frac{\hbar^{2} k^{4}}{4 m^{2} a^{4}}+\frac{c_{s}^{2}}{a^{2}} k^{2}\right. \\
\left.-\frac{4 \pi G \rho_{b}}{1+\frac{3 H^{2} a^{2}}{k^{2} c^{2}}}\left(1+\frac{2 c_{s}^{2}}{c^{2}}\right)\left(1+\frac{3 c_{s}^{2}}{c^{2}}+\frac{\hbar^{2} k^{2}}{4 m^{2} c^{2} a^{2}}\right)\right] \delta_{k}=0
\end{array}
$$

In a static universe $(a=1, H=0)$, taking $\ddot{\delta}_{k}=0$ (i.e. $\omega=0)$, we can check that Eq. (116) reduces to Eq. 72 that determines the relativistic Jeans length. In the nonrelativistic limit $c \rightarrow+\infty$, the equation for the density contrast reduces to [97, 100]:

$$
\frac{d^{2} \delta_{k}}{d t^{2}}+2 H \frac{d \delta_{k}}{d t}+\left(\frac{\hbar^{2} k^{4}}{4 m^{2} a^{4}}+\frac{c_{s}^{2}}{a^{2}} k^{2}-4 \pi G \rho_{b}\right) \delta_{k}=0
$$

Because of the term appearing in the denominator of Eq. (116), the limits $k \rightarrow 0$ and $c \rightarrow+\infty$ do not commute. If we take the limit $c \rightarrow+\infty$ before the limit $k \rightarrow 0$, Eq. 116 reduces to

$$
\frac{d^{2} \delta}{d t^{2}}+2 H \frac{d \delta}{d t}-4 \pi G \rho_{b} \delta=0
$$

which is equivalent to the CDM model where the evolution of the perturbations is due only to the gravitational force. By contrast, if we take the limit $k \rightarrow 0$ before the limit $c \rightarrow+\infty$, Eq. 116 reduces to

$$
\frac{d^{2} \delta}{d t^{2}}+2 H \frac{d \delta}{d t}=0
$$

in which the gravitational force has "disappeared". This shows that the term appearing in the denominator of Eq. 116, which is purely relativistic, acts as an attenuation factor. Actually, this term can be written as $1+\left(\lambda / \lambda_{H}\right)^{2}$ where $\lambda=2 \pi / k$ is the wavelength of the perturbation and

$$
\lambda_{H}=2 \pi \frac{c}{\sqrt{3} H a}
$$

is the cosmological horizon, or Hubble length. ${ }^{10}$ This is the distance traveled by a photon with the velocity $c$ during the Hubble time $H^{-1}$. The cosmological horizon sets the size of the observable universe. When $\lambda \ll \lambda_{H}$, which is the most relevant case, the term $1+\left(\lambda / \lambda_{H}\right)^{2}$ can be replaced by unity. However, when the wavelength $\lambda$ of the perturbation approaches the cosmological horizon, this term must be taken into account and it reduces the growth of the perturbations (it weakens the effective gravitational constant $\left.G_{\text {eff }}(k)=G /\left(1+k_{H}^{2} / k^{2}\right)\right)$. As a result, it sets a natural upper cutoff, of the order of the Hubble length, for the perturbations that can grow. We shall come back to this reduction factor in Sec. VIIG.

\section{EVOLUTION OF THE DENSITY CONTRAST IN THE MATTER ERA}

In this section, we study the evolution of the density contrast $\delta_{k}$ in the matter era. We assume that the matter era starts at $a_{i}=10^{-4}$, corresponding to the epoch of matter-radiation equality. This is just before the recombination epoch (matter-dominated era) starting at $a_{\text {rec }}=10^{-3}$ when the electrons recombine with the atomic nuclei, after their last dispersion with the photons, leaving an imprint of this interaction in the temperature fluctuations of the CMB.

\section{A. The equation for $\delta_{k}(a)$ in the matter era}

In the matter era, we can make the approximations $\epsilon_{b} \sim \rho_{b} c^{2}$ and $P_{b} \ll \epsilon_{b}$ (see Sec. VC). In that limit, $\rho_{b}$ coincides with the rest-mass density and the Friedmann equations 31, 32 and 33 reduce to

$$
\begin{gathered}
\frac{d \rho_{b}}{d t}+3 H \rho_{b}=0, \\
H^{2}=\left(\frac{\dot{a}}{a}\right)^{2}=\frac{8 \pi G}{3} \rho_{b}, \\
\frac{\ddot{a}}{a}=-\frac{4 \pi G}{3} \rho_{b} .
\end{gathered}
$$

They lead to the EdS solution $\rho_{b} \propto a^{-3}, a \propto t^{2 / 3}, H=$ $2 /(3 t)$ and $\rho_{b}=1 /\left(6 \pi G t^{2}\right)$. Using Eqs. 121)-123), we can transform Eq. 116 for $\delta_{k}(t)$ into an equation for

10 Using the Friedmann equation 32 , the Hubble length can be written as a sort of Jeans length $\lambda_{H}=2 \pi c / \sqrt{8 \pi G \epsilon_{b} / c^{2}} a$ in which the speed of sound is replaced by the speed of light. It can be interpreted as a maximum wavelength. 
$\delta_{k}(a)$ of the form

$$
\begin{array}{r}
\frac{d^{2} \delta_{k}}{d a^{2}}+\frac{3}{2 a} \frac{d \delta_{k}}{d a}+\frac{3}{2 a^{2}}\left[\frac{\hbar^{2} k^{4}}{16 \pi G \rho_{b} m^{2} a^{4}}+\frac{c_{s}^{2}}{4 \pi G \rho_{b} a^{2}} k^{2}\right. \\
\left.-\frac{1}{1+\frac{3 H^{2} a^{2}}{k^{2} c^{2}}}\left(1+\frac{2 c_{s}^{2}}{c^{2}}\right)\left(1+\frac{3 c_{s}^{2}}{c^{2}}+\frac{\hbar^{2} k^{2}}{4 m^{2} c^{2} a^{2}}\right)\right] \delta_{k}=0,
\end{array}
$$

where we recall that $c_{s}^{2}$ is given by Eq. (59). Eq. (124) involves the following characteristic wavenumbers. The quantum Jeans wavenumber is defined by

$$
k_{Q}=\left(\frac{16 \pi G \rho_{b} m^{2} a^{4}}{\hbar^{2}}\right)^{1 / 4} .
$$

It can be written as $k_{Q}=\kappa_{Q} a^{1 / 4}$ with $\kappa_{Q}=$ $\left(16 \pi G \rho_{b} a^{3} m^{2} / \hbar^{2}\right)^{1 / 4}$. The self-interaction Jeans wavenumber is defined by

$$
k_{J}=\left(\frac{4 \pi G \rho_{b} a^{2}}{c_{s}^{2}}\right)^{1 / 2}=\left(\frac{G m^{3} a^{2}}{a_{s} \hbar^{2}}\right)^{1 / 2} .
$$

It can be written as $k_{J}=\kappa_{J} a$ with $\kappa_{J}=\left(G m^{3} / a_{s} \hbar^{2}\right)^{1 / 2}$. The Compton wavenumber is defined by

$$
k_{C}=\frac{2 m c a}{\hbar} .
$$

It can be written as $k_{C}=\kappa_{C} a$ with $\kappa_{C}=2 m c / \hbar$. The Hubble (or horizon) wavenumber is defined by

$$
k_{H}=\frac{\sqrt{3} H a}{c}=\left(\frac{8 \pi G \rho_{b} a^{2}}{c^{2}}\right)^{1 / 2} .
$$

It can be written as $k_{H}=\kappa_{H} / \sqrt{a}$ with $\kappa_{H}=$ $\left(8 \pi G \rho_{b} a^{3} / c^{2}\right)^{1 / 2}$. We also introduce the dimensionless parameter

$$
\sigma=\frac{4 \pi a_{s} \hbar^{2} \rho_{b} a^{3}}{m^{3} c^{2}}
$$

so that $c_{s}^{2} / c^{2}=\sigma / a^{3}$. In term of these parameters, Eq. (124) can be rewritten as (we omit the subscript $k$ on $\left.\delta_{k}\right)$ :

$$
\begin{aligned}
\frac{d^{2} \delta}{d a^{2}} & +\frac{3}{2 a} \frac{d \delta}{d a}+\frac{3}{2 a^{2}}\left[\frac{k^{4}}{\kappa_{Q}^{4} a}+\frac{k^{2}}{\kappa_{J}^{2} a^{2}}\right. \\
& \left.-\frac{1}{1+\frac{\kappa_{H}^{2}}{k^{2} a}}\left(1+\frac{2 \sigma}{a^{3}}\right)\left(1+\frac{3 \sigma}{a^{3}}+\frac{k^{2}}{\kappa_{C}^{2} a^{2}}\right)\right] \delta=0
\end{aligned}
$$

It is interesting to see how the different characteristic scales $\kappa_{Q}, \kappa_{J}, \kappa_{H}$ and $\kappa_{C}$ naturally appear in this equation.

\section{B. Justification of the nonrelativistic limit}

In this section, and in Secs. VIIC/VIIF we consider the case where the term associated with the Hubble length is very small: $\kappa_{H}^{2} / k^{2} a \ll 1$. For a given wavenumber $k$, this corresponds to a scale factor $a \gg \kappa_{H}^{2} / k^{2}$ and, for a given scale factor $a$, this corresponds to a wavenumber $k \gg \kappa_{H} / a^{1 / 2}$. Therefore, this approximation is valid when the wavelength of the perturbation is much smaller than the cosmological horizon on the timescale considered: $\lambda \ll \lambda_{H}(a)$.

A direct (non-trivial) consequence of this assumption is that the term $k^{4} / \kappa_{Q}^{4} a$ associated with the quantum Jeans length is always much larger than the term $k^{2} / \kappa_{C}^{2} a^{2}$ associated with the Compton length. Indeed, the condition $k^{4} / \kappa_{Q}^{4} a \gg k^{2} / \kappa_{C}^{2} a^{2}$ is equivalent to $a \gg \kappa_{Q}^{4} / k^{2} \kappa_{C}^{2}$. Now, from Eqs. 125, 127) and (128) we can easily establish the identities

$$
\left(k_{C} k_{H}\right)^{2}=2 k_{Q}^{4} \quad \text { and } \quad\left(\kappa_{C} \kappa_{H}\right)^{2}=2 \kappa_{Q}^{4} .
$$

As a result, $\kappa_{Q}^{4} / k^{2} \kappa_{C}^{2}=\kappa_{H}^{2} / 2 k^{2}<\kappa_{H}^{2} / k^{2}$. Therefore, the condition $a \gg \kappa_{H}^{2} / k^{2}$ automatically implies $a \gg \kappa_{Q}^{4} / k^{2} \kappa_{C}^{2}$ which itself implies $k^{4} / \kappa_{Q}^{4} a \gg k^{2} / \kappa_{C}^{2} a^{2}$. We stress that this inequality is independent on the mass $m$ of the bosons.

On the other hand, the condition $\sigma / a^{3} \ll 1$ will be satisfied during the whole matter era if it is satisfied initially, i.e. if $\sigma \ll a_{i}^{3}=10^{-12}$. This condition corresponds to $c_{s} \ll c$. In Appendix $\mathrm{G}$ we show that this condition is always satisfied in the matter era.

In conclusion, if we assume that the wavelength of the perturbation is always much smaller than the cosmological horizon during the matter era $\left(\lambda \ll \lambda_{H}(a)\right)$, and if we use the fact that $\sigma / a^{3} \ll 1$, Eq. 130 reduces to

$$
\frac{d^{2} \delta}{d a^{2}}+\frac{3}{2 a} \frac{d \delta}{d a}+\frac{3}{2 a^{2}}\left(\frac{k^{4}}{\kappa_{Q}^{4} a}+\frac{k^{2}}{\kappa_{J}^{2} a^{2}}-1\right) \delta=0,
$$

which is the nonrelativistic equation for the density contrast of a SF/BEC [97, 100]. Therefore, the nonrelativistic limit is valid in the matter era when $\lambda \ll \lambda_{H}(a)$.

The CDM model is recovered by taking $\kappa_{Q} \rightarrow+\infty$ and $\kappa_{J} \rightarrow+\infty$ in Eq. 132 yielding

$$
\frac{d^{2} \delta_{C D M}}{d a^{2}}+\frac{3}{2 a} \frac{d \delta_{C D M}}{d a}-\frac{3}{2 a^{2}} \delta_{C D M}=0 .
$$

The growing solution is $\delta_{C D M} \propto a$. It is usually considered that $\delta_{i} \sim 10^{-5}$ at the initial time $a_{i}=10^{-4}$ of matter-radiation equality [3. Therefore, the growing evolution of the density contrast in the CDM model can be written as

$$
\delta_{C D M}(a)=\frac{\delta_{i}}{a_{i}} a .
$$

We will take this CDM result as a reference, and compare it with the prediction of the SF model. We note that 
Eq. 132 for the density contrast of the SF reduces to Eq. (133) when $k \rightarrow 0$ and when $a \rightarrow+\infty$ because the quantum term $k^{4} / \kappa_{Q}^{4} a$ and the self-interaction term $k^{2} / \kappa_{J}^{2} a^{2}$ become negligible in these limits. Therefore, the $\mathrm{SF}$ is expected to behave similarly to CDM at large scales and at late times.

\section{Non-interacting scalar field}

For a noninteracting $\mathrm{SF}\left(\kappa_{J} \rightarrow+\infty\right)$, the equation determining the evolution of the density contrast reduces to

$$
\frac{d^{2} \delta}{d a^{2}}+\frac{3}{2 a} \frac{d \delta}{d a}+\frac{3}{2 a^{2}}\left(\frac{k^{4}}{\kappa_{Q}^{4} a}-1\right) \delta=0 .
$$

It turns out that Eq. 135 can be solved analytically [100. Its growing solution is given by

$$
\delta(a)=\frac{A}{a^{1 / 4}} J_{-\frac{5}{2}}\left(\sqrt{6} \frac{k^{2}}{\kappa_{Q}^{2}} \frac{1}{a^{1 / 2}}\right) .
$$

We shall determine the amplitude $A$ so that the asymptotic behavior of the density contrast of the SF at late times exactly matches the solution 134 of the CDM model. Using the asymptotic expansion of the Bessel function for small arguments (see, e.g., Appendix A of [100]), we obtain

$$
A=\frac{6^{5 / 4}}{3} \sqrt{\frac{\pi}{2}} \frac{\delta_{i}}{a_{i}}\left(\frac{k}{\kappa_{Q}}\right)^{5} .
$$

Eq. 136 with $A$ given by Eq. 137 determines the evolution of the density contrast of a non-interacting SF during the matter era in the nonrelativistic limit $\lambda \ll$ $\lambda_{H}(a)$.

We can identify two regimes. We first consider the case $k^{4} / \kappa_{Q}^{4} a \gg 1$. For a given wavenumber $k$, this corresponds to a scale factor $a \ll k^{4} / \kappa_{Q}^{4}$. Alternatively, for a given scale factor $a$, this corresponds to a wavelength $\lambda \ll \lambda_{Q}(a)$. Since the wavelength of the perturbation is smaller that the quantum Jeans length, the density contrast $\delta(a)$ displays oscillations. Using the asymptotic expansion of the Bessel function for small arguments, we find that

$$
\delta(a) \sim-2 \frac{\delta_{i}}{a_{i}}\left(\frac{k}{\kappa_{Q}}\right)^{4} \sin \left(\sqrt{6} \frac{k^{2}}{\kappa_{Q}^{2}} \frac{1}{a^{1 / 2}}\right) .
$$

This solution is valid for $a \ll k^{4} / \kappa_{Q}^{4}$. We see that the amplitude of the oscillations is constant, i.e. independent on the scale factor. We now consider the case $k^{4} / \kappa_{Q}^{4} a \ll 1$. For a given wavenumber $k$, this corresponds to a scale factor $a \gg k^{4} / \kappa_{Q}^{4}$. Alternatively, for a given scale factor $a$, this corresponds to a wavelength $\lambda \gg \lambda_{Q}(a)$. Since the wavelength of the perturbation is larger that the quantum Jeans length, the density contrast $\delta(a)$ increases. For $a \gg k^{4} / \kappa_{Q}^{4}$, the perturbation behaves like in the CDM model, see Eq. (134).

In summary, when $a \ll k^{4} / \kappa_{Q}^{4}$, i.e. $\lambda \ll \lambda_{Q}(a)$, the perturbation oscillates with a constant amplitude and when $a \gg k^{4} / \kappa_{Q}^{4}$, i.e. $\lambda \gg \lambda_{Q}(a)$, the perturbation grows linearly with the scale factor like in the CDM model.

To fix the ideas, we consider that the matter era starts at $a_{i}=10^{-4}$ and we study the evolution of the density contrast up to $a_{0}=1$, corresponding to the present-day universe (of course, our linear analysis is not valid up to that point, but our aim is just to give a simple illustration of our results). Our nonrelativistic treatment is valid on this interval provided that $a_{i} \gg \kappa_{H}^{2} / k^{2}$. This corresponds to wavenumbers $k \gg \kappa_{H} / a_{i}^{1 / 2}$. This constraint is not restrictive since $\kappa_{H} / a_{i}^{1 / 2}=6.49 \times 10^{-25} \mathrm{~m}^{-1}$ is very small (see Table I in Appendix F. For $k / \kappa_{Q} \geq a_{0}^{1 / 4}=1$, the evolution of the density contrast is purely oscillatory (see Fig. 22. For $k / \kappa_{Q} \leq a_{i}^{1 / 4}=0.1$, there is no oscillation and the density contrast grows from the beginning (see Fig. 33. ${ }^{11}$ In the intermediate situation $a_{i}^{1 / 4}=0.1<k / \kappa_{Q}<a_{0}^{1 / 4}=1$, the density contrast first presents oscillations until $a \sim k^{4} / \kappa_{Q}^{4}$, then grows (see Fig. 4). We note that the density contrast in the SF model grows faster than in the CDM model.

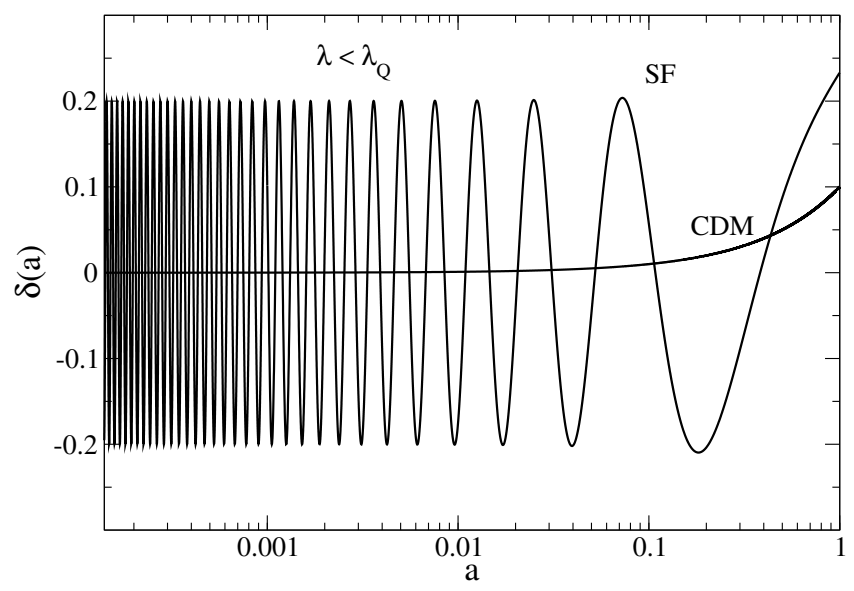

FIG. 2: Evolution of the density contrast $\delta(a)$ of a noninteracting SF for $k / \kappa_{Q}=1$ (semi-log plot). It is compared with the density contrast $\delta_{C D M}(a)$ of the CDM model.

The SF has several virtues. First, it does not create an over-abundance of small-scale structures, contrary to the CDM model, because the perturbations below the quan-

11 We can give a more general criterion for the absence of oscillations, independent on the interval of time considered. Our nonrelativistic treatment requires that $a \gg \kappa_{H}^{2} / k^{2}$ and the density contrast displays oscillations provided that $a \ll k^{4} / \kappa_{Q}^{4}$. Therefore, the density contrast will not display oscillations if $k \ll \kappa_{Q}^{2 / 3} \kappa_{H}^{1 / 3}$. 


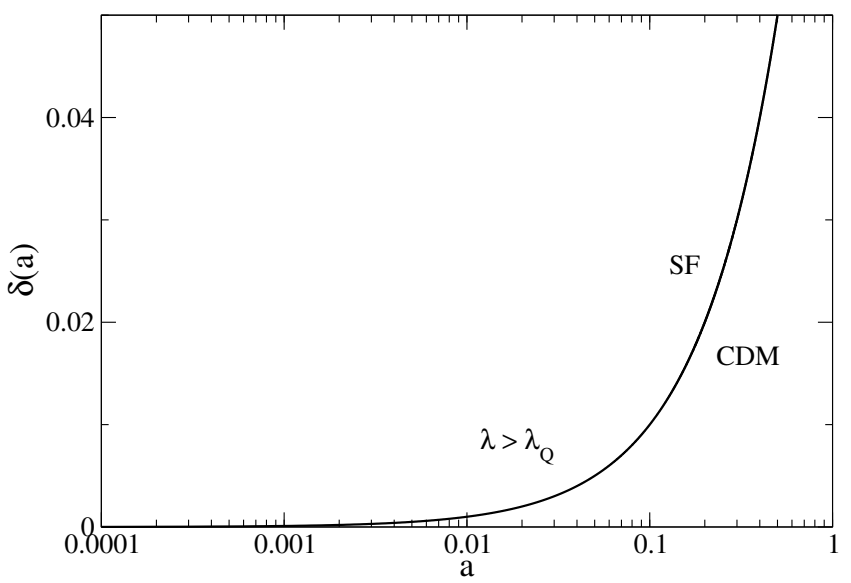

FIG. 3: Evolution of the density contrast $\delta(a)$ of a noninteracting SF for $k / \kappa_{Q}=0.1$ (semi-log plot).

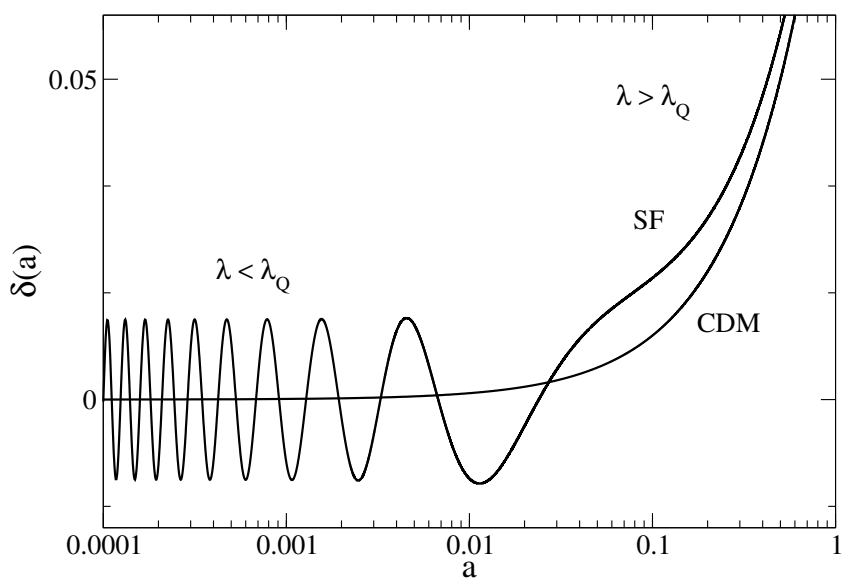

FIG. 4: Evolution of the density contrast $\delta(a)$ of a noninteracting $\mathrm{SF}$ for $k / \kappa_{Q}=0.5$ (semi-log plot).

tum Jeans length $\lambda_{Q}$ do not grow (they oscillate). The quantum Jeans length provides therefore a sharp smallscale cut-off in the matter power spectrum. On the other hand, the perturbations above the quantum Jeans length $\lambda_{Q}$ grow sensibly more rapidly than in the CDM model. This can accelerate the formation of large-scale structures in the nonlinear regime in agreement with some observations [119].

\section{Self-interacting scalar field in the Thomas-Fermi limit}

We now consider a self-interacting SF. We make the Thomas-Fermi (TF) approximation $\left(\kappa_{Q} \rightarrow+\infty\right)$ which amounts to neglecting the quantum term in front of the self-interaction term. In that case, the equation determining the evolution of the density contrast reduces to

$$
\frac{d^{2} \delta}{d a^{2}}+\frac{3}{2 a} \frac{d \delta}{d a}+\frac{3}{2 a^{2}}\left(\frac{k^{2}}{\kappa_{J}^{2} a^{2}}-1\right) \delta=0 .
$$

This equation can be solved analytically [100]. The growing solution is given by

$$
\delta(a)=\frac{B}{a^{1 / 4}} J_{-\frac{5}{4}}\left(\sqrt{\frac{3}{2}} \frac{k}{\kappa_{J}} \frac{1}{a}\right) .
$$

The amplitude $B$ is determined by requiring that the asymptotic behavior of Eq. 140 for $a \rightarrow+\infty$ exactly matches the solution (134) of the CDM model. This gives

$$
B=\Gamma\left(-\frac{1}{4}\right) \frac{3^{5 / 8}}{2^{15 / 8}}\left(\frac{k}{\kappa_{J}}\right)^{5 / 4} \frac{\delta_{i}}{a_{i}} .
$$

Eq. 140 with $B$ given by Eq. 141) determines the evolution of the density contrast of a self-interacting SF during the matter era in the nonrelativistic limit $\lambda \ll$ $\lambda_{H}(a)$ and in the TF limit.

We can identify two regimes. We first consider the case $k / \kappa_{J} a \gg 1$. For a given wavenumber $k$, this corresponds to a scale factor $a \ll k / \kappa_{J}$. Alternatively, for a given scale factor $a$, this corresponds to a wavelength $\lambda \ll \lambda_{J}(a)$. Since the wavelength of the perturbation is smaller that the self-interaction Jeans length, the density contrast $\delta(a)$ displays oscillations. Using the asymptotic expansion of the Bessel function for small arguments, we find that

$$
\begin{aligned}
\delta(a) \sim \Gamma\left(-\frac{1}{4}\right) & \frac{3^{3 / 8}}{2^{9 / 8}} \frac{1}{\sqrt{\pi}} \frac{\delta_{i}}{a_{i}} a^{1 / 4}\left(\frac{k}{\kappa_{J}}\right)^{3 / 4} \\
& \times \cos \left(\sqrt{\frac{3}{2}} \frac{k}{\kappa_{J}} \frac{1}{a}+\frac{3 \pi}{8}\right) .
\end{aligned}
$$

This solution is valid for $a \ll k / \kappa_{J}$. We see that the amplitude of the oscillations grows as $a^{1 / 4}$. This is a difference with respect to the non-interacting SF for which the amplitude of the oscillations is constant (see Sec. VIIC). We now consider the case $k / \kappa_{J} a \ll 1$. For a given wavenumber $k$, this corresponds to a scale factor $a \gg k / \kappa_{J}$. Alternatively, for a given scale factor $a$, this corresponds to a wavelength $\lambda \gg \lambda_{J}(a)$. Since the wavelength of the perturbation is larger that the selfinteraction Jeans length, the density contrast $\delta(a)$ increases. For $a \gg k / \kappa_{J}$, the perturbation behaves like in the CDM model, see Eq. (134).

In summary, when $a \ll k / \kappa_{J}$, i.e. $\lambda \ll \lambda_{J}(a)$, the perturbation oscillates with a growing amplitude scaling as $a^{1 / 4}$ and when $a \gg k / \kappa_{J}$, i.e. $\lambda \gg \lambda_{J}(a)$, the perturbation grows linearly with the scale factor like in the CDM model.

As before, we consider that the matter era starts at $a_{i}=10^{-4}$ and we study the evolution of the density contrast up to $a_{0}=1$. Our nonrelativistic treatment is valid for $k \gg \kappa_{H} / a_{i}^{1 / 2}=6.49 \times 10^{-25} \mathrm{~m}^{-1}$. For $k / \kappa_{J} \geq$ $a_{0}=1$, the evolution of the density contrast is purely oscillatory (see Fig. 5). For $k / \kappa_{J} \leq a_{i}=10^{-4}$, there is no oscillation and the density contrast grows from the beginning (the evolution is similar to the one represented 
in Fig. 3. ${ }^{12}$ In the intermediate situation $a_{i}=10^{-4}<$ $k / \kappa_{J}<1$, the density contrast first presents growing oscillations until $a \sim k / \kappa_{J}$, then grows linearly with $a$ (see Fig. 6). We note that the density contrast in the SF model grows faster than in the CDM model.

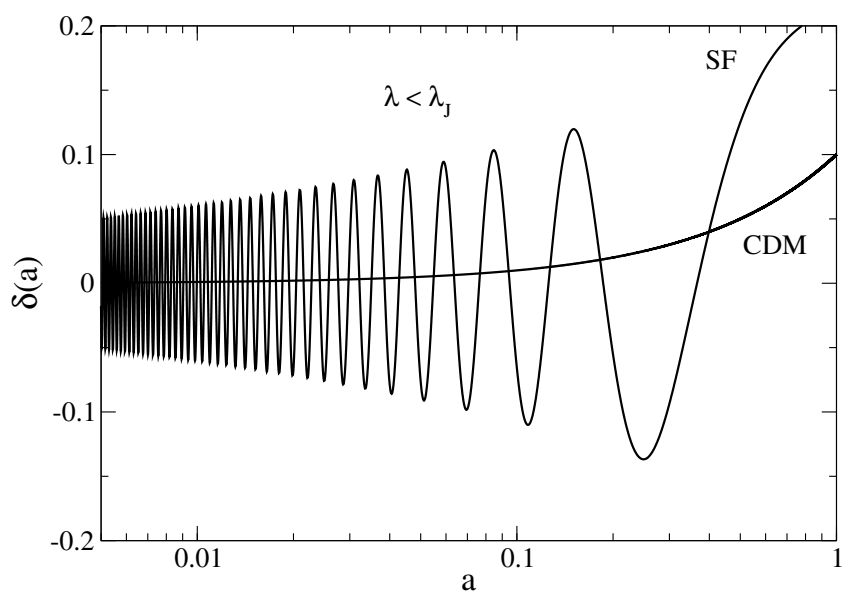

FIG. 5: Evolution of the density contrast $\delta(a)$ of a selfinteracting $\mathrm{SF}$ in the TF limit for $k / \kappa_{J}=1$ (semi-log plot).

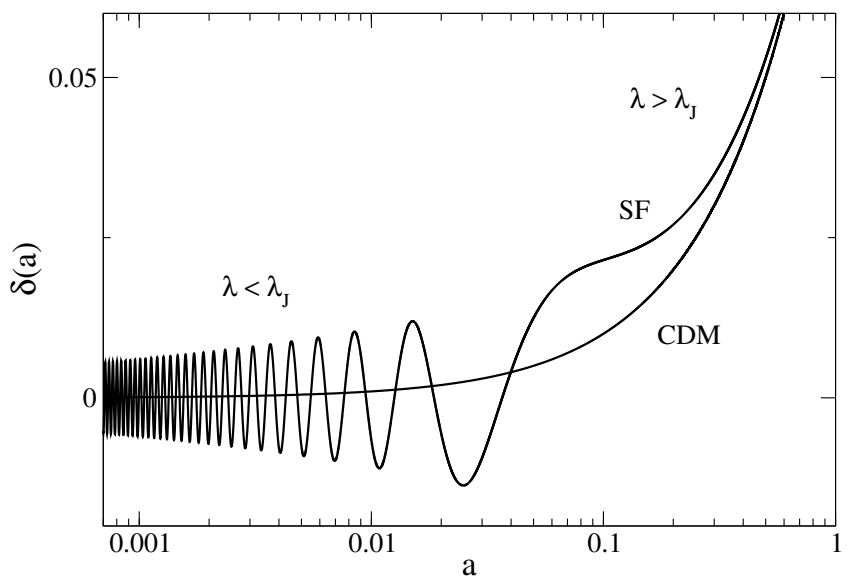

FIG. 6: Evolution of the density contrast $\delta(a)$ of a selfinteracting $\mathrm{SF}$ in the $\mathrm{TF}$ limit for $k / \kappa_{J}=0.1$ (semi-log plot).

The remarks concerning the virtues of the noninteracting SF made at the end of Sec. VIIC can be transposed to the self-interacting SF with the selfinteraction Jeans length replacing the quantum Jeans length. The SF suppresses small-scale structures below the self-interaction Jeans length $\lambda_{J}$ and accelerates the formation of structures above the self-interaction Jeans

12 The more general criterion for the absence of oscillations, similar to the one obtained in footnote 11 , is $k \ll \kappa_{J}^{1 / 3} \kappa_{H}^{2 / 3}$. length $\lambda_{J}$. This is an advantage of the SF model with respect to the CDM model. Note that the self-interaction Jeans length, which provides a sharp small-scale cut-off in the matter power spectrum, corresponds precisely to the size of the dark matter halos (see Eq. (G2) that form in the nonlinear regime [57, 67].

Remark: we note that the Compton term in Eq. 130 scales as $-k^{2} / \kappa_{C}^{2} a^{2}$ similarly to the self-interaction Jeans term $k^{2} / \kappa_{J}^{2} a^{2}$. Therefore, Eq. (139) with the Compton term retained can still be solved analytically by simply replacing $1 / \kappa_{J}^{2}$ by $1 / \kappa_{J}^{2}-1 / \kappa_{C}^{2}$. However, we have shown in Sec. VIIB (see also Sec. VII G) that the Compton term is always negligible in the matter era.

\section{E. Self-interacting scalar field beyond the TF limit}

We still consider a self-interacting SF but we now take the quantum term into account. The evolution of the density contrast is determined by Eq. 132. This equation has to be solved numerically. We would like to compare the solution of Eq. 132 with the solution 134 of the CDM model by imposing that they coincide at late times. However, there is a difficulty. Since the solution of Eq. 132 may be strongly oscillating at early time, it is not convenient to specify the initial condition at $a_{i}$ and solve the equation forward in time. Such a procedure would be very sensitive to the initial condition and would not in general reproduce the CDM result at late times. ${ }^{13}$ Instead, we can specify the initial conditions at $a_{0}=1$ (today) and solve the differential equation backwards in time until $a_{i}=10^{-4}$. This procedure has already been used in the past 98. Therefore, we solve Eq. 132 numerically with the initial condition $\left(\delta_{0}, \dot{\delta}_{0}\right)$ obtained from the analytical solution determined by Eqs. 136 and (137) at $a_{0}=1$. For illustration, we have taken $k / \kappa_{Q}=0.5$ and $\kappa_{Q} / \kappa_{J}=0.1$ in Fig. 7

The evolution of the perturbation can be understood qualitatively by using simple scaling arguments. The self-interaction term dominates the quantum term in Eq. (132) when $k^{2} / \kappa_{J}^{2} a^{2} \gg k^{4} / \kappa_{Q}^{4} a$. For a given wavenumber $k$, this corresponds to a scale factor $a \ll \kappa_{Q}^{4} / \kappa_{J}^{2} k^{2}$. Alternatively, for a given scale factor $a$, this corresponds

13 This is a particularity of the SF. Because of the oscillations of $\delta(a)$ at early time, the evolution of the perturbations with small wavelengths $\lambda$ is very dependent on the initial values of $\left(\delta_{i}, \dot{\delta}_{i}\right)$ prescribed at $a_{i}$. We need very particular values of $\left(\delta_{i}, \dot{\delta}_{i}\right)$ in order to recover the results of the CDM model at later times. This implies that, in general, the evolution of the perturbations with small wavelengths is different in the SF and CDM models, even at late times. This is not a drawback of the SF model because we know that the CDM model presents discrepencies with observations at small scales and that the SF model has been introduced precisely to solve them (see the discussion at the end of Secs. VIIC and VIID. Of course, for sufficiently large wavelengths $\lambda$, the fine tuning problem disappears, and the $\mathrm{SF}$ and CDM models give the same results at late time. 
to a wavelength $\lambda \gg \lambda_{Q}^{2} / \lambda_{J}$. For $a \ll \kappa_{Q}^{4} / \kappa_{J}^{2} k^{2}$, the system is in the TF regime and for $a \gg \kappa_{Q}^{4} / \kappa_{J}^{2} k^{2}$ it is in the non-interacting regime. In the TF regime, the perturbation undergoes growing oscillations for $a \ll k / \kappa_{J}$ and grows linearly for $a \gg k / \kappa_{J}$. In the non-interacting regime, the perturbation undergoes oscillations with a constant amplitude for $a \ll k^{4} / \kappa_{Q}^{4}$ and grows linearly for $a \gg k^{4} / \kappa_{Q}^{4}$.

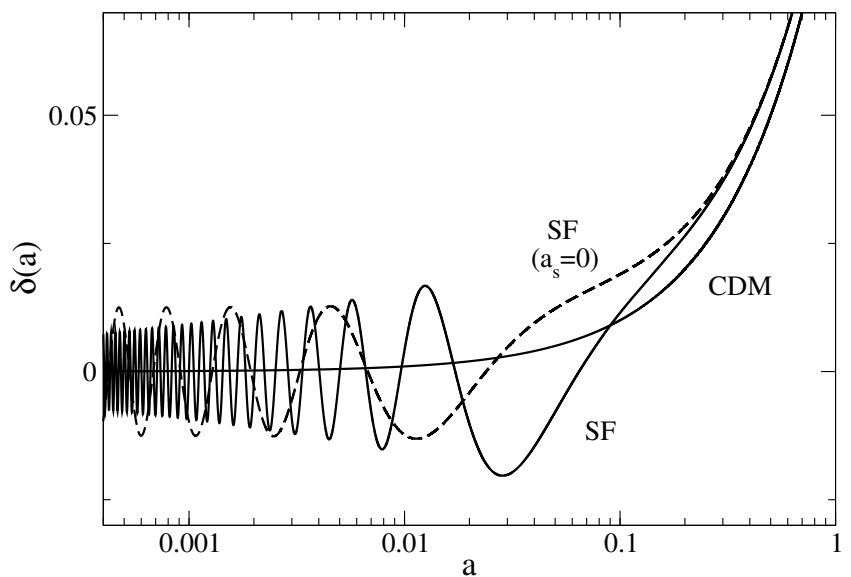

FIG. 7: Evolution of the density contrast $\delta(a)$ of a selfinteracting SF for $k / \kappa_{Q}=0.5$ and $\kappa_{Q} / \kappa_{J}=0.1$ (semi-log plot). It is compared with the non-interacting SF solution (dashed line).

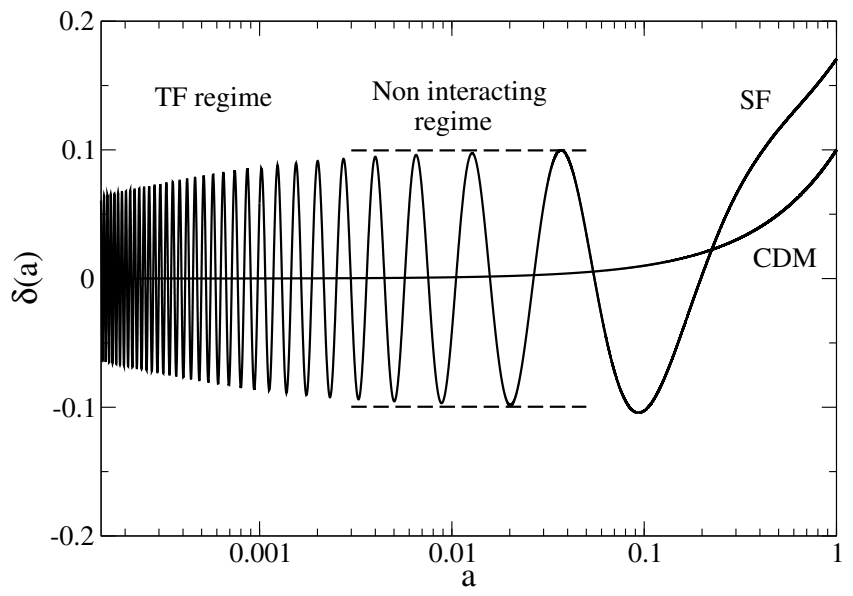

FIG. 8: Evolution of the density contrast $\delta(a)$ of a selfinteracting SF for $k / \kappa_{Q}=0.840$ and $\kappa_{Q} / \kappa_{J}=0.022$ (semi-log plot).

If $\kappa_{Q}^{4} / \kappa_{J}^{2} k^{2} \ll a_{i}$, the system is always in the noninteracting regime. If $\kappa_{Q}^{4} / \kappa_{J}^{2} k^{2} \gg a_{0}=1$, the system is always in the TF regime. In these cases, we are led back to the situations considered in Secs. VIIC and VIID, We now assume $a_{i}<\kappa_{Q}^{4} / \kappa_{J}^{2} k^{2}<1$. Two situations can occur.

(i) If $k / \kappa_{J}<\kappa_{Q}^{4} / \kappa_{J}^{2} k^{2}$, we necessarily have $k^{4} / \kappa_{Q}^{4}<$ $\kappa_{Q}^{4} / \kappa_{J}^{2} k^{2}$. Therefore, the perturbation undergoes grow- ing oscillations (provided that $k / \kappa_{J}>a_{i}$ ) followed by a linear growth. The growing oscillations take place at the begining of the TF regime. The linear growth starts at the end of the TF regime and continues in the noninteracting regime (there is no oscillation in the noninteracting regime because $\left.k^{4} / \kappa_{Q}^{4}<\kappa_{Q}^{4} / \kappa_{J}^{2} k^{2}\right)$. It is not possible to clearly see the change of regime during the linear growth so the evolution of the perturbation looks similar to that reported in Fig. 6 .

(ii) If $k^{4} / \kappa_{Q}^{4}>\kappa_{Q}^{4} / \kappa_{J}^{2} k^{2}$, we necessarily have $k / \kappa_{J}>$ $\kappa_{Q}^{4} / \kappa_{J}^{2} k^{2}$. In that case, the perturbations undergo growing oscillations, followed by constant-amplitude oscillations, and finally by a linear growth (provided that $\left.k^{4} / \kappa_{Q}^{4}<1\right)$. The growing oscillations take place in the TF regime. The constant-amplitude oscillations and the linear growth take place in the noninteracting regime (there is no linear growth in the $\mathrm{TF}$ regime because $\left.k / \kappa_{J}>\kappa_{Q}^{4} / \kappa_{J}^{2} k^{2}\right)$. In that case, the change of regime is clearly apparent since we can see the difference between growing oscillations and constant-amplitude oscillations. This is illustrated in Fig. 8

\section{F. The case of a negative scattering length}

The previous results implicitly assume that the scattering length is positive $\left(a_{s}>0\right)$, corresponding to a repulsive self-interaction. In this section, we consider the case of a negative scattering length $\left(a_{s}<0\right)$, corresponding to an attractive self-interaction. The equation for the density contrast becomes

$$
\frac{d^{2} \delta}{d a^{2}}+\frac{3}{2 a} \frac{d \delta}{d a}+\frac{3}{2 a^{2}}\left(\frac{k^{4}}{\kappa_{Q}^{4} a}-\frac{k^{2}}{\kappa_{J}^{2} a^{2}}-1\right) \delta=0,
$$

where now $\kappa_{J}=\left(G m^{3} /\left|a_{s}\right| \hbar^{2}\right)^{1 / 2}$. We first neglect the quantum term in Eq. 143) (TF approximation). In that case Eq. (143) reduces to

$$
\frac{d^{2} \delta}{d a^{2}}+\frac{3}{2 a} \frac{d \delta}{d a}+\frac{3}{2 a^{2}}\left(-\frac{k^{2}}{\kappa_{J}^{2} a^{2}}-1\right) \delta=0
$$

The growing solution of Eq. (144) is given by

$$
\delta(a) \propto \frac{1}{a^{1 / 4}} I_{-\frac{5}{4}}\left(\sqrt{\frac{3}{2}} \frac{k}{\kappa_{J}} \frac{1}{a}\right) .
$$

This solution diverges exponentially rapidly towards $-\infty$ when $a \rightarrow 0$. This divergence can be avoided by taking the decaying solution into account [100. Therefore, we solve Eq. (144) with the initial condition $\delta_{i}=10^{-5}$ and $\dot{\delta}_{i}=\delta_{i} / a_{i}=0.1$ at $a_{i}=10^{-4}$, obtained from Eq. (134). The result is represented in Fig. 9. For small $k$ (large scales), the density contrast evolves as in the CDM model. For large $k$ (small scales), the density contrast increases exponentially rapidly with the scale factor because the attraction due to the self-interaction adds 
to the gravitational attraction [100. An attractive selfinteraction therefore favors the growth of structures, but it does it in a rather dramatic manner. Indeed, it leads to an over-abundance of substructures at a very early epoch. This is in contradiction with the observations that do not show such an abundance of substructures. The CDM model, in which only gravity is in action, already presents too many substructures. Therefore, we need a mechanism that erases small-scale structures, not a mechanism that enhances them. Consequently, the SF should have in principle a positive scattering length, not a negative scattering length. ${ }^{14}$

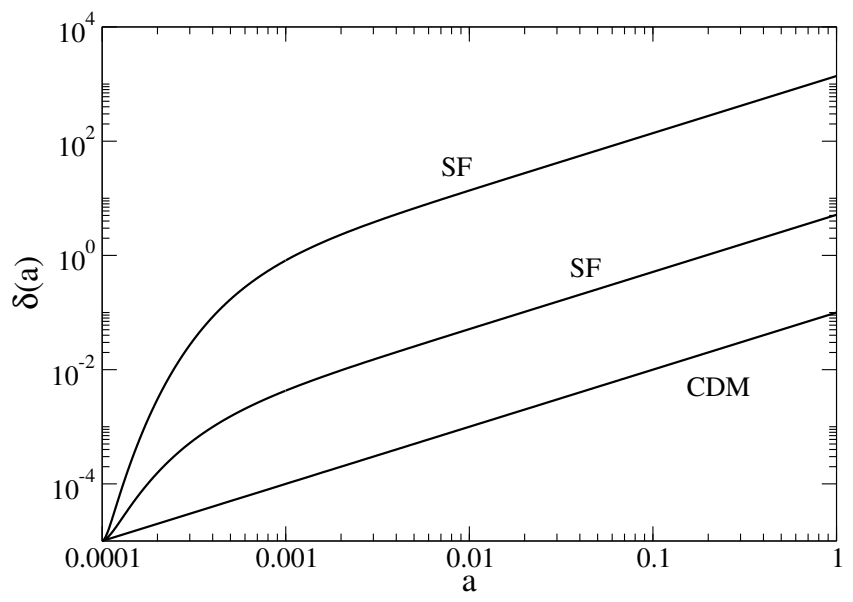

FIG. 9: Evolution of the density contrast $\delta(a)$ of a selfinteracting SF with $a_{s}<0$ in the TF limit for (bottom to top) $k / \kappa_{J}=0, k / \kappa_{J}=5 \times 10^{-4}$ and $k / \kappa_{J}=10^{-3}$ (log-log plot).

These conclusions are not true anymore if $\left|a_{s}\right|$ is extremely small (typically $\left|a_{s}\right| \ll 10^{-60} \mathrm{fm}$ ) because, in that case, we need to take into account the quantum term in Eq. (143) that stabilizes the structures at small scales. The quantum term becomes efficient when $a \gg \kappa_{Q}^{4} / \kappa_{J}^{2} k^{2}$. Therefore, it rapidly stabilizes the modes with large $k$ (small scales) and stops their growth. Indeed, after an exponential growth, the perturbation starts to oscillate (see Fig. 10). Modes with intermediate $k$ (intermediate scales) grow exponentially rapidly because the range where the attractive self-interaction dominates is longer (i.e. $\kappa_{Q}^{4} / \kappa_{J}^{2} k^{2}$ is larger). Finally, modes with small $k$ (large scales) grow slower (linearly with $a$ ) and behave like the CDM model. Therefore, bosons with an extremely small negative scattering length may be interesting DM candidates (as suggested in [100]) because they can accelerate the growth of perturbations, hence the formation of structures, at intermediate scales without

${ }^{14}$ In addition, it is shown in [57, 58] that it is not possible to form realistic dark matter halos when $a_{s}<0$ because they are unstable above a maximum mass $M_{\max }=1.012 \hbar / \sqrt{G m\left|a_{s}\right|}$ that is usually very small. forming too many substructures at small scales. These considerations may be relevant to QCD axions because they usually have an extremely small negative scattering length [52].

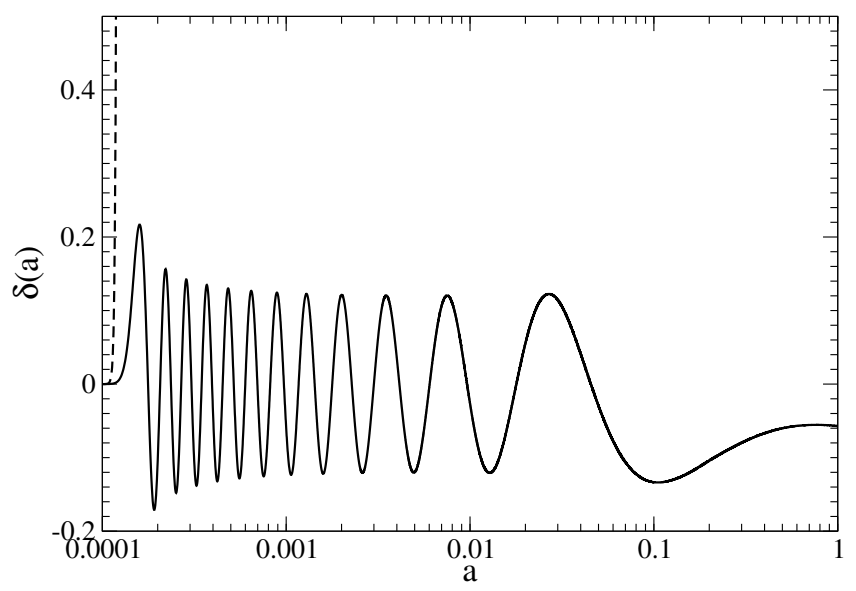

FIG. 10: Evolution of the density contrast $\delta(a)$ of a selfinteracting SF with $a_{s}<0$ for $\epsilon=\kappa_{Q} / \kappa_{J}=0.0085$ and $k / k_{Q}=0.7$ (semi-log plot). The dashed curve (exponential growth) represents the solution of Eq. 143 without the quantum term and the solid curve shows the stabilization due to the quantum term.

\section{G. Relativistic attenuation when $\lambda$ approaches the cosmological horizon}

We now consider the case where the wavelength $\lambda$ of the perturbation approaches the cosmological horizon $\lambda_{H}$ so that relativistic effects must be taken into account.

If we consider wavelengths $\lambda \sim \lambda_{H}$, or larger, then the Compton term $k^{2} / \kappa_{C}^{2} a^{2}$ in Eq. 130 is negligible in front of unity $\left(k^{2} / \kappa_{C}^{2} a^{2} \ll 1\right.$, i.e. $\left.\lambda \gg \lambda_{C}\right)$ because $\lambda_{H} \gg \lambda_{C}$ (see Appendix F). Since we have shown in Sec. VIIB that the Compton term is negligible in front of the quantum term when $\lambda \ll \lambda_{H}$, we conclude that the Compton term is always negligible in the matter era. We have also shown in Sec. VIIB that $\sigma / a^{3} \ll 1$ in the matter era. Therefore, the evolution of the density contrast in the matter era, valid for any perturbation, is determined by the equation

$$
\frac{d^{2} \delta}{d a^{2}}+\frac{3}{2 a} \frac{d \delta}{d a}+\frac{3}{2 a^{2}}\left(\frac{k^{4}}{\kappa_{Q}^{4} a}+\frac{k^{2}}{\kappa_{J}^{2} a^{2}}-\frac{1}{1+\frac{\kappa_{H}^{2}}{k^{2} a}}\right) \delta=0 .
$$

In the matter era, we have $\lambda_{C} \ll\left(\lambda_{Q}, \lambda_{J}\right) \ll \lambda_{H}$ (see Appendix F). For $\lambda \ll\left(\lambda_{Q}, \lambda_{J}\right)$, we can use the nonrelativistic results of Secs. VII C.VIIE since $\lambda \ll \lambda_{H}$. In that case, we have seen that the perturbation oscillates because the wavelength is smaller than the Jeans length. We now assume $\lambda \gg\left(\lambda_{Q}, \lambda_{J}\right)$. In that case, Eq. (146) 


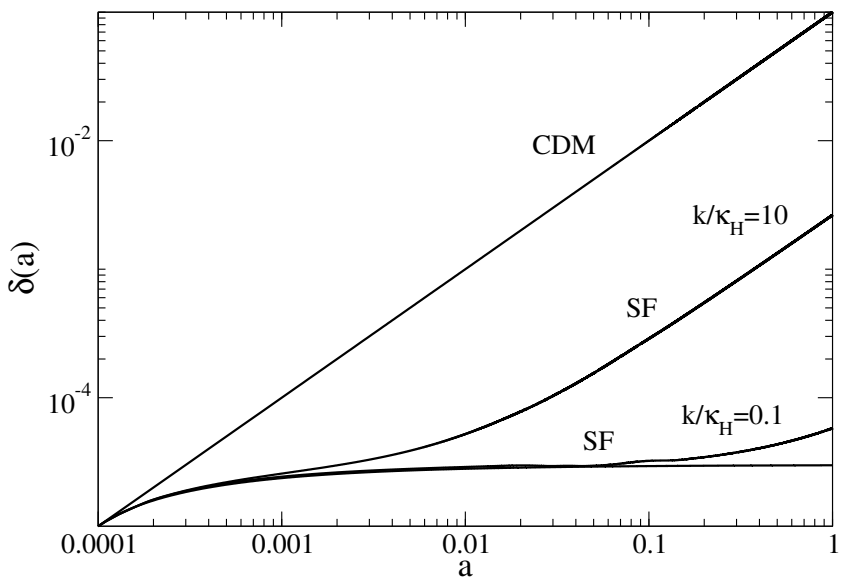

FIG. 11: Evolution of the density contrast $\delta(a)$ of a selfinteracting $\mathrm{SF}$ for $k / \kappa_{H}=10$ and $k / \kappa_{H}=0.1$ (log-log plot). We have also plotted the CDM solution (recovered for $k \gg \kappa_{H} / \sqrt{a}$ ) and the asymptotic solution $\sqrt{149}$ valid for $k \rightarrow 0$.

can be approximated by

$$
\frac{d^{2} \delta}{d a^{2}}+\frac{3}{2 a} \frac{d \delta}{d a}-\frac{3}{2 a^{2}} \frac{1}{1+\frac{\kappa_{H}^{2}}{k^{2} a}} \delta=0
$$

We solve Eq. 147 with the initial condition $\delta_{i}=10^{-5}$ and $\dot{\delta}_{i}=\delta_{i} / a_{i}=0.1$ at $a_{i}=10^{-4}$, obtained from Eq. (134). The last term in Eq. 147 corresponds to the gravitational attraction. For $\lambda \ll \lambda_{H}$, we can use the results of Secs. VIIC.VIIE and we find that the perturbation grows linearly with $a$, exactly like in the CDM model. Indeed, the term $\kappa_{H}^{2} / k^{2} a$ can be neglected and Eq. 147 reduces to Eq. 133 which has the growing solution of Eq. (134). We now consider the situation where $\lambda$ approaches the Hubble length $\lambda_{H}$. In that case, the weight of the gravitational term is reduced because of relativistic effects and, consequently, the perturbations grow slower. This is illustrated in Fig. 11. When $\lambda \rightarrow+\infty$, the gravitational term becomes negligible and Eq. 147 reduces to

$$
\frac{d^{2} \delta}{d a^{2}}+\frac{3}{2 a} \frac{d \delta}{d a}=0
$$

It has the solution

$$
\delta(a)=\delta_{i}\left(3-2 \sqrt{\frac{a_{i}}{a}}\right)
$$

which tends to a constant $3 \delta_{i}$ when $a \rightarrow+\infty$. This shows that when the wavelength approaches or overcomes the cosmological horizon, the perturbations do not grow anymore. There is a attenuation of relativistic origin.

Coming back to the general equation (146) and summarizing our results, we have shown that the relativistic term $1+k_{H}^{2} / k^{2}$ sets a natural upper cutoff, of the order of the Hubble length $\lambda_{H}$, above which the perturbations are attenuated. On the other hand, the terms $k^{4} / k_{Q}^{4}$ and $k^{2} / k_{Q}^{2}$ set natural lower cutoffs, of the order of the Jeans length $\lambda_{Q}$ or $\lambda_{J}$, below which the perturbations oscillate. In between, the perturbations grow linearly with $a$ as in the CDM model. Therefore, the effect of the relativistic SF is to introduce sharp cut-offs in the matter power spectrum at small scales $\lambda_{Q}$ or $\lambda_{J}$ because of quantum mechanics and at large scales $\lambda_{H}$ because of general relativity.

\section{ABOUT THE IMPORTANCE OF RELATIVISTIC EFFECTS}

Before concluding this paper, we would like to discuss the importance, or non-importance, of relativistic effects in the SFDM model.

In the matter era, we have shown that relativistic effects can be neglected as long as the wavelength of the perturbations is much smaller than the cosmological horizon. This is the case for most perturbations of physical interest. Since there is a wide range of scales satisfying $\left(\lambda_{Q}, \lambda_{J}\right) \ll \lambda \ll \lambda_{H}$, the perturbations can grow during the matter era and lead to the formation of structures in the nonlinear regime. However, in the stiff matter era and in the radiation era, relativistic effects are important because the wavelength of the perturbations may more easily reach the horizon (which is smaller). Since general relativity tends to prevent the growth of perturbations when their wavelength approaches the horizon, this may explain why the perturbations do not grow in the radiation era (the Jeans length may be larger than the horizon). This interesting effect will be studied in detail in future works.

On the other hand, even if relativistic corrections are weak in the matter era, we may wonder whether their effect could be detected in an era of "precision cosmology" [120. In particular, it would be interesting to see if one can observe differences between the KGE equations considered in this paper and the heuristic KGP equations studied in the past, in which gravity is introduced by hand in the KG equations (see Appendix D.

Relativistic effects are important for other astrophysical systems described by SFs or BECs. We can mention, for example, the case of boson stars 932 and the case of microscopic quantum black holes made of BECs of gravitons stuck at a quantum critical point [37, 121, 122]. It has also been proposed 32 that, because of their superfluid core, neutron stars could be BEC stars. Indeed, the neutrons (fermions) could form Cooper pairs and behave as bosons of mass $2 m_{n}$. This idea may solve certain issues regarding the maximum mass of neutron stars. To be complete, we should also mention analog models of gravity in which condensed matter systems described by the GP equation or by the KG equation are used to simulate results of classical and quantum field theory in curved space-time [123-125]. One of the first models for experimentally simulating black hole (Hawking) evapora- 
tion was suggested by Unruh in 1981 [123. Fifteen years later, a sonic analog of black holes specifically using BECs was developed [126]. Since then, various configurations of relativistic BECs have been proposed to simulate dif-

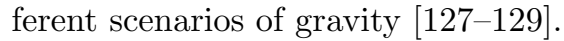

\section{CONCLUSION}

In this paper, we have developed a formalism based on a relativistic $\mathrm{SF}$ described by the KGE equations in the weak field limit. We have transformed these equations into equivalent hydrodynamic equations. These equations are arguably more tractable than the KGE equations themselves. In the nonrelativistic limit, they reduce to the hydrodynamic equations directly obtained from the GPP equations [100]. Therefore, our formalism clarifies the connection between the relativistic and nonrelativistic treatments. We note that, in the relativistic regime, the hydrodynamic variables $\psi, \rho, S, \vec{v}$, $p, \ldots$ that we have introduced do not have a direct physical interpretation. It is only in the nonrelativistic limit $c \rightarrow+\infty$ that they coincide with the wavefunction, restmass density, action, velocity, and pressure. However, these variables are perfectly well-defined mathematically from the $\mathrm{SF} \varphi$ in any regime, and they are totally legitimate. Furthermore, in terms of these variables, the relativistic hydrodynamic equations take a relatively simple form that provides a natural generalization of the nonrelativistic hydrodynamic equations. The main advantage in using the hydrodynamic representation of the SF is the facility of interpreting the results since the hydrodynamic equations are expressed in terms of familiar physical variables (density, velocity, pressure...). In addition, the use of these variables avoids the need of making averages over the oscillations of the SF as discussed in Sec. V This is valuable because it is not easy in practice to deal with the oscillations of the SF when directly solving the KGE equations numerically.

The complete study of the relativistic hydrodynamic equations is of considerable interest but it is, of course, of great complexity. In this paper, we have started their study in simple cases and we have obtained explicit results. Our paper shows therefore that these equations can be useful in practice.

First, we have considered the relativistic Jeans instability problem in a static universe. We have derived the relativistic dispersion relation and the relativistic Jeans length. Physical applications of these results will be developed in our companion papers.

Secondly, we have checked that the hydrodynamic equations of the SFDM model reproduce the evolution of the homogeneous background obtained previously by $\mathrm{Li}$ et al. 104 directly from the KGE equations: a stiff matter era, followed by a radiation era (for a self-interacting $\mathrm{SF}$ ), and a matter era.

Finally, we have started to analyze the evolution of the perturbations in an expanding universe dominated by a SF. We have considered the linear regime and we have focused on the matter era during which the growth of structures is expected to take place. In this regime, we can use the nonrelativistic limit of our formalism to treat the perturbations that are well inside the cosmological horizon $\left(\lambda \ll \lambda_{H}\right)$. We have evidenced analytically and numerically different regimes. Perturbations with $\lambda \ll \lambda_{J}$ oscillate with an amplitude growing as $a^{1 / 4}$, perturbations with $\lambda \ll \lambda_{Q}$ oscillate with a constant amplitude, perturbations with $\left(\lambda_{Q}, \lambda_{J}\right) \ll \lambda \ll \lambda_{H}$ grow linearly with $a$ like in the CDM model, and perturbations with $\lambda \sim \lambda_{H}$ are attenuated by general relativity and do not grow anymore. Therefore, the relativistic SF introduces sharp cut-offs in the matter power spectrum at small scales $\left(\lambda_{Q}\right.$ or $\left.\lambda_{J}\right)$ because of quantum mechanics and at large scales $\left(\lambda_{H}\right)$ because of general relativity.

Generically, we can use the TF approximation at early times, then the non-interacting approximation, and finally the classical (non quantum) approximation. As a result, the perturbations first display growing oscillations, followed by constant amplitude oscillations, and finally grow linearly with $a$ similarly to the CDM model (see Fig. 8). The growth of perturbations in the SF model is substantially faster than in the CDM model in agreement with certain cosmological observations where large-scale structures are observed at high redshifts 119. Depending on the wavelength of the perturbations, the duration of these different regimes in the matter-dominated era can change. We have provided all the scaling laws necessary to have a complete picture of the evolution of the perturbations in any case. In future works, we will extend our study of perturbations in the radiative era and in the stiff matter era where relativistic effects are expected to play an important role.

In this paper, we have studied the development of perturbations of the SFDM model in the linear regime. In future works, it will be important to consider the nonlinear regime where structure formation actually occurs. In general, this problem must be addressed numerically. The hydrodynamic equations derived in this paper may be very helpful because they may be easier to solve than the KGE equations. ${ }^{15}$ Therefore, numerical simulations using fluid dynamics should be developed in the future. As a first step, relativistic effects could be neglected and the nonrelativistic equations of Appendix C could be used. ${ }^{16}$ These equations are similar to the hydrodynamic equations of CDM except that they include a quantum

15 Since our equations are based on the Newtonian metric assuming that $\Phi / c^{2} \ll 1$, they cannot be extrapolated to the nonlinear relativistic regime where terms like $\Phi^{2} / c^{4}$ must be taken into account. However, our equations are exact in the linear relativistic regime which is relevant to describe the radiation era, and in the nonlinear nonrelativistic regime $(c \rightarrow+\infty)$ which is relevant to study the formation of structures in the matter era.

16 It may be mentioned that very nice numerical simulations have been made recently for nonrelativistic non-interacting SFDM/BECDM by directly solving the SP equations 60, 61. 
potential (Heisenberg) and a pressure term (scattering) that avoid singularities at small scales and may solve the missing satellite problem [100. In this respect, it may be recalled that the SP equations were introduced early by Widrow and Kaiser [130] as a procedure of small-scale regularization (a sort of mathematical trick) to prevent singularities in collisionless simulations of classical particles. In their approach, $\hbar$ is not the Planck constant, but rather an ajustable parameter that controls the spatial resolution. Their procedure finds a physical justification if DM is made of self-gravitating BECs [131.

In this paper, we have studied the evolution of a universe containing exclusively a SF representing dark matter. If we want to construct a more general cosmological model, we must take into account the contribution of other components such as normal ${ }^{17}$ radiation (due to relativistic particles: photons, neutrinos...), baryonic matter, dark energy (e.g. the cosmological constant), and possibly non-gravitational couplings (e.g. electromagnetism). Normal radiation, baryons, and the cosmological constant can be introduced straightforwardly in the energy-momentum tensor or in the Friedmann equations. Since our perturbation analysis is carried out during the matter-dominated era (dark matter), corresponding to $10^{-4}<a<1$, the contribution to the density contrast of the components different from the SF is subdominant, so these components are not expected to change the evolution of the perturbations significantly. In the radiation-dominated era, corresponding to $a<10^{-4}$, the growth of structures is inhibited because the velocity dispersion of the particles dominates their gravitational attraction (free streaming) and the wavelength of the perturbations that could trigger gravitational collapse lies outside the horizon making these perturbations ineffective. Non-trivial gravitational couplings must be treated specifically. For example, electromagnetism can be taken into account by adding the contribution of the Maxwell tensor in the Lagrangian. The hydrodynamic equations can then be generalized. In the case of a complex SF (charged), electromagnetic forces can have a significant influence on the formation of structures due to vortical motion.

We would like to conclude this paper by recalling the interest of the SFDM model with respect to the CDM model for what concerns astrophysical and cosmological observations. One of the main observations that can be related to our study is the recently observed large-scale structures at high redshifts 119. Contrary to the CDM model which does not seem to give sufficient time for such structures to form, SFDM perturbations seem to grow somewhat faster, thereby facilitating the formation of structures at earlier times, or more rapidly. Another

17 We call it "normal" in order to distinguish it from the radiation produced by a relativistic self-interacting $\mathrm{SF}$, which has a different physical origin (see 104 and Sec. VC. observation that can be related to our work is the "missing satellite problem" 132. Numerical simulations of CDM lead to an over-abundance of small structures because the Jeans length is equal to zero (or is very small). Such a large number of satellite galaxies is not observed in the Universe. By contrast, the SFDM model provides a natural cutoff for the formation of small structures, due to quantum mechanics, giving a possible solution to the missing satellite problem because the Jeans length is finite. Another problem of the CDM model is the "cuspcore problem". The CDM model predicts that DM halos should be cuspy (the central density should diverge as $r^{-1}$ ) [133. while observations reveal that they have a flat core [134. Once again, the quantum properties of the SFDM model may solve this problem. Indeed, the quantum potential arising from the Heisenberg uncertainty principle (for a noninteracting SF) or the pressure due to the scattering (for a self-interacting SF) prevent gravitational collapse at small scales and lead to central density cores instead of cusps. Other astrophysical and cosmological applications of our SFDM formalism will be given in future papers.

\section{Appendix A: The value of $A$}

The GP equation is obtained from the KG equation by means of the transformation

$$
\varphi=A e^{-i m c^{2} t / \hbar} \psi
$$

The constant $A$ can be computed as follows. Substituting Eq. (A1) in Eq. 21, we find that the energy density of the $\mathrm{SF}$ is given by

$$
\begin{aligned}
& \frac{\epsilon}{c^{2}}=\frac{T_{0}^{0}}{c^{2}}=\frac{1}{2}\left(1-\frac{2 \Phi}{c^{2}}\right) \frac{m^{2}}{\hbar^{2}} A^{2}|\psi|^{2}+\frac{m^{2}}{2 \hbar^{2}} A^{2}|\psi|^{2} \\
& +\frac{A^{2}}{2 c^{4}}\left(1-\frac{2 \Phi}{c^{2}}\right)\left|\frac{\partial \psi}{\partial t}\right|^{2}+\frac{A^{2}}{2 a^{2} c^{2}}\left(1+\frac{2 \Phi}{c^{2}}\right)|\nabla \psi|^{2} \\
& +\frac{2 \pi a_{s} m A^{4}}{\hbar^{2} c^{2}}|\psi|^{4}-\frac{m A^{2}}{\hbar c^{2}}\left(1-\frac{2 \Phi}{c^{2}}\right) \operatorname{Im}\left(\frac{\partial \psi}{\partial t} \psi^{*}\right) .
\end{aligned}
$$

Taking the nonrelativistic limit $c \rightarrow+\infty$ of this equation, we obtain

$$
\frac{\epsilon}{c^{2}} \rightarrow \frac{m^{2} A^{2}}{\hbar^{2}}|\psi|^{2}=\frac{m^{2} A^{2}}{\hbar^{2}} \rho,
$$

where $\rho=|\psi|^{2}$ is the rest-mass density. Since $\epsilon \sim \rho c^{2}$ in the nonrelativistic limit $c \rightarrow+\infty$, we find

$$
A=\frac{\hbar}{m} \text {. }
$$

\section{Appendix B: Spatially inhomogeneous clusters}

We consider a spatially inhomogeneous SF/BEC cluster at equilibrium, representing for example a dark matter star, a boson star, or a dark matter halo. This cluster 
results from the nonlinear development of the Jeans instability. It corresponds to a steady state of the hydrodynamic equations (41)- 44) with $a=1, H=0, \rho(\vec{x}, t)=$ $\rho(\vec{x}), \Phi(\vec{x}, t)=\Phi(\vec{x}), \vec{v}(\vec{x}, t)=\overrightarrow{0}$, and $S(\vec{x}, t)=S(t)$.

The equation of continuity (41) implies that $S=-E t$ where $E$ is a constant. Then, the Hamilton-Jacobi equation (42) and the Einstein equation (44) reduce to

$$
\begin{gathered}
\left(1+\frac{4 \Phi}{c^{2}}\right) \frac{\hbar^{2}}{2 m} \frac{\Delta \sqrt{\rho}}{\sqrt{\rho}}-m \Phi-\frac{4 \pi a_{s} \hbar^{2} \rho}{m^{2}}\left(1+\frac{2 \Phi}{c^{2}}\right) \\
+E\left(1+\frac{E}{2 m c^{2}}\right)=0, \\
\frac{\Delta \Phi}{4 \pi G}=\left(1-\frac{\Phi}{c^{2}}\right) \rho+\frac{E}{m c^{2}}\left(1+\frac{E}{2 m c^{2}}\right)\left(1-\frac{2 \Phi}{c^{2}}\right) \rho \\
+\frac{\hbar^{2}}{8 m^{2} c^{2}}\left(1+\frac{2 \Phi}{c^{2}}\right) \frac{(\vec{\nabla} \rho)^{2}}{\rho}+\frac{2 \pi a_{s} \hbar^{2}}{m^{3} c^{2}} \rho^{2} .
\end{gathered}
$$

These two coupled equations determine the equilibrium state of a spatially inhomogeneous relativistic selfgravitating $\mathrm{SF} / \mathrm{BEC}$. If we neglect the terms in $\Phi / c^{2}$ (see the simplified model of Appendix D, they reduce to

$$
\begin{gathered}
\frac{\hbar^{2}}{2 m} \frac{\Delta \sqrt{\rho}}{\sqrt{\rho}}-m \Phi-\frac{4 \pi a_{s} \hbar^{2} \rho}{m^{2}}+E\left(1+\frac{E}{2 m c^{2}}\right)=0 \\
\frac{\Delta \Phi}{4 \pi G}=\rho+\frac{E}{m c^{2}}\left(1+\frac{E}{2 m c^{2}}\right) \rho \\
+\frac{\hbar^{2}}{8 m^{2} c^{2}} \frac{(\vec{\nabla} \rho)^{2}}{\rho}+\frac{2 \pi a_{s} \hbar^{2}}{m^{3} c^{2}} \rho^{2}
\end{gathered}
$$

Taking the Laplacian of Eq. B3 and substituting the result in Eq. (B4), we obtain a single differential equation for the density

$$
\begin{array}{r}
\frac{\hbar^{2}}{8 \pi G m^{2}} \Delta\left(\frac{\Delta \sqrt{\rho}}{\sqrt{\rho}}\right)-\frac{a_{s} \hbar^{2}}{G m^{3}} \Delta \rho=\rho+\frac{\hbar^{2}}{8 m^{2} c^{2}} \frac{(\vec{\nabla} \rho)^{2}}{\rho} \\
+\frac{2 \pi a_{s} \hbar^{2}}{m^{3} c^{2}} \rho^{2}+\frac{E}{m c^{2}}\left(1+\frac{E}{2 m c^{2}}\right) \rho .
\end{array}
$$

In the nonrelativistic limit $c \rightarrow+\infty$, it reduces to

$$
\frac{\hbar^{2}}{2 m^{2}} \Delta\left(\frac{\Delta \sqrt{\rho}}{\sqrt{\rho}}\right)-\frac{4 \pi a_{s} \hbar^{2}}{m^{3}} \Delta \rho=4 \pi G \rho .
$$

This returns the differential equation describing the equilibrium state of a spatially inhomogeneous nonrelativistic self-gravitating BEC studied in [57, 58].

\section{Appendix C: The nonrelativistic limit}

In the nonrelativistic limit $c \rightarrow+\infty$, the evolution of the wave function $\psi(\vec{x}, t)$ in an expanding universe is determined by the GPP equations [100]:

$i \hbar \frac{\partial \psi}{\partial t}+\frac{3}{2} i \hbar H \psi=-\frac{\hbar^{2}}{2 m a^{2}} \Delta \psi+m \Phi \psi+\frac{4 \pi a_{s} \hbar^{2}}{m^{2}}|\psi|^{2} \psi$,

$$
\frac{\Delta \Phi}{4 \pi G a^{2}}=|\psi|^{2}-\frac{3 H^{2}}{8 \pi G}
$$

These equations are equivalent to the quantum EulerPoisson equations 100:

$$
\begin{gathered}
\frac{\partial \rho}{\partial t}+3 H \rho+\frac{1}{a} \vec{\nabla} \cdot(\rho \vec{v})=0 \\
\frac{\partial S}{\partial t}+\frac{(\nabla S)^{2}}{2 m a^{2}}=\frac{\hbar^{2}}{2 m a^{2}} \frac{\Delta \sqrt{\rho}}{\sqrt{\rho}}-m \Phi-\frac{4 \pi a_{s} \hbar^{2} \rho}{m^{2}} \\
\frac{\partial \vec{v}}{\partial t}+H \vec{v}+\frac{1}{a}(\vec{v} \cdot \vec{\nabla}) \vec{v}= \\
\frac{\hbar^{2}}{2 m^{2} a^{3}} \vec{\nabla}\left(\frac{\Delta \sqrt{\rho}}{\sqrt{\rho}}\right)-\frac{1}{a} \vec{\nabla} \Phi-\frac{1}{\rho a} \vec{\nabla} p \\
\frac{\Delta \Phi}{4 \pi G a^{2}}=\rho-\frac{3 H^{2}}{8 \pi G}
\end{gathered}
$$

where the pressure $p$ is given by Eq. 45. The Euler equation (C5) includes a quantum potential $Q=$ $-\hbar^{2} /\left(2 m a^{3}\right) \Delta(\sqrt{\rho}) / \sqrt{\rho}$ arising from the Heisenberg uncertainty principle. In the classical limit $\hbar \rightarrow 0$ (or in the TF limit), we recover the classical Euler-Poisson equations for a barotropic fluid [117].

For a spatially homogeneous SF/BEC, the hydrodynamic equations $\mathrm{C} 3 \mathrm{C}-\mathrm{C} 6 \mathrm{r}$ reduce to

$$
\begin{gathered}
\frac{d \rho_{b}}{d t}+3 H \rho_{b}=0, \\
E=-\frac{d S_{b}}{d t}=\frac{4 \pi a_{s} \hbar^{2} \rho_{b}}{m^{2}}, \\
\frac{3 H^{2}}{8 \pi G}=\rho_{b} .
\end{gathered}
$$

From Eq. $\mathrm{C} 7$, we find that $\rho_{b} \propto a^{-3}$, so the homogeneous SF/BEC behaves as CDM. Then, Eq. C9 implies $a \propto t^{2 / 3}$ and $\rho_{b}=1 /\left(6 \pi G t^{2}\right)$, which is the EdS solution. Combining these results with Eq. (C8), we find that the energy of the homogeneous SF decreases as

$$
E(t)=\frac{2 a_{s} \hbar^{2}}{3 G m^{2} t^{2}} .
$$

Accordingly, its phase decreases as $S_{b}(t)=$ $2 a_{s} \hbar^{2} / 3 G m^{2} t+C$. To our knowledge, these results have not been given before. The energy density and the pressure of the homogeneous SF are given by

$$
\epsilon_{b}=\rho_{b} c^{2}, \quad P_{b}=\frac{2 \pi a_{s} \hbar^{2}}{m^{3}} \rho_{b}^{2} .
$$

The case of a static Universe is recovered by taking $a=1$ and $H=0$ in the foregoing equations. For a static homogeneous SF, we find that $S_{b}(t)=-E t$ with

$$
E=\frac{4 \pi a_{s} \hbar^{2} \rho_{b}}{m^{2}}=m c_{s}^{2}
$$

The energy of the SF is given by a sort of Einstein equation " $E=m c^{2}$ " where the speed of light is replaced by the speed of sound. 


\section{Appendix D: Generalized Klein-Gordon-Poisson equations}

In this Appendix, we consider a simplified model in which we introduce the gravitational potential $\Phi(\vec{x}, t)$ in the ordinary KG equation by hand, as an external potential, and assume that this potential is produced by the SF itself via a generalized Poisson equation in which the source is the energy density $\epsilon$. This leads to the generalized KGP equations. We then show that these equations can be rigorously justified from the KGE equations in the limit $\Phi / c^{2} \rightarrow 0$. Finally, we argue that this simplified model is not sufficient to study the evolution of the perturbations in the linear relativistic regime.

\section{The FLRW metric and the phenomenological interaction Lagrangian}

We consider the FLRW metric that describes an isotropic and homogeneous expanding background. The line element in the comoving frame is

$$
d s^{2}=g_{\mu \nu} d x^{\mu} d x^{\nu}=c^{2} d t^{2}-a(t)^{2} \delta_{i j} d x^{i} d x^{j} .
$$

For this metric, the d'Alembertian operator (7) writes

$$
\square=\frac{1}{c^{2}} \frac{\partial^{2}}{\partial t^{2}}+\frac{3 H}{c^{2}} \frac{\partial}{\partial t}-\frac{1}{a^{2}} \Delta .
$$

In order to take the self-gravity of the SF into account, we introduce a Lagrangian of interaction that couples the gravitational potential $\Phi(\vec{x}, t)$ to the scalar field $\varphi(\vec{x}, t)$ according to

$$
\mathcal{L}_{\text {int }}=-\frac{m^{2}}{\hbar^{2}} \Phi|\varphi|^{2}
$$

The total Lagrangian of the system $(\mathrm{SF}+$ gravity) is given by $\mathcal{L}=\mathcal{L}_{\varphi}+\mathcal{L}_{\text {int }}$.

\section{The Klein-Gordon equation}

The equation of motion resulting from the stationarity of the total action $S=S_{\varphi}+S_{\text {int }}$, obtained by writing $\delta S=0$, is the KG equation

$$
\square \varphi+2 V\left(|\varphi|^{2}\right),_{\varphi^{*}}+\frac{2 m^{2}}{\hbar^{2}} \Phi \varphi=0,
$$

where the d'Alembertian operator is given by Eq. (D2) and the gravitational potential $\Phi(\vec{x}, t)$ acts here as an external potential. For the specific SF potential (3), we obtain

$$
\begin{array}{r}
\frac{1}{c^{2}} \frac{\partial^{2} \varphi}{\partial t^{2}}+\frac{3 H}{c^{2}} \frac{\partial \varphi}{\partial t}-\frac{1}{a^{2}} \Delta \varphi \\
+\left(1+\frac{2 \Phi}{c^{2}}\right) \frac{m^{2} c^{2}}{\hbar^{2}} \varphi+\frac{8 \pi a_{s} m}{\hbar^{2}}|\varphi|^{2} \varphi=0
\end{array}
$$

The energy density and the pressure, defined from the diagonal part of the energy-momentum tensor (13), are given by

$$
\begin{gathered}
\epsilon=\frac{1}{2 c^{2}}\left|\frac{\partial \varphi}{\partial t}\right|^{2}+\frac{1}{2 a^{2}}|\vec{\nabla} \varphi|^{2}+V\left(|\varphi|^{2}\right), \\
P=\frac{1}{2 c^{2}}\left|\frac{\partial \varphi}{\partial t}\right|^{2}-\frac{1}{6 a^{2}}|\vec{\nabla} \varphi|^{2}-V\left(|\varphi|^{2}\right) .
\end{gathered}
$$

\section{The generalized Poisson equation}

Eq. (D5) is the ordinary KG equation for a SF in an external potential $\Phi(\vec{x}, t)$ in an expanding background. We now state that $\Phi(\vec{x}, t)$ is actually the gravitational potential produced by the SF itself. We phenomenologically assume that the gravitational potential is determined by a generalized Poisson equation of the form

$$
\frac{\Delta \Phi}{4 \pi G a^{2}}=\frac{1}{c^{2}}\left(\epsilon-\epsilon_{b}\right)
$$

in which the source of the gravitational potential is the energy density $\epsilon$ of the SF (more precisely, its deviation from the homogeneous background density $\left.\epsilon_{b}(t)\right)$. Using Eq. (D6) for the energy density of a SF, and recalling the Friedmann equation (32), the generalized Poisson equation can be written as

$$
\begin{aligned}
\frac{\Delta \Phi}{4 \pi G a^{2}}=\frac{1}{2 c^{4}}\left|\frac{\partial \varphi}{\partial t}\right|^{2} & +\frac{1}{2 a^{2} c^{2}}|\vec{\nabla} \varphi|^{2}+\frac{m^{2}}{2 \hbar^{2}}|\varphi|^{2} \\
& +\frac{2 \pi a_{s} m}{\hbar^{2} c^{2}}|\varphi|^{4}-\frac{3 H^{2}}{8 \pi G}
\end{aligned}
$$

Eqs. (D5) and (D9) form the generalized KGP equations. They have been introduced in an ad hoc manner but they can be rigorously justified from the KGE equations 20 and 26 in the limit $\Phi / c^{2} \rightarrow 0$ (which, of course, is different from the nonrelativistic limit $c \rightarrow+\infty)$. We see that the gravitational potential $\Phi$ appears in the KG equation (D5) due to the cancelation of $c^{2}$ in the product $\Phi / c^{2} \times c^{2}$ in Eq. 20. Therefore, we do not have to introduce $\Phi$ by hand: the generalized KGP equations can be obtained from the KGE equations by simply neglecting terms of order $\Phi / c^{2}$ in these equations. Similarly, the equations related to the generalized KGP equations (e.g. the generalized GPP equations, the corresponding hydrodynamic equations...) can be obtained from the ones related to the KGE equations by neglecting terms of order $\Phi / c^{2}$. Therefore, we do not write them explicitly (they are given in [112]). Now, a warning is required. This model correctly describes the homogeneous background for which $\Phi=0$ but it is not sufficient to describe the evolution of the perturbations in the linear relativistic regime because we must precisely take into account the terms of order $\Phi / c^{2}$ in this regime (except, of course, in the nonrelativistic limit $c \rightarrow+\infty$ ). Therefore, the use of 
the KGE equations is mandatory to study the evolution of the perturbations in the relativistic regime. To show that the generalized KGP equations may give wrong results, we derive in the next two subsections the dispersion relation of the perturbations in a static universe (Jeans problem) and the equation for the density contrast in an expanding universe based on the KGP equations and compare their expressions with those obtained from the KGE equations.

Remark: We could also assume that the gravitational potential is determined by a Poisson equation of the form

$$
\frac{\Delta \Phi}{4 \pi G a^{2}}=\rho
$$

in which the source of the gravitational potential is the pseudo rest-mass density $\rho=|\psi|^{2}$ of the SF. Eqs. D5 and (D10) form the KGP equations. This approximation has been considered in 97. However, there is an inconsistency in coupling the relativistic KG equation (D5) to the classical Poisson equation (D10.

\section{Dispersion relation in a static universe}

We consider the linear evolution of small perturbations in a static universe. For the generalized KGP equations, Eqs. 66- (68) can be reduced to a system of two coupled wave equations

$$
\begin{gathered}
\frac{1}{c^{2}} \frac{\partial^{2} \sigma}{\partial t^{2}}-\Delta \sigma=\left(1+\frac{E}{m c^{2}}\right) \frac{\partial^{2} \delta}{\partial t^{2}}, \quad \text { (D11) } \\
\left(1+\frac{E}{m c^{2}}\right)\left(\Delta \sigma-\frac{4 \pi G \rho_{b}}{c^{2}} \sigma\right)=\frac{\hbar^{2}}{4 m^{2}} \Delta\left(\Delta \delta-\frac{1}{c^{2}} \frac{\partial^{2} \delta}{\partial t^{2}}\right) \\
-c_{s}^{2} \Delta \delta-4 \pi G \rho_{b}\left(1+\frac{c_{s}^{2}}{c^{2}}\right) \delta-4 \pi G \rho_{b} \frac{E}{m c^{2}}\left(1+\frac{E}{2 m c^{2}}\right) \delta .
\end{gathered}
$$

Decomposing the perturbations in Fourier modes, we obtain the dispersion relation

$$
\begin{array}{r}
\frac{\hbar^{2}}{4 m^{2} c^{4}} \omega^{4}-\left(1+\frac{3 c_{s}^{2}}{c^{2}}+\frac{\hbar^{2} k^{2}}{2 m^{2} c^{2}}\right) \omega^{2} \\
+\left[\frac{\hbar^{2} k^{4}}{4 m^{2}}+c_{s}^{2} k^{2}-4 \pi G \rho_{b}\left(1+\frac{2 c_{s}^{2}}{c^{2}}\right)\right]=0 .
\end{array}
$$

Taking $\omega=0$ in Eq. (D13), we find that the Jeans length is determined by the equation

$$
\frac{\hbar^{2} k_{J}^{4}}{4 m^{2}}+c_{s}^{2} k_{J}^{2}-4 \pi G \rho_{b}\left(1+\frac{2 c_{s}^{2}}{c^{2}}\right)=0 .
$$

These equations differ from Eqs. (69) and 72 obtained in Sec. IVD starting from the KGE equations.

\section{Approximate equation for the density contrast in an expanding universe}

We consider the evolution of small perturbations in an expanding universe. For the generalized KGP equations, Eqs. 110-113 can be reduced to a system of two coupled wave equations

$$
\begin{gathered}
\frac{\partial^{2} \delta}{\partial t^{2}}+2 H \frac{\partial \delta}{\partial t}=\frac{c_{s}^{2}}{a^{2}} \Delta \delta-\frac{\hbar^{2}}{4 m^{2} a^{4}} \Delta^{2} \delta+\frac{1}{a^{2}} \Delta \Phi \\
\frac{\Delta \Phi}{4 \pi G \rho_{b} a^{2}}=\left(1+2 \frac{c_{s}^{2}}{c^{2}}\right) \delta-\frac{\hbar^{2}}{4 m^{2} c^{2} a^{2}} \Delta \delta+\frac{\Phi}{c^{2}} \\
+\frac{E}{m c^{2}}\left(\frac{E}{2 m c^{2}}+1\right) \delta
\end{gathered}
$$

Decomposing the perturbations in (spatial) Fourier modes, we obtain a closed equation for the density contrast

$$
\begin{array}{r}
\frac{d^{2} \delta_{k}}{d t^{2}}+2 H \frac{d \delta_{k}}{d t}+\left[\frac{\hbar^{2} k^{4}}{4 m^{2} a^{4}}+\frac{c_{s}^{2}}{a^{2}} k^{2}\right. \\
\left.-\frac{4 \pi G \rho_{b}}{1+\frac{4 \pi G \rho_{b} a^{2}}{k^{2} c^{2}}}\left(1+\frac{3 c_{s}^{2}}{c^{2}}+\frac{\hbar^{2} k^{2}}{4 m^{2} c^{2} a^{2}}\right)\right] \delta_{k}=0 .
\end{array}
$$

In a static universe $(a=1, H=0)$, taking $\ddot{\delta}_{k}=0$ (i.e. $\omega=0$ ), we can check that Eq. (D17) reduces to Eq. (D14) that determines the relativistic Jeans length derived from the generalized KGP equations. However, Eq. (D17) differs from Eq. 116) obtained in Sec. VIB starting from the KGE equations.

\section{Appendix E: Typical mass and scattering length of bosonic particles}

\section{Dark matter halos}

We assume that dark matter halos are made of bosons in the form of BECs. We determine the mass of the bosons that compose dark matter halos according to whether they are noninteracting or self-interacting. For dark matter halos, we can use Newtonian gravity. The smallest known dark matter halo is Willman 1. It has a radius $R=33 \mathrm{pc}$ and a mass $M=0.3910^{6} M_{\odot}$ [135, 136]. We assume that this most compact halo is completely condensed, i.e. that it corresponds to the ground state $(T=0)$ of a self-gravitating Bose gas without radiative halo. ${ }^{18}$

\footnotetext{
18 For a value of the boson mass in the range $2.57 \times 10^{-20} \mathrm{eV} / c^{2}<$ $m<1.69 \times 10^{-2} \mathrm{eV} / c^{2}$ (see below), we have $T \ll T_{c}$ (where $T_{c}$ is the condensation temperature) for all the dark matter halos of the Universe (with mass $M \sim 10^{6}-10^{11} M_{\odot}$ ), so they can be
} 
A completely condensed system of self-gravitating bosons (BEC) without self-interaction at $T=0$ has the mass-radius relation $M R=9.95 \hbar^{2} /\left(G m^{2}\right)$ [10, 39, 58]. This gives

$$
\frac{m}{\mathrm{eV} / c^{2}}=9.2210^{-17}\left(\frac{\mathrm{pc}}{R}\right)^{1 / 2}\left(\frac{M_{\odot}}{M}\right)^{1 / 2} .
$$

Using the values of $M$ and $R$ corresponding to Willman 1 , we obtain a boson mass $m=2.5710^{-20} \mathrm{eV} / c^{2} .^{19}$

A completely condensed system of self-gravitating bosons (BEC) with self-interaction at $T=0$ in the $\mathrm{TF}$ limit has a unique radius $R=\pi\left(a_{s} \hbar^{2} / G m^{3}\right)^{1 / 2}$ [57, 64, 66, 67. This gives

$$
\left(\frac{\mathrm{fm}}{a_{s}}\right)^{1 / 3}\left(\frac{m}{\mathrm{eV} / c^{2}}\right)=6.73\left(\frac{\mathrm{pc}}{R}\right)^{2 / 3} .
$$

Using the value of $R$ corresponding to Willman 1 , we obtain $\left(\mathrm{fm} / a_{s}\right)^{1 / 3}\left(m c^{2} / \mathrm{eV}\right)=0.654$. In order to determine the mass of the bosons, we need another relation (see footnote 1 in [103]). This relation is provided by the constraint $\sigma / m<1.25 \mathrm{~cm}^{2} / \mathrm{g}$ set by the Bullet Cluster [77, where $\sigma=4 \pi a_{s}^{2}$ is the self-interaction cross section. Assuming that the bound is reached (this gives a maximum bound on the mass and on the scattering length of the bosons $)$ we get $\left(a_{s} / \mathrm{fm}\right)^{2}\left(\mathrm{eV} / m c^{2}\right)=1.7710^{-8}$. From these two constraints, we obtain $m=1.6910^{-2} \mathrm{eV} / c^{2}$ and $a_{s}=1.7310^{-5} \mathrm{fm}$. This boson mass is in agreement with the limit $m<1.87 \mathrm{eV} / c^{2}$ obtained from cosmological considerations 76 .

The mass $m=2.5710^{-20} \mathrm{eV} / c^{2}$ obtained for bosons without self-interaction gives a lower bound on the mass of the bosonic dark matter particle. Inversely, the mass $m=1.6910^{-2} \mathrm{eV} / c^{2}$ obtained for self-interacting bosons in the TF limit gives an upper bound on the mass of the bosonic dark matter particle. Therefore, the typical mass of the bosonic particle lies in

considered to be at $T=0$ 137. They have a core-halo structure with a solitonic core (BEC), which is a stationary solution of the GP equation, surrounded by a halo of scalar radiation in which the density decreases as $r^{-3}$ similarly to the NavarroFrank-White (NFW) 133 and Burkert 134 profiles. This corehalo structure results from a process of gravitational cooling [24]. Dwarf dark matter halos are compact objects that have just a solitonic core (BEC) without atmosphere. Therefore, their size is equal to the size of the soliton. By contrast, large dark matter halos are extended objects with a core-halo structure. It is the radiative atmosphere that fixes the size of large dark matter halos. The atmosphere can be much larger than the size of the soliton (core). The presence of the radiative atmosphere solves the apparent paradox that BEC halos at $T=0$ should all have the same radius (in the self-interacting case) or that their radius should decrease with their mass (in the non-interacting case), in contradiction with the observations 57,58 .

19 This result assumes that (i) Willman 1 is completely condensed without radiative halo, and that (ii) the observational values of $r_{h}$ and $M_{h}$ are accurate. More precise observational data may change the value of the boson mass $m$ but its order of magnitude should remain the same. the range $2.5710^{-20} \mathrm{eV} / c^{2}<m<1.6910^{-2} \mathrm{eV} / c^{2}$. The TF limit is valid for sufficiently large scattering lengths. An estimate of the critical scattering length can be obtained by substituting $m=2.5710^{-20} \mathrm{eV} / c^{2}$ in the relation $\left(\mathrm{fm} / a_{s}\right)^{1 / 3}\left(m c^{2} / \mathrm{eV}\right)=0.654$. This gives $a_{c}=6.0710^{-59} \mathrm{fm}$. For $a_{s}<a_{c}$, the mass of the bosonic particle is $m=2.5710^{-20} \mathrm{eV} / c^{2}$ and, for $a_{c}<$ $a_{s}<1.7310^{-5} \mathrm{fm}$, the mass of the bosonic particle is $m c^{2} / \mathrm{eV}=0.654\left(a_{s} / \mathrm{fm}\right)^{1 / 3}<1.6910^{-2} \mathrm{eV} / c^{2}$.

\section{Boson stars}

General relativity is required to describe boson stars. The maximum mass of boson stars made of noninteracting bosons is $M_{\max }=0.633 \hbar \mathrm{c} / \mathrm{Gm}$ and their minimum radius is $R_{\min }=9.53 G M_{\max } / c^{2}[9]$. Introducing scaled variables, we get

$$
\frac{M_{\max }}{M_{\odot}}=8.4810^{-11} \frac{\mathrm{eV} / c^{2}}{m}, \quad \frac{R_{\min }}{\mathrm{km}}=14.1 \frac{M_{\max }}{M_{\odot}} .
$$

For $m=1 \mathrm{GeV} / c^{2}$, we obtain $M_{\max }=8.4810^{-20} M_{\odot}$ and $R_{\text {min }}=1.2010^{-18} \mathrm{~km}$. For $m=10^{-10} \mathrm{eV} / c^{2}$, we obtain $M_{\max }=0.848 M_{\odot}$ and $R_{\min }=12.0 \mathrm{~km}$. For $m=10^{-17} \mathrm{eV} / c^{2}$, we obtain $M_{\max }=8.48 \times 10^{6} M_{\odot}$ and $R_{\text {min }}=1.20 \times 10^{8} \mathrm{~km}$. For $m=2.57 \times 10^{-20} \mathrm{eV} / c^{2}$, we obtain $M_{\max }=3.30 \times 10^{9} M_{\odot}$ and $R_{\min }=1.51 \times 10^{-3} \mathrm{pc}$. Some applications of these numerical results are given in the Introduction.

The maximum mass of boson stars made of selfinteracting bosons is $M_{\max }=0.307 \hbar c^{2} \sqrt{a_{s}} /(G m)^{3 / 2}$ and their minimum radius is $R_{\min }=6.25 G M_{\max } / c^{2}$ 32 . Introducing scaled variables, we get

$$
\frac{M_{\max }}{M_{\odot}}=1.12\left(\frac{a_{s}}{\mathrm{fm}}\right)^{1 / 2}\left(\frac{\mathrm{GeV} / c^{2}}{m}\right)^{3 / 2}
$$

$$
\frac{R_{\min }}{\mathrm{km}}=9.27 \frac{M_{\max }}{M_{\odot}}
$$

We note that these results do not depend on the specific mass $m$ and scattering length $a_{s}$ of the bosons, but only on the ratio $m^{3} / a_{s}$. For $m=1 \mathrm{GeV} / c^{2}$ and $a_{s}=1 \mathrm{fm}$, we obtain $M_{\max }=1.12 M_{\odot}$ and $R_{\min }=10.4 \mathrm{~km}$. For $\left(\mathrm{fm} / a_{s}\right)^{1 / 3}\left(m c^{2} / \mathrm{eV}\right)=0.654$, we obtain $M_{\max }=6.70 \times$ $10^{13} M_{\odot}$ and $R_{\min }=20.2 \mathrm{pc}$.

\section{Appendix F: Numerical applications}

The complete equation governing the evolution of the density contrast in the matter era, Eq. 130, can be 
written in dimensionless form as

$$
\begin{aligned}
\frac{d^{2} \delta}{d a^{2}} & +\frac{3}{2 a} \frac{d \delta}{d a}+\frac{3}{2 a^{2}}\left[\frac{\kappa^{4}}{a}+\frac{\epsilon^{2} \kappa^{2}}{a^{2}}\right. \\
& \left.-\frac{1}{1+\frac{\eta^{2}}{\kappa^{2} a}}\left(1+\frac{2 \sigma}{a^{3}}\right)\left(1+\frac{3 \sigma}{a^{3}}+\frac{\nu^{2} \kappa^{2}}{a^{2}}\right)\right] \delta=0
\end{aligned}
$$

where we have defined $\kappa=k / \kappa_{Q}, \epsilon=$ $\kappa_{Q} / \kappa_{J}=\left(16 \pi \rho_{b} a^{3} a_{s}^{2} \hbar^{2} / G m^{4}\right)^{1 / 4}, \quad \eta=\kappa_{H} / \kappa_{Q}=$ $\left(4 \pi G \rho_{b} a^{3} \hbar^{2} / m^{2} c^{4}\right)^{1 / 4}$ and $\nu=\kappa_{Q} / \kappa_{C}=$ $\left(\pi G \hbar^{2} \rho_{b} a^{3} / m^{2} c^{4}\right)^{1 / 4}$. We note that $\eta=\sqrt{2} \nu$ as a consequence of Eq. 131).

It is instructive to evaluate the different quantities appearing in Eq. (F1) by specifying the mass $m$ and the scattering length $a_{s}$ of the bosons. To that purpose, we rewrite them in the convenient form

$$
\begin{aligned}
& \frac{\kappa_{Q}}{\mathrm{~m}^{-1}}=2.16 \times 10^{-10}\left(\frac{m}{\mathrm{eV} / c^{2}}\right)^{1 / 2} \\
& \frac{\kappa_{J}}{\mathrm{~m}^{-1}}=5.83 \times 10^{-18}\left(\frac{\mathrm{m}}{\mathrm{eV} / c^{2}}\right)^{3 / 2}\left(\frac{\mathrm{fm}}{a_{s}}\right)^{1 / 2} \\
& \frac{\kappa_{C}}{\mathrm{~m}^{-1}}=1.01 \times 10^{7}\left(\frac{m}{\mathrm{eV} / c^{2}}\right) \\
& \frac{\kappa_{H}}{\mathrm{~m}^{-1}}=6.49 \times 10^{-27}, \\
& \sigma=6.19 \times 10^{-19}\left(\frac{\mathrm{eV} / c^{2}}{m}\right)^{3}\left(\frac{a_{s}}{\mathrm{fm}}\right) \\
& \epsilon=3.70 \times 10^{7}\left(\frac{\mathrm{eV} / c^{2}}{m}\right)\left(\frac{a_{s}}{\mathrm{fm}}\right)^{1 / 2} \\
& \eta=\sqrt{2} \nu=3.00 \times 10^{-17}\left(\frac{\mathrm{eV} / c^{2}}{m}\right)^{1 / 2} .
\end{aligned}
$$

To obtain these results we have used the relation $\rho_{b} a^{3}=$ $\Omega_{D M, 0} \rho_{0}$, where $\Omega_{D M, 0}=0.268$ is the present fraction of dark matter and $\rho_{0}=3 H_{0}^{2} / 8 \pi G=8.42 \times 10^{-27} \mathrm{~kg} \mathrm{~m}^{-3}$ is the present density of the Universe calculated from the Hubble constant $H_{0}=67.11 \mathrm{~km} \mathrm{~s}^{-1} \mathrm{Mpc}^{-1}$ obtained from the 2014 Planck survey.

The numerical values of these parameters are given in Tables I and II for typical values of $m$ and $a_{s}$ appropriate to dark matter halos and boson stars (see Appendix E). In the matter era $\left(a>a_{i}=10^{-4}\right)$, we have

$$
\lambda_{C} \ll\left(\lambda_{Q}, \lambda_{J}\right) \ll \lambda_{H}
$$

Indeed, $\quad \lambda_{C} / \lambda_{Q}=\kappa_{Q} / \kappa_{C} a^{3 / 4}=\nu / a^{3 / 4}<10^{-3}$, $\lambda_{C} / \lambda_{J}=\kappa_{J} / \kappa_{C}=\nu / \epsilon<10^{-3}, \lambda_{Q} / \lambda_{H}=\kappa_{H} / \kappa_{Q} a^{3 / 4}=$ $\eta / a^{3 / 4}<10^{-3}, \lambda_{J} / \lambda_{H}=\kappa_{H} / \kappa_{J} a^{3 / 2}=\eta \epsilon / a^{3 / 2}<10^{-3}$, and $\lambda_{C} / \lambda_{H}=\kappa_{H} / \kappa_{C} a^{3 / 2}=\eta \nu / a^{3 / 2}<10^{-6}$. The Tables make clear that relativistic effects are very weak in the matter era (except for perturbations with very large wavelengths) in agreement with the discussion given in the paper.

\section{Appendix G: The smallness of the speed of sound in the matter era}

The speed of sound is defined by $c_{s}^{2}=P^{\prime}(\epsilon) c^{2}$, where $\epsilon$ is the energy density. In the matter era, $\epsilon \sim \rho c^{2}$, where $\rho$ is the rest-mass density, so that $c_{s}^{2}=P^{\prime}(\rho)$. For a selfinteracting $\mathrm{SF}$, the speed of sound is given by Eq. (59). It may be considered as obvious that $c_{s} \ll c$ during the matter era. Actually, there is a nice way to prove this statement. Using $\rho_{b} a^{3}=\Omega_{D M, 0} \rho_{0}$, and Eq. 122 , we obtain

$$
\frac{c_{s}^{2}}{c^{2}}=\frac{3 a_{s} \hbar^{2} \Omega_{D M, 0} H_{0}^{2}}{2 G m^{3} a^{3} c^{2}} .
$$

This quantity depends on the mass $m$ and scattering length $a_{s}$ of the bosons only through the ratio $a_{s} / \mathrm{m}^{3}$. It turns out that this ratio also determines the size of dwarf dark matter halos through the relation (see [57, 64, 66, 67] and Appendix E):

$$
R=\pi\left(\frac{a_{s} \hbar^{2}}{G m^{3}}\right)^{1 / 2}
$$

As a result, Eq. G1 can be rewritten as

$$
\frac{c_{s}}{c}=\left(\frac{3 \Omega_{D M, 0}}{2 \pi^{2}}\right)^{1 / 2} \frac{H_{0} R}{a^{3 / 2} c} .
$$

Since $a>a_{i}=10^{-4}$ in the matter era, and since the size of the DM halos is obviously much smaller than the present horizon $\left(R \ll c / H_{0}\right)$, we conclude that $c_{s} \ll c$ during the matter era. We have reached this conclusion without having to specify the mass and scattering length of the bosons. We can explicitly check on Table II that the condition $\sigma / a^{3} \ll 1$, equivalent to $c_{s} \ll c$, is indeed satisfied for the typical values of the mass $m$ and scattering length $a_{s}$ of the bosons considered in the literature.

\section{Acknowledgments}

A. S. acknowledges CONACyT for the postdoctoral grant received. 


\begin{tabular}{|c|c|c|c|c|}
\hline Physical system & $\kappa_{Q}\left(\mathrm{~m}^{-1}\right)$ & $\kappa_{J}\left(\mathrm{~m}^{-1}\right)$ & $\kappa_{C}\left(\mathrm{~m}^{-1}\right)$ & $\kappa_{H}\left(\mathrm{~m}^{-1}\right)$ \\
\hline DM halos & & & & \\
\hline$a_{s}=0, m=2.57 \times 10^{-20} \mathrm{eV} / \mathrm{c}^{2}$ & $3.46 \times 10^{-20}$ & $+\infty$ & $2.60 \times 10^{-13}$ & $6.49 \times 10^{-27}$ \\
\hline$a_{s}=1.73 \times 10^{-5} \mathrm{fm}, \mathrm{m}=1.69 \times 10^{-2} \mathrm{eV} / \mathrm{c}^{2}$ & $2.81 \times 10^{-11}$ & $3.08 \times 10^{-18}$ & $1.71 \times 10^{5}$ & $6.49 \times 10^{-27}$ \\
\hline$a_{s}=6.07 \times 10^{-59} \mathrm{fm}, \mathrm{m}=2.57 \times 10^{-20} \mathrm{eV} / \mathrm{c}^{2}$ & $3.46 \times 10^{-20}$ & $3.08 \times 10^{-18}$ & $2.60 \times 10^{-13}$ & $6.49 \times 10^{-27}$ \\
\hline Boson stars & & & & \\
\hline$a_{s}=0, m=1 \times 10^{9} \mathrm{eV} / \mathrm{c}^{2}$ & $6.83 \times 10^{-6}$ & $+\infty$ & $1.01 \times 10^{16}$ & $6.49 \times 10^{-27}$ \\
\hline$a_{s}=0, m=1 \times 10^{-10} \mathrm{eV} / \mathrm{c}^{2}$ & $2.16 \times 10^{-15}$ & $+\infty$ & $1.01 \times 10^{-3}$ & $6.49 \times 10^{-27}$ \\
\hline$a_{s}=0, m=1 \times 10^{-17} \mathrm{eV} / \mathrm{c}^{2}$ & $6.83 \times 10^{-19}$ & $+\infty$ & $1.01 \times 10^{-10}$ & $6.49 \times 10^{-27}$ \\
\hline$a_{s}=1 \mathrm{fm}, \mathrm{m}=1 \times 10^{9} \mathrm{eV} / \mathrm{c}^{2}$ & $6.83 \times 10^{-6}$ & $1.84 \times 10^{-4}$ & $1.01 \times 10^{16}$ & $6.49 \times 10^{-27}$ \\
\hline
\end{tabular}

TABLE I: Values of the parameters that appear in the equation for the density contrast for different astrophysical systems.

\begin{tabular}{|c|c|c|c|c|}
\hline Physical system & $\epsilon$ & $\sigma$ & $\eta$ & $\nu$ \\
\hline DM halos & & & & \\
\hline$a_{s}=0, m=2.57 \times 10^{-20} \mathrm{eV} / \mathrm{c}^{2}$ & 0 & 0 & $1.87 \times 10^{-7}$ & $1.33 \times 10^{-7}$ \\
\hline$a_{s}=1.73 \times 10^{-5} \mathrm{fm}, \mathrm{m}=1.69 \times 10^{-2} \mathrm{eV} / \mathrm{c}^{2}$ & $9.11 \times 10^{6}$ & $2.22 \times 10^{-18}$ & $2.31 \times 10^{-16}$ & $1.65 \times 10^{-16}$ \\
\hline$a_{s}=6.07 \times 10^{-59} \mathrm{fm}, \mathrm{m}=2.57 \times 10^{-20} \mathrm{eV} / \mathrm{c}^{2}$ & $1.12 \times 10^{-2}$ & $2.21 \times 10^{-18}$ & $1.87 \times 10^{-7}$ & $1.33 \times 10^{-7}$ \\
\hline Boson stars & & & & \\
\hline$a_{s}=0, m=1 \times 10^{9} \mathrm{eV} / \mathrm{c}^{2}$ & 0 & 0 & $9.49 \times 10^{-22}$ & $6.77 \times 10^{-22}$ \\
\hline$a_{s}=0, m=1 \times 10^{-10} \mathrm{eV} / \mathrm{c}^{2}$ & 0 & 0 & $3.00 \times 10^{-12}$ & $2.14 \times 10^{-12}$ \\
\hline$a_{s}=0, m=1 \times 10^{-17} \mathrm{eV} / \mathrm{c}^{2}$ & 0 & 0 & $9.49 \times 10^{-9}$ & $6.77 \times 10^{-9}$ \\
\hline$a_{s}=1 \mathrm{fm}, m=1 \times 10^{9} \mathrm{eV} / \mathrm{c}^{2}$ & $3.70 \times 10^{-2}$ & $6.19 \times 10^{-46}$ & $9.49 \times 10^{-22}$ & $6.77 \times 10^{-22}$ \\
\hline
\end{tabular}

TABLE II: Values of the parameters that appear in the equation for the density contrast for different astrophysical systems.

[1] E. W Kolb, M. S. Turner, The Early Universe (AddisonWesley Publishing Company, 1989)

[2] A. Zee, Quantum Field Theory in a Nutshell (Princeton University Press, 2003)

[3] S. Dodelson, Modern Cosmology (Academic Press, 2003)

[4] O. Klein, Z. Phys. 37, 895 (1926)

[5] W. Gordon, Z. Phys. 40, 117 (1926)

[6] O. Klein, Z. Phys. 41, 407 (1927)

[7] E. Schrödinger, Ann. Phys. (Berlin) 386, 109 (1926)

[8] E. Schrödinger, Phys. Rev. 28, 1049 (1926)

[9] D.J. Kaup, Phys. Rev. 172, 1331 (1968)

[10] R. Ruffini, S. Bonazzola, Phys. Rev. 187, 1767 (1969)

[11] W. Thirring, Phys. Lett. B 127, 27 (1983)

[12] J.D. Breit, S. Gupta, A. Zaks, Phys. Lett. B 140, 329 (1984)

[13] E. Takasugi, M. Yoshimura, Z. Phys. C 26, 241 (1984)

[14] M. Colpi, S.L. Shapiro, I. Wasserman, Phys. Rev. Lett. 57, 2485 (1986)

[15] J.J. van der Bij, M. Gleiser, Phys. Lett. B 194, 482 (1987)

[16] M. Gleiser, Phys. Rev. D 38, 2376 (1988)

[17] R. Ferrell, M. Gleiser, Phys. Rev. D 40, 2524 (1989)

[18] M. Gleiser, R. Watkins, Nucl. Phys. B 319, 733 (1989)

[19] E. Seidel, W.M. Suen, Phys. Rev. D 42, 384 (1990)

[20] F.V. Kusmartsev, E.W. Mielke, F.E. Schunck, Phys. Lett. A 157, 465 (1991)

[21] F.V. Kusmartsev, E.W. Mielke, F.E. Schunck, Phys. Rev. D 43, 3895 (1991)
[22] T.D. Lee, Y. Pang, Phys. Rep. 221, 251 (1992)

[23] P. Jetzer, Phys. Rep. 220, 163 (1992)

[24] E. Seidel, W.M. Suen, Phys. Rev. Lett. 72, 2516 (1994)

[25] J. Balakrishna, E. Seidel, W.M. Suen, Phys. Rev. D 58, 104004 (1998)

[26] F.E. Schunck, A.R. Liddle, Black Holes: Theory and Observation, Proceedings of the 179th W. E. Heraeus Seminar. Edited by Friedrich W. Hehl, Claus Kiefer, Ralph J. K. Metzler. Springer (1998), P. 285

[27] E.W. Mielke, F.E. Schunck, Nuc. Phys. B 564, 185 (2000)

[28] D.F. Torres, S. Capozziello, G. Lambiase, Phys. Rev. D 62, 104012 (2000)

[29] X.Z. Wang, Phys. Rev. D 64, 124009 (2001)

[30] F.E. Schunck, E.W. Mielke, Class. Quantum Grav. 20, R301 (2003)

[31] F.S. Guzmán, Phys. Rev. D 73, 021501 (2006)

[32] P.H. Chavanis, T. Harko, Phys. Rev. D 86, 064011 (2012)

[33] E. Seidel, W.-M. Suen, Phys. Rev. Lett. 66, 1659 (1991)

[34] L.A. Ureña-López, Class. Quantum Grav. 19, 2617 (2002)

[35] A. Suárez, V.H. Robles, T. Matos, Astrophys. Space Sci. Proc. 38, 107 (2014)

[36] T. Rindler-Daller, P.R. Shapiro, Astrophys. Space Sci. Proc. 38, 163 (2014)

[37] P.H. Chavanis, Self-gravitating Bose-Einstein condensates, in Quantum Aspects of Black Holes, edited by X. 
Calmet (Springer, 2015)

[38] M.R. Baldeschi, G.B. Gelmini, R. Ruffini, Phys. Lett. B 122, 221 (1983)

[39] M. Membrado, A.F. Pacheco, J. Sanudo, Phys. Rev. A 39, 4207 (1989)

[40] S.J. Sin, Phys. Rev. D 50, 3650 (1994)

[41] S.U. Ji, S.J. Sin, Phys. Rev. D 50, 3655 (1994)

[42] F.E. Schunck, astro-ph/9802258

[43] T. Matos, F.S. Guzmán, F. Astron. Nachr. 320, 97 (1999)

[44] F.S. Guzmán, T. Matos, Class. Quantum Grav. 17, L9 (2000)

[45] W. Hu, R. Barkana, A. Gruzinov, Phys. Rev. Lett. 85, $1158(2000)$

[46] T. Matos, L.A. Ureña-López, Phys. Rev. D 63, 063506 (2001)

[47] A. Arbey, J. Lesgourgues, P. Salati, Phys. Rev. D 64, $123528(2001)$

[48] M.P. Silverman, R.L. Mallett, Class. Quantum Grav. 18, L103 (2001)

[49] M. Alcubierre, F.S. Guzmán, T. Matos, D. Núñez, L.A. Ureña-López, P. Wiederhold, Class. Quantum. Grav. 19, $5017(2002)$

[50] M.P. Silverman, R.L. Mallett, Gen. Rel. Grav. 34, 633 (2002)

[51] A. Bernal, T. Matos, D. Núñez, Rev. Mex. Astron. Astrofis. 44, 149 (2008)

[52] P. Sikivie, Q. Yang, Phys. Rev. Lett. 103, 111301 (2009)

[53] T. Matos, A. Vázquez-González, J. Magaña, Mon. Not. R. Astron. Soc. 393, 1359 (2009)

[54] J.W. Lee, Phys. Lett. B 681, 118 (2009)

[55] T.P. Woo, T. Chiueh, Astrophys. J. 697, 850 (2009)

[56] J.W. Lee, S. Lim, J. Cosmol. Astropart. Phys. 01, 007 (2010)

[57] P.H. Chavanis, Phys. Rev. D 84, 043531 (2011)

[58] P.H. Chavanis, L. Delfini, Phys. Rev. D 84, 043532 (2011)

[59] G. Manfredi, P.A. Hervieux, F. Haas, Class. Quantum Grav. 30, 075006 (2013)

[60] H.Y. Schive, T. Chiueh, T. Broadhurst, Nature Physics 10, 496 (2014)

[61] H.Y. Schive et al., Phys. Rev. Lett. 113, 261302 (2014)

[62] J.W. Lee, I. Koh, Phys. Rev. D 53, 2236 (1996)

[63] P.J.E. Peebles, Astrophys. J. 534, L127 (2000)

[64] J. Goodman, New Astronomy 5, 103 (2000)

[65] J. Lesgourgues, A. Arbey, P. Salati, New Astron. Rev. 46, $791(2002)$

[66] A. Arbey, J. Lesgourgues, P. Salati, Phys. Rev. D 68, $023511(2003)$

[67] C.G. Böhmer, T. Harko, J. Cosmol. Astropart. Phys. 06, 025 (2007)

[68] F. Briscese, Phys. Lett. B 696, 315 (2011)

[69] T. Harko, J. Cosmol. Astropart. Phys. 05, 022 (2011)

[70] M.O.C. Pires, J.C.C. de Souza, J. Cosmol. Astropart. Phys. 11 (2012) 024

[71] V.H. Robles, T. Matos, Monthly Not. Roy. Astron. Soc. 422, 282 (2012)

[72] T. Rindler-Daller, P. R. Shapiro, Monthly Not. Roy. Astron. Soc. 422, 135 (2012)

[73] V. Lora, J. Magaña, A. Bernal, F.J. Sánchez-Salcedo, E.K. Grebel, J. Cosmol. Astropart. Phys. 02, 011 (2012)

[74] A.X. González-Morales, A. Diez-Tejedor, L.A. UreñaLópez, O. Valenzuela, Phys. Rev. D 87, 021301(R) (2013)
[75] F.S. Guzmán, F.D. Lora-Clavijo, J.J. González-Avilés, F.J. Rivera-Paleo, J. Cosmol. Astropart. Phys. 09 (2013) 034

[76] T. Fukuyama, M. Morikawa, T. Tatekawa, J. Cosmol. Astropart. Phys. 06 (2008) 033

[77] S.W. Randall, M. Markevitch, D. Clowe, A.H. Gonzalez, M. Bradac, Astrophys. J. 679, 1173 (2008)

[78] A. A. Starobinsky, Phys. Lett. B 91, 99 (1980)

[79] A.H. Guth, Phys. Rev. D 23, 347 (1981)

[80] A.D. Linde, Phys. Lett. B 108, 389 (1982)

[81] A. Albrecht, P.J. Steinhardt, M.S. Turner, F. Wilczek, Phys. Rev. Lett. 48, 1437 (1982)

[82] A. Linde, Particle Physics and Inflationary Cosmology (Harwood, Chur, Switzerland, 1990)

[83] K. A. Olive, Phys. Rep. 190, 308 (1990)

[84] B. Ratra, J. Peebles, Phys. Rev. D 37, 321 (1988)

[85] A.A. Starobinski, JETP Lett. 68, 757 (1998)

[86] R.R. Caldwell, R. Dave, P.J. Steinhardt, Phys. Rev. Lett. 80, 1582 (1998)

[87] Ph. Brax, J. Martin, Phys. Lett. B 468, 40 (1999)

[88] A. Albrecht, C. Skordis, Phys. Rev. Lett. 84, 2076 (2000)

[89] T. Barreiro, E.J. Copeland, N.J. Nunes, Phys. Rev. D 61, $127301(2000)$

[90] L.A. Ureña-López, T. Matos, Phys. Rev. D 62, 081302(R) (2000)

[91] P. Brax, J. Martin, Phys. Rev. D 61, 103502 (2000)

[92] T.D. Saini, S. Raychaudhury, V. Sahni, A.A. Starobinsky, Phys. Rev. Lett. 85, 1162 (2000)

[93] V. Sahni, A.A. Starobinsky, Int. J. Mod. Phys. D 9, 373 (2000)

[94] V. Sahni, Class. Quantum Grav. 19, 3435 (2002)

[95] M. Pavlov, C. Rubano, M. Sazhin, P. Scudellaro, Astrophys. J. 566, 619 (2002)

[96] V. Sahni, T.D. Saini, A.A. Starobinsky, U. Alam, JETP Lett. 77, 201 (2003)

[97] A. Suárez, T. Matos, Mon. Not. R. Astron. Soc. 416 , 87 (2011)

[98] J. Magaña, T. Matos, A. Suárez, F. J. Sánchez-Salcedo, JCAP 10, 003 (2012)

[99] M.Yu. Khlopov, B.A. Malomed, Ya.B. Zeldovich, Mon. Not. R. astr. Soc. 215, 575 (1985)

[100] P.H. Chavanis, Astron. Astrophys. 537, A127 (2012)

[101] T. Harko, Mon. Not. R. Astron. Soc. 413, 3095 (2011)

[102] F. Dalfovo, S. Giorgini, L.P. Pitaevskii, S. Stringari, Rev. Mod. Phys. 71, 463 (1999)

[103] P.H. Chavanis, arXiv:1412.0005

[104] B. Li, T. Rindler-Daller, P.R. Shapiro, Phys. Rev. D 89 , 083536 (2014)

[105] E. Madelung, Zeit. F. Phys. 40, 322 (1927)

[106] D. Bohm, Phys. Rev. 85, 166 (1952)

[107] T. Takabayasi, Prog. Theor. Phys. 8, 143 (1952)

[108] T. Takabayasi, Prog. Theor. Phys. 9, 187 (1953)

[109] L. Onsager, Nuovo Cimento 6, 279 (1949)

[110] R.P. Feynman, Progress in Low Temperature Physics, Vol. 1. (North-Holland, Amsterdam, 1955)

[111] A. Suárez, T. Matos, Class. Quantum Grav. 31, 045015 (2014)

[112] A. Suárez, P.H. Chavanis, arXiv:1504.01164

[113] S. Weinberg, Gravitation and Cosmology (John Wiley, 2002)

[114] C.-P. Ma, E. Bertschinger, Astrophys. J. 455, 7 (1995)

[115] V. F. Mukhanov, H. A. Feldman, R. H. Brandenberger, Phys. Rep. 215, 203 (1992) 
[116] J.H. Jeans, Astronomy and Cosmogony (Cambridge University Press, 1929)

[117] J. Binney, S. Tremaine, Galactic Dynamics (Princeton Series in Astrophysics, 1987)

[118] J.-A. Gu, W.-Y.P. Hwang, Phys. Lett. B 517, 1 (2001)

[119] M.D. Lehnert et al., Nature 467, 940 (2010)

[120] C. Clarkson, G. Ellis, J. Larena et al., Rept. Prog. Phys. 74, 112901 (2011)

[121] G. Dvali, C. Gomez, Fortschr. Phys. 61, 742 (2013)

[122] R. Casadio, A. Orlandi, J. High Energy Phys. 8, 25 (2013)

[123] W. G. Unruh, Phys. Rev. Lett. 46, 1351 (1981)

[124] G. E. Volovik, Grav. Cosmol. Suppl. 6, 187 (2000)

[125] M. Novello, S. Perez Bergliaffa, J. Salim, V.A. DeLorenci, R. Klippert, Class. Quant. Grav. 20, 859 (2003)

[126] L. J. Garay, J. R. Anglin, J. I. Cirac, P. Zoller, Phys. Rev. Lett. 85, 4643 (2000).

[127] C. Barcelo, S. Liberati, M. Visser, Class. Quantum. Grav. 18, 1137 (2001)
[128] P. O. Fedichev, U. R. Fischer, Phys. Rev. Lett. 91, 240407 (2003)

[129] C. Barcelo, S. Liberati, M. Visser, Phys. Rev. A 68, 053613 (2003)

[130] L.M. Widrow, N. Kaiser, Astrophys. J. Lett. 416, L71 (1993)

[131] P.H. Chavanis, Phys. Rev. D 84, 063518 (2011)

[132] G. Kauffmann, S.D.M. White, B. Guiderdoni, Mon. Not. R. astr. Soc. 264, 201 (1993)

[133] J.F. Navarro, C.S. Frenk, S.D.M. White, Astrophys. J. 462, 563 (1996)

[134] A. Burkert, Astrophys. J. 447, L25 (1995)

[135] C. Destri, H.J. de Vega, N.G. Sanchez, Astroparticle Physics 46, 14 (2013)

[136] H.J. de Vega, P. Salucci, N.G. Sanchez, Mon. Not. R. Astron. Soc. 442, 2717 (2014)

[137] P.H. Chavanis, M. Lemou, F. Méhats, arXiv:1409.7840 